\title{
Falling liquid films in narrow tubes: occlusion scenarios
}

\author{
Georg F. Dietze ${ }^{1} \dagger$, G. Lavalle ${ }^{2}$ and C. Ruyer-Quil ${ }^{3}$ \\ ${ }^{1}$ Université Paris-Saclay, CNRS, FAST, 91405, Orsay, France. \\ ${ }^{2}$ Université Paris-Saclay, CNRS, LIMSI, 91405, Orsay, France. \\ ${ }^{3}$ Université Savoie Mont Blanc
}

(Received 26 February 2020; revised xx; accepted xx)

\begin{abstract}
We study a gravity-driven wavy liquid film falling down the inner surface of a narrow cylindrical tube in the presence of an active core gas flow. We employ the model of Dietze and Ruyer-Quil (J. Fluid Mech., vol. 762, 2015, pp. 68-109) to investigate the role of surface waves in the occlusion of the tube. We consider four real working liquids and reproduce several experiments from the literature, focusing on conditions where the Bond number is greater or equal to unity. We prove that occlusion is triggered by spatially growing surface waves beyond the limit of saturated travelling-wave solutions, and delimit three possible regimes for a naturally evolving wavy film: (i) certain occlusion, when the liquid Reynolds number is greater than the limit of the spatially most-amplified travelling waves. Occlusion is caused by surface waves emerging from linear wave selection (scenario I); (ii) conditional occlusion, when the most-amplified waves possess travelling states but longer waves don't. Occlusion is triggered by secondary instability, generating long waves through nonlinear coarsening dynamics (scenario II); and (iii) impossible occlusion, when travelling waves always exist, no matter how great their wavelength. We show that certain occlusion is delayed by gravity and precipitated by a counter-current gas flow, axial viscous diffusion (high-viscosity liquids), and inertia (low-viscosity liquids). The latter two effects are also found to determine whether the occlusion mechanism is dictated by loss of travelling-wave solutions or absolute instability. Finally, we show that occlusion can be prevented through coherent inlet forcing. As a side benefit, we introduce an augmented version of our model based on a localized additional force term that allows representing stable travelling liquid pseudo-plugs.
\end{abstract}

Key words: Thin films

\section{Introduction}

We consider the configuration in figure 1, a thin liquid film falling down the inner surface of a vertical cylindrical tube of radius $R^{\star}$ under the action of the gravitational acceleration $\mathrm{g}$. The film of liquid (denoted with the subscript l) is in contact with a laminar flow of gas in the core (subscript g). Both fluids are assumed to be Newtonian with constant densities $\rho_{\mathrm{l}}$ and $\rho_{\mathrm{g}}$, dynamic viscosities $\mu_{\mathrm{l}}$ and $\mu_{\mathrm{g}}$, and surface tension $\sigma$. Also, we assume the arrangement to be axisymmetric. We denote the film thickness $h$, the core radius $d$, its spatial average $\bar{d}$, and the flow rates $q_{1}$ and $q_{\mathrm{g}}$. Their dimensional

$\dagger$ Email address for correspondence: dietze@fast.u-psud.fr 
(a)

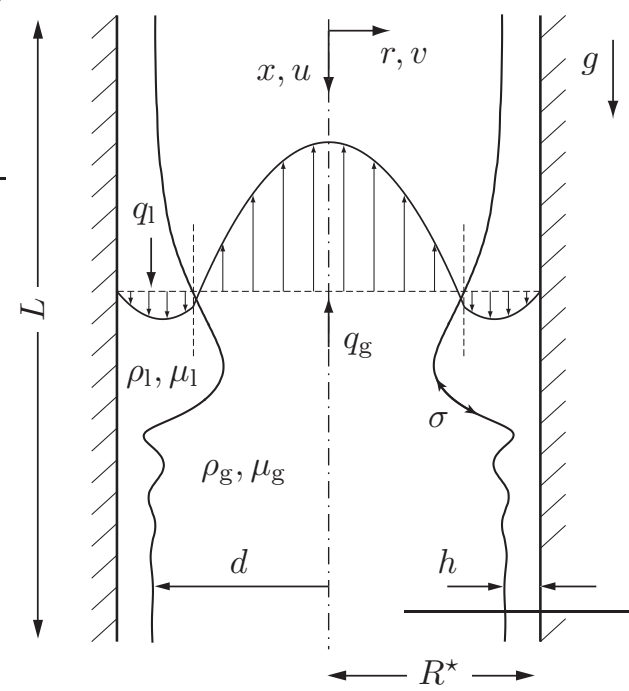

(b)

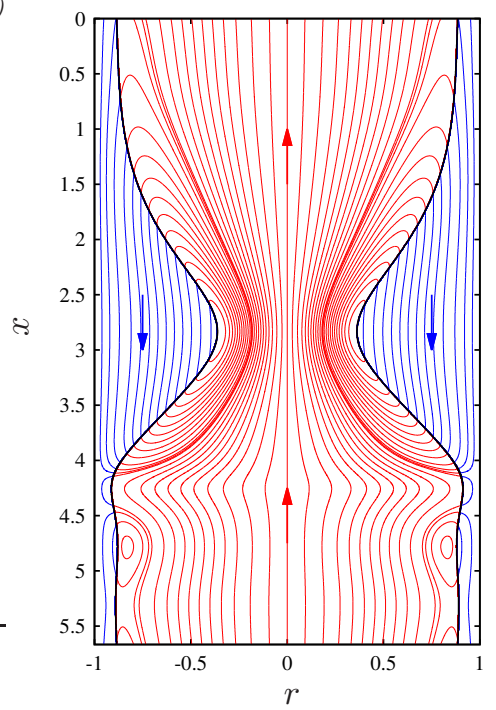

FiguRE 1. Falling liquid film (subscript 1) lining the inner surface of a narrow cylindrical tube in contact with a laminar gas flow in the core (subscript g). (a) Problem configuration and notations. The tube radius $R^{\star}$ is the length scale, the star superscript designating dimensional quantities; (b) example of a travelling-wave computation with our model (2.5): case 4 in table 1 (low-viscosity silicone oil), $R e=14.6, R_{g}=-17.27$ (limit point marked by asterisk in panel 13a). Streamlines in the wall-fixed reference frame within the liquid (blue lines) and gas (red lines).

counterparts will be distinguished by a star, e.g. $d^{\star}$, and their counterparts in the corresponding flat-film primary flow will be denoted with a subscript zero, e.g. $d_{0}$.

We focus on conditions, where: (i) the effect of gravity, quantified through the Bond number $\mathrm{Bo}=\rho_{1} g R^{\star 2} / \sigma$, is at least comparable to that of capillarity (Bo $\geqslant 1$ ); (ii) the liquid film's inertia, quantified through the Reynolds number $\operatorname{Re}=q_{10}^{\star} \rho_{1} /\left(\pi R^{\star} \mu_{1}\right)$, is not necessarily negligible $(\operatorname{Re} \leqslant 20)$; and (iii) the effect of the gas flow can be relevant, but its Reynolds number $\operatorname{Re}_{\mathrm{g}}=q_{\mathrm{g} 0}^{\star} \rho_{\mathrm{g}} /\left(\pi R^{\star} \mu_{\mathrm{g}}\right)$ remains moderate $\left(\left|\operatorname{Re}_{\mathrm{g}}\right|<20\right)$.

We investigate this flow with the weighted residual integral boundary layer (WRIBL) model of Dietze \& Ruyer-Quil (2015), which was previously applied only to gravity-free films. The model accounts for gravity, inertia, full interfacial curvature, axial viscous diffusion, and full inter-phase coupling. Our model computations are confronted with our own direct numerical simulations (DNS) in appendix A and panel 5d.

We focus on the occlusion of the narrow tube due to the interfacial instability of the falling liquid film, as observed experimentally by Dao \& Balakotaiah (2000) and Camassa et al. (2014). Occlusion is relevant for chemical engineering applications such as tubular falling film micro-reactors (Seebauer et al. 2012). It also occurs in the human pulmonary airways due to the collapse of the mucus-serous film lining their inner surface (Grotberg 2011). In the lower generations of bronchioles, the effect of gravity and possibly inertia is not necessarily negligible (Kamm \& Schroter 1989).

The falling liquid film is subject to two instability mechanisms causing interfacial deformations: the Plateau-Rayleigh mechanism (Plateau 1849; Rayleigh 1892; Goren 1962), due to the azimuthal curvature of the film surface; and the Kapitza mechanism (Kapitza 1948), due to inertia, which sets in as a result of the gravity-driven mean flow (Brooke Benjamin 1957; Yih 1963). The strength of this mean flow determines whether 
the absolute nature of the Plateau-Rayleigh instability or the convective nature of the Kapitza instability dominates (Duprat et al. 2007). For the liquids studied here, we find that occlusion is dictated by absolute instability when Bo $\ll 1$, whereas convective instability is responsible for occlusion when $\mathrm{Bo} \geqslant 1$.

In the case of convective instability, the liquid film can produce travelling surface waves, which remain stationary in their reference frame. Gravity plays an important role in shaping these waves, compressing the leading and elongating the trailing wave front, which results in asymmetric tear-shaped wave humps (Dietze 2016). The resulting distortion of the film surface favours variations in axial (stabilizing) over azimuthal (destabilizing) surface curvature. In related systems, this mechanism has been found to saturate the Plateau-Rayleigh instability in the weakly-nonlinear regime (Frenkel et al. 1987), to the extent that the film looks stable to the naked eye (Quéré 1990). In our current problem, we find that it greatly extends the region of existence of stronglynonlinear travelling waves and considerably delays occlusion.

Trifonov (1992) applied the integral boundary layer approach of Shkadov (1967) to model an annular liquid film falling in a vertical cylindrical tube. The author found that the Plateau-Rayleigh mechanism systematically increases the amplitude of travelling waves versus a planar falling liquid film, where only the Kapitza mechanism is active. He also observed catastrophic growth, when the amplitude of travelling-wave solutions diverged as a function of $1 / R$. In those instances, the effect of gravity was negligible, i.e. Bo $\ll 1$. By contrast, we focus on occlusion in the limit Bo $\geqslant 1$. Moreover, the model of Trifonov (1992) did not account for axial viscous diffusion, which we find to greatly precipitate occlusion in high-viscosity films.

Dao \& Balakotaiah (2000) performed falling-film experiments in long narrow tubes and determined the occlusion onset in terms of Re for a wide range of liquids. However, no information was available on the wave dynamics responsible for occlusion. By reproducing two of these experiments (runs 13 and 20 there) with our own spatio-temporal computations, we provide this missing information, leading us to distinguish two wave-induced occlusion scenarios. In scenario I, waves causing occlusion emerge directly from linear wave selection. In scenario II, they result from the secondary instability of a regular train of travelling waves, triggering a cascade of coalescence/absorption events that produce increasingly long, more dangerous, waves. The secondary instability (Liu \& Gollub 1993) and the ensuing cascaded coarsening dynamics (Chang et al. 1996b) are well known phenomena in planar falling liquid films.

Jensen (2000) investigated annular collars travelling on a gravity-driven liquid film lining the inner surface of a cylindrical tube. The collars were constructed from unduloids, i.e. symmetric constant-curvature equilibrium shapes (Delaunay 1841). The author studied under what conditions collars may grow to occlude the tube. Our study extends this work in that the collar shape is not forced to be symmetric, but follows implicitly from the evolution equations. This allows for the gravity-induced distortion of collars, which we find can greatly delay occlusion.

Camassa et al. (2014) performed falling-film occlusion experiments in a transparent tube, using a high-viscosity silicone oil. By confronting these with linear stability calculations, the authors concluded that occlusion is caused by spatially growing nonlinear waves and not by absolute linear instability $(\mathrm{Bo}=11.06$ in their experiments). Further, with the help of a first-order long-wave model obtained from asymptotic expansion (Benney 1966), the authors constructed travelling-wave solutions. By continuing these at fixed wave length in terms of Re, they identified a limit point (LP), and conjectured that this may signal the occlusion onset in a real system. However, the authors cautioned that further work was needed to verify this w.r.t. their experiments. This work was initiated 
by Camassa et al. (2016), but their model did not account for axial viscous diffusion, which we show to strongly affect travelling wave solutions, and the wavelength assumed in their continuation was too long compared to the experiment.

In our current study, we have checked the conjecture of Camassa et al. (2014) based on travelling-wave and spatio-temporal computations with our model. We have successfully confronted these computations with the experiments of Camassa et al. (2014) and find that accounting for axial viscous diffusion greatly improves agreement. Based on our computations, we find that the surface waves causing occlusion indeed systematically lie beyond the limit of travelling-wave solutions (figure 4), confirming the conjecture of Camassa et al. (2014). This limit is highly sensitive to the considered wavelength, long waves being more dangerous than short ones, as shown by Camassa et al. (2016) and Ding et al. (2019). Thus, any predictive criterion for occlusion based on travelling-wave solutions must account for the type of waves that actually occur in a real system.

For this, we introduce the upper conservative occlusion bound $\mathrm{Re}_{\max }$, which corresponds to the limit point of travelling-wave solutions at the spatially most amplified frequency of linear waves $f_{\max }$. For $R e>R_{\max }$, the most amplified surface waves, which typically emerge in an experiment, do not possess travelling states and occlusion is certain to occur in a naturally evolving film. We find that the occlusion experiments of Camassa et al. (2014) and experimental run 20 in Dao \& Balakotaiah (2000) correspond to this regime. Occlusion in this case occurs through scenario $I$. To determine $\mathrm{Re}_{\max }$, we have computed the most amplified wave frequency $f_{\max }$ in our numerical continuations by solving the linear spatial stability problem along with our nonlinear model equations. This had not been attempted by Camassa et al. (2014) and Ding et al. (2019).

At the other end, occlusion is delimited by a lower conservative bound $R e=R_{0}$, below which travelling wave solutions always exist, no matter how great the wavelength, and occlusion is impossible. The existence of such a limit was discovered by Ding et al. (2019).

We find that the conservative occlusion bounds $\mathrm{Re}_{0}$ and $\mathrm{Re}_{\max }$ delimit a region of conditional occlusion, $\operatorname{Re}_{0}<\operatorname{Re}<\operatorname{Re}_{\max }$, where occlusion is theoretically possible (for long enough waves) but does not necessarily occur in a real system. Whether it does, depends on the specific wave dynamics that unfolds over the spatio-temporal evolution of the film, and whether the tube is long enough to accommodate this. In this regime, we find that occlusion is caused by scenario II and that it applies to experimental run 13 in Dao \& Balakotaiah (2000). It also opens the possibility of preventing occlusion through coherent inlet forcing, i.e. by forcing waves of sufficiently high frequency. We validate this idea based on spatio-temporal computations.

Unless Bo is very large, we find that the height of travelling waves at the limit point $\mathrm{Re}=\mathrm{Re}_{\max }$ is far from reaching the tube radius. In contrast to what Dao \& Balakotaiah (2000) conjectured, travelling-wave solutions are thus lost abruptly and not due to the wave height reaching the tube radius continuously.

Zhou et al. (2016) investigated viscoelastic liquid films falling in a deformable narrow tube. The authors performed calculations with a model based on the same approach as Trifonov (1992), which does not account for axial viscous diffusion. Interestingly, it was shown that occlusion can cause a contraction of the flexible tube. Such events are known to occur in the pulmonary airways (Grotberg 2011) and can damage cells within the capillaries (Bian et al. 2010). The authors went on to determine the occlusion onset by varying the liquid flow rate in spatio-temporal computations. This onset was defined as the point at which the computation breaks down due to an occlusion event. However, such computations cannot attain a representative developed state. For example, when starting from a flat-film initial condition, a single unrealistically large tsunami-like wave usually develops in the early stages. This wave is bound to cause occlusion, but 
it is not representative of a real system. By contrast, we characterize occlusion based on the bounds $\mathrm{Re}_{0}$ and $\mathrm{Re}_{\max }$ of travelling-wave solutions, and our spatio-temporal computations have all been continued until reaching a statistically-developed state. To achieve this, we have allowed our computations to continue past occlusion events by numerically limiting the core radius of an occluded region to a small but finite value.

Liu \& Ding (2017) studied a vertically falling glycerol film flowing down a porous cylindrical surface using a lubrication equation. The authors found that porosity precipitates both the absolute instability threshold and the occlusion limit. Using the same approach, Ding et al. (2019) studied the effect of the Marangoni instability on a radiallyheated liquid film falling in a vertical cylindrical tube. The authors found that Marangoni stresses promote/delay occlusion when the film is heated/cooled from the wall. However, their modelling approach does not account for inertia nor axial viscous diffusion. We find that this does not allow to accurately predict occlusion in high-viscosity (because of axial viscous diffusion) or low-viscosity (because of inertia) liquids.

The model employed in our current study extends upon the earlier works of Trifonov (1992), Camassa et al. (2014, 2017), Zhou et al. (2016), Liu \& Ding (2017), and Ding et al. (2019) by accounting for axial viscous diffusion and inertia. This has allowed us to investigate four real liquids, for which we determine the lower and upper occlusion bounds $\mathrm{Re}_{0}$ and $\mathrm{Re}_{\max }$ and characterize the possible occlusion scenarios. When the viscosity is high, we find that axial viscous diffusion greatly precipitates the upper occlusion bound $\operatorname{Re}_{\max }$ (by $74 \%$ for the high-viscosity silicone oil). When the viscosity is low (low-viscosity silicone oil), inertia cannot be neglected and is found to precipitate $\operatorname{Re}_{\max }$ by $20 \%$. We also find that these two effects determine whether the occlusion mechanism is dictated by absolute linear instability or the loss of travelling-wave solutions.

Our model also accounts for full inter-phase coupling between the liquid and gas, and this has allowed us to study the effect of a laminar counter-current gas flow on the occlusion bound $\mathrm{Re}_{\max }$. For low-viscosity liquids, where the wave-induced pressure variation in the gas becomes relevant in the liquid force balance, we find that $\operatorname{Re}_{\max }$ is significantly reduced with increasing $\left|R_{\mathrm{g}}\right|$ (by up to $25 \%$ in our computations). Thereby, the conditions we have studied differ from previous works. Alekseenko et al. (2009) experimentally studied the linear stability of a falling liquid film subject to a co-current turbulent gas flow. Their tube radius was large and thus occlusion could not occur. Camassa et al. $(2012,2017)$ studied co-current upward liquid/gas flows based on a longwave model, where the effect of the (turbulent) gas flow was accounted for by relaxing the inter-phase coupling conditions in the limit of high gas velocities. By contrast, we focus on laminar (counter-current) gas flows, where our model, which relies on the unrelaxed coupling conditions, behaves well.

For the case of a planar falling liquid film sheared by a confined gas flow, many works have investigated the flooding phenomenon, where the liquid film either occludes the channel (Vlachos et al. 2001), surface waves (Tseluiko \& Kalliadasis 2011) or the liquid flow (Trifonov 2010) reverse direction, or the liquid film disintegrates into drops (Zapke \& Kröger 2000).

Finally, several works are related less directly to the studied problem. Kouris \& Tsamopoulos (2001) studied liquid/liquid gravity-driven flows through a vertical pipe. Beltrame (2018) studied the transition between partial and complete wetting in a microtube. Xu \& Jensen (2017) studied migration of liquid films in tubes with rough walls. Moreover, many works have dealt with pressure-driven core-annular flows (Aul \& Olbricht 1990; Joseph et al. 1997) or liquid films falling down fibres (Kalliadasis \& Chang 1994; Quéré 1999). In the latter case, occlusion cannot occur, unless the gas phase is confined by an additional concentric hollow cylinder (Wray 2013). 


.

Our manuscript is structured as follows. Section 2 introduces the governing equations, and details of our model (§2.1), direct numerical simulations ( $\$ 2.2)$, and linear stability calculations $(\S 2.3)$. Results are presented in $\S 3$, where we start by proving that occlusion in real systems results from surface waves that lie beyond the limit of travelling-wave solutions (§3.1). In $\S 3.2$, we compute the upper and lower occlusion bounds $\operatorname{Re}_{\max }$ and $\mathrm{Re}_{0}$, delimiting the regimes of certain, conditional, and impossible occlusion. For one of the studied cases, we validate these results based on our own DNS of travelling-wave solutions. In sections 3.3 to 3.6 , we show how $\mathrm{Re}_{\max }$ is affected by gravity, axial viscous diffusion, inertia, and the core gas flow. In $\S 3.7$, we demonstrate occlusion scenarios I (certain occlusion regime) and II (conditional occlusion regime) by reproducing two of the experimental runs from Dao \& Balakotaiah (2000) with spatio-temporal computations. In $§ 3.8$, we show that occlusion in the conditional regime can be prevented through coherent inlet forcing. Conclusions are drawn in $\S 4$. The appendix reports comparisons with transient DNS (appendix A), numerical details regarding our travelling-wave DNS (appendix B), as well as additional discussions of panels 13a (appendix C) and 4a (appendix D). Finally, appendix E introduces an augmented version of our WRIBL model that allows a better representation of liquid plugs, based on a localized force term.

\section{Mathematical description}

We consider the flow in panel 1a, which is rotationally symmetric w.r.t. the tube axis, and is governed by the Navier-Stokes and continuity equations for the liquid (subscript $k=\mathrm{l}, \chi_{\mathrm{l}}=1$ ) and gas (subscript $\left.k=\mathrm{g}, \chi_{\mathrm{g}}=\Pi_{\mu} / \Pi_{\rho}\right)$ :

$$
\begin{gathered}
\mathrm{d}_{t} u_{k}=-\partial_{x} p_{k}+\operatorname{Fr}^{-2}+\chi_{k} \operatorname{Re}^{-1}\left\{r^{-1} \partial_{r}\left(r \partial_{r} u\right)+\partial_{x x} u\right\}, \\
\mathrm{d}_{t} v_{k}=-\partial_{r} p_{k}+\chi_{k} \operatorname{Re}^{-1}\left\{\partial_{r}\left[r^{-1} \partial_{r}(r v)\right]+\partial_{x x} v\right\},
\end{gathered}
$$

$$
\partial_{x} u_{k}+r^{-1} \partial_{r}\left(v_{k} r\right)=0
$$

where $\operatorname{Re}=\rho_{\mathrm{l}} \mathcal{U} \mathcal{L} / \mu_{\mathrm{l}}=q_{10}^{\star} /\left(\pi R^{\star}\right) /\left(\mu_{\mathrm{l}} / \rho_{\mathrm{l}}\right)$ is the Reynolds number and $\operatorname{Fr}=\mathcal{U} / \sqrt{g \mathcal{L}}$ is the Froude number, all variables having been rendered dimensionless with the following reference scales: the length scale $\mathcal{L}=R^{\star}$, which corresponds to the tube radius, the velocity scale $\mathcal{U}=q_{10}^{\star} / \pi / R^{\star 2}$, which corresponds to the surface velocity of the liquid, the time scale $\mathcal{T}=\mathcal{L} / \mathcal{U}$, and the pressure scale $\mathcal{P}_{k}=\rho_{k} \mathcal{U}^{2}$, which corresponds to the phase-specific dynamical pressure. The star symbol denotes dimensional quantities, and the subscript zero the flat-film primary flow. Thus, $q_{10}$ designates the nominal liquid flow rate.

The set of governing equations is completed by the boundary conditions:

$$
\left.u_{\mathrm{l}}\right|_{r=R}=\left.v_{\mathrm{l}}\right|_{r=R}=0,\left.\partial_{r} u_{\mathrm{g}}\right|_{r=0}=\left.v_{\mathrm{g}}\right|_{r=0}=0,
$$

and the kinematic/dynamic inter-phase coupling conditions at the film surface $r=d$ :

$$
u_{\mathrm{l}}=u_{\mathrm{g}}, v_{\mathrm{l}}=v_{\mathrm{g}}=\partial_{t} d+u_{\mathrm{k}} \partial_{x} d,
$$

$$
\begin{gathered}
\tau_{1}^{\mathrm{t}}=\Pi_{\mu} \tau_{\mathrm{g}}^{\mathrm{t}}, \\
\tau_{k}^{\mathrm{t}}=\partial_{r} u_{k}+\partial_{x} v_{k}-2 \partial_{x} d\left(1+\partial_{x} d^{2}\right)^{-1}\left[\partial_{x} u_{k}-\partial_{r} v_{k}\right],
\end{gathered}
$$

$$
\begin{gathered}
\tau_{1}^{\mathrm{n}}+\operatorname{Re} p_{\mathrm{l}}=\Pi_{\mu} \tau_{\mathrm{g}}^{\mathrm{n}}+\Pi_{\rho} \operatorname{Re} p_{g}+\operatorname{ReWe}^{-1} \kappa \\
\tau_{k}^{\mathrm{n}}=2\left(1+\partial_{x} d^{2}\right)^{-1}\left[\partial_{x} d \partial_{r} u_{k}-\partial_{r} v_{k}-\partial_{x} d^{2} \partial_{x} u_{k}+\partial_{x} d \partial_{x} v_{k}\right],
\end{gathered}
$$

where $\tau^{\mathrm{t}}$ and $\tau^{\mathrm{n}}$ designate the tangential and normal interfacial viscous stresses, $\Pi_{\rho}=\rho_{\mathrm{g}} / \rho_{\mathrm{l}}$ and $\Pi_{\mu}=\mu_{\mathrm{g}} / \mu_{\mathrm{l}}$ are the density and dynamic viscosity ratios, We $=\rho_{\mathrm{l}} \mathcal{U}^{2} \mathcal{L} / \sigma$ is 
the Weber number, and $\kappa$ denotes the total curvature of the film surface:

$$
\kappa=\partial_{x x} d-\frac{1}{d}\left[1-\frac{1}{2}\left(\partial_{x} d\right)^{2}\right] .
$$

We will refer to computations based on the full equations (2.1) to (2.3) as direct numerical simulations (DNS). These have been performed for validation purposes and results are reported in appendix A and panel 5d. However, most of our computations were carried out with the weighted residual integral boundary layer (WRIBL) model of Dietze \& Ruyer-Quil (2015), which we introduce next.

\subsection{Weighted residual integral boundary layer (WRIBL) model}

The WRIBL model consists of three coupled partial differential equations for the core radius $d$, and the flow rates $q_{1}$ and $q_{\mathrm{g}}$ (Dietze \& Ruyer-Quil 2015):

$$
\partial_{x} q_{j}-\varepsilon_{j} 2 \pi d \partial_{t} d=0
$$

$$
\begin{aligned}
\left\{S_{i} \partial_{t} q_{i}+F_{i j}\right. & \left.q_{i} \partial_{x} q_{j}+G_{i j} q_{i} q_{j} \partial_{x} d\right\}=\operatorname{Fr}^{-2}\left(1-\Pi_{\rho}\right) \\
& -\mathrm{We}^{-1} \partial_{x}[\kappa]+\operatorname{Re}^{-1}\left(C_{j l}-\Pi_{\mu} C_{j g}\right) q_{j} \\
& +\operatorname{Re}^{-1}\left\{J_{j} q_{j}\left(\partial_{x} d\right)^{2}+K_{j} \partial_{x} q_{j} \partial_{x} d+L_{j} q_{j} \partial_{x x} d+M_{j} \partial_{x x} q_{j}\right\},
\end{aligned}
$$

where the subscripts $i$ and $j$ are to be permuted over the phase indicators for the liquid $(i, j=\mathrm{l})$ and gas $(i, j=\mathrm{g})$. This yields two equations from $(2.5 a)$, ensuring integral mass conservation, and one equation from $(2.5 b)$, which ensures conservation of momentum. The RHS terms in $(2.5 b)$ account for different driving forces, i.e. gravity, capillarity, and viscous drag. Relating these terms directly to one another yields two alternative dimensionless groups that are also useful for characterizing the studied flow regimes: the Bond number $\mathrm{Bo}=\mathrm{We} \mathrm{Fr}^{-2}=\rho_{1} g R^{\star 2} / \sigma$, which relates gravity to capillarity, and the capillary number $\mathrm{Ca}=\mathrm{We} / \mathrm{Re}=\mu_{\mathrm{l}} \mathcal{U} / \sigma$, which relates viscous drag to capillarity. Following Dao \& Balakotaiah (2000), we will also make use of the Kapitza number $\mathrm{Ka}=\sigma \rho_{1}^{-1} g^{-1 / 3} \mu_{1}^{-4 / 3}$, and the Laplace number $\mathrm{La}=\sigma \rho_{1} R^{\star} / \mu_{1}^{2}$. We point out however that the list Re, Fr, We, $\mathrm{Bo}, \mathrm{Ca}, \mathrm{Ka}$, and La, contains only three independent dimensionless groups, e.g. Re, Ka, and Bo, which (along with $\Pi_{\mu}$ and $\Pi_{\rho}$ ) suffice to fully describe the flow. All other groups can be expressed in terms of these three, e.g. $\mathrm{Ca}=\operatorname{Re}\left(\mathrm{BoKa}^{3}\right)^{-1 / 2}$.

The model is completed by an equation for the gas pressure gradient $\left.\partial_{x} p_{\mathrm{g}}\right|_{r=d}$ :

$$
\begin{aligned}
\left.2 \Pi_{\rho} \operatorname{Re} \partial_{x} p_{\mathrm{g}}\right|_{r=d}= & -\operatorname{Re}\left\{\underline{\mathrm{S}}_{i} \partial_{t} q_{i}+\underline{\mathrm{F}}_{i j} q_{i} \partial_{x} q_{j}+\underline{\mathrm{G}}_{i j} q_{i} q_{j} \partial_{x} d\right\} \\
& +\operatorname{Re} \operatorname{Fr}^{-2}\left(1+\Pi_{\rho}\right)-\mathrm{We}^{-1} \operatorname{Re} \partial_{x}[\kappa]+\left(C_{j l}+\Pi_{\mu} C_{j g}\right) q_{j} \\
& +\underline{\mathrm{J}}_{j} q_{j}\left(\partial_{x} d\right)^{2}+\underline{\mathrm{K}}_{j} \partial_{x} q_{j} \partial_{x} d+\underline{\mathrm{L}}_{j} q_{j} \partial_{x x} d+\underline{\mathrm{M}}_{j} \partial_{x x} q_{j},
\end{aligned}
$$

which is used either to evaluate the pressure distribution a posteriori or to impose a gas pressure drop through an integral condition. The coefficients $F_{i j}, G_{i j}, C_{i j}, S_{j}, J_{j}, K_{j}$, and $M_{j}$ in $(2.5 b)$ and their counterparts marked by an underscore in $(2.5 c)$ are known functions of $d$. They can be found in appendix A of Dietze \& Ruyer-Quil (2015).

We solve the equation system (2.5) numerically using three approaches: (i) transient periodic computations, where the domain length $L$ corresponds to the wavelength $\Lambda$ and where $\left.\partial_{x}^{i} q_{k}\right|_{x=0}=\left.\partial_{x}^{i} q_{k}\right|_{x=\Lambda}$ and $\left.\partial_{x}^{i} d\right|_{x=0}=\left.\partial_{x}^{i} d\right|_{x=\Lambda}$ for $i=0,1,2,3$. The code employed for this is based on a Crank-Nicolson time discretization and first-order central differences for spatial discretization (Dietze \& Ruyer-Quil 2015); (ii) continuation of periodic travelling wave solutions that are stationary in the wave-fixed coordinate $\xi=x$-ct moving with the wave celerity $c$. These computations were performed with the continuation software AUTO07P (Doedel 2008); (iii) transient open-domain computations with inlet and outlet 
conditions. At the liquid inlet, $x=0$, we fix the core radius $d=d_{0}$ of the flat-film primary flow. For the liquid flow rate $q_{1}$, we superimpose a zero-mean temporal perturbation on the nominal value $q_{10}$ :

$$
q_{1}(x=0, t)=q_{10}[1+F(t)],
$$

where the function $F(t)$ defines the type of inlet perturbation:

$$
F(t)=\epsilon_{1} \sin (2 \pi f t)+\epsilon_{2} \sum_{k=1}^{N} \sin \left(2 \pi k \Delta f t+\varphi_{\text {rand }}\right), \quad \Delta f=2 f_{\mathrm{c}} / N .
$$

The first term in (2.6b) constitutes a harmonic perturbation of frequency $f$ and the second one mimics white noise through a series of $N=1000$ Fourier modes that are shifted by a random phase shift $\varphi_{\text {rand }}=\varphi_{\text {rand }}(k) \in[0,2 \pi]$ and that span a frequency range of twice the linear cut-off frequency $f_{\mathrm{c}}$ (Chang et al. 1996a). All our computations were run with the same $\varphi_{\text {rand }}(k)$ number series, which was generated once and for all with the pseudo random number generator RandomReal in Mathematica (2014). The strength of the two terms in (2.6b) is determined through their amplitudes $\epsilon_{1}$ and $\epsilon_{2}$. When $\epsilon_{1}=0$, the inlet perturbation consists only of white noise. This setting will be used to simulate the natural, noise-driven, evolution of a wavy film as it would occur in an experiment (sections 3.1 and 3.7). In $\S 3.8$, we will use coherent inlet forcing to prevent occlusion. In those computations, we will set $\epsilon_{1}>0$, thus adding a monochromatic harmonic perturbation to the inlet noise.

At the outlet, $x=L$, we have implemented the boundary conditions of Richard et al. (2016), which ensure that liquid is always sufficiently drained from the domain. We set $\left.d\right|_{N+2}=\left.d\right|_{N+1}=\left.d\right|_{N}$, and $\left.q_{1}\right|_{N+1}=q_{10}\left(R-\left.d\right|_{N}\right)^{3 / 2} /\left(R-d_{0}\right)^{3 / 2}$, where $N$ corresponds to the last grid point within the domain and $N+1, N+2$ to the two downstream ghost points. Computations were started from the initial condition $d(x, t=0)=d_{0}, q_{1}(x, t=0)=q_{10}$.

In our codes, the gas flow rate $q_{\mathrm{g}}$ is expressed in terms of the total flow rate $q_{\text {tot }}$ :

$$
q_{\text {tot }}(t)=q_{1}(x, t)+q_{\mathrm{g}}(x, t),
$$

which is spatially invariant. In our computations, we either fix $q_{\text {tot }}$ explicitly, which fixes the nominal gas flow rate $q_{\mathrm{g} 0}$, given a nominal liquid flow rate $q_{10}$. Or, $q_{\text {tot }}$ is dynamically adjusted to produce a fixed gas pressure drop $\Delta p_{\mathrm{g}}$, imposed through an integral condition on $(2.5 c)$. We quantify this pressure drop through the normalized pressure gradient:

$$
\Psi=\Delta p_{\mathrm{g}} / L \mathrm{Fr}^{2},
$$

where $L$ is the domain length. When $\Psi=1$, the pressure drop exactly balances the weight of the gas column, and we refer to this as the aerostatic situation. This is most realistic for reproducing experiments where the core gas flow is quiescent.

In our transient computations, the wavy liquid film can tend toward occluding the tube. In an experiment, such an event would form a liquid plug separated by two gas bubbles. The topological change occurring in this situation cannot be mathematically represented by our model, which implies continuous fluid layers. Thus, we apply a numerical procedure to allow our computations to continue beyond such events. For this, we level the core radius $d$ to an arbitrary value $d_{\text {crit }}=0.1$ whenever / wherever $d \leqslant d_{\text {crit }}$. As a result, occluded zones are represented by a thin gas filament connecting two bubbles either side of a liquid plug (figure 3c). Although our approach allows to recover some basic features of these zones, it is a crude and invasive approximation of the actual physics. For example, every time $d$ is reset to $d_{\text {crit }}$, a bit of liquid mass is lost and this can cause the unphysical reopening of liquid plugs (supplementary movies M2 and M3). We stress however, that we are not interested in studying the actual behaviour of occluded zones here. Our current 
paper focusses on the events leading up to occlusion, and thus our numerical limitation of the core radius $d$ is simply a means to continue our computations until a developed state has been reached. Nonetheless, we propose in appendix E an alternative way of representing liquid plugs in the framework of our WRIBL model, based on an additional repulsive force in the momentum equation $(2.5 b)$ that stabilizes the film surface at very small $d$. This approach is inspired by the representation of contact line problems with film models (Thiele et al. 2001), and confronting it with full-fledged plug models (Ubal et al. 2008; Suresh \& Grotberg 2005) is an enticing prospect for future work.

\subsection{Direct numerical simulation (DNS)}

We have validated our model computations with selected DNS based on the full governing equations (2.1)-(2.4). These were performed with two different codes.

Transient simulations were performed with the finite volume solver Gerris (Popinet 2009), which uses the volume of fluid (Hirt \& Nichols 1981) and continuum surface force (Brackbill et al. 1992) methods to represent the two-phase nature of the flow. We have successfully applied this code to falling liquid films in previous works (Dietze \& Ruyer-Quil 2013, 2015; Dietze 2019), where further details are given. In the current work, we have used it to perform axisymmetric transient DNS on a domain spanning the wavelength $\Lambda$ in streamwise direction and the tube radius $R$ in radial direction, applying periodic streamwise boundary conditions. To allow imposing the gas pressure drop $\Delta p_{\mathrm{g}}$ (2.8) in this periodic setting, the streamwise momentum equations (2.1) for the liquid $(k=\mathrm{l})$ and gas $(k=\mathrm{g})$ were rewritten in terms of the modified pressure $\tilde{p}_{k}=p_{k}+x \Delta p_{k} / \Lambda$, yielding the additional source term $\Gamma_{k}$ :

$$
\Gamma_{k}=\frac{\Delta p_{k}}{\Lambda}, \quad \Delta p_{\mathrm{l}}=\Pi_{\rho} \Delta p_{\mathrm{g}} .
$$

Thanks to this source term, the governing equations can be solved with a periodicity condition on $\tilde{p}_{k}$, while the actual pressure $p_{k}$ is subject to a pressure drop, allowing to control the gas flow rate. Our transient DNS were started from a flat film initial condition corresponding to the primary flow. All transient DNS were performed for case 4 in table 1 , on a grid of square cells with increments $\Delta_{x}=\Delta_{r}=2^{-7}$ in the bulk of the fluid phases and $\Delta_{x}=\Delta_{r}=2^{-8}$ around the liquid-gas interface. The time step $\Delta_{t}$ was dynamically adapted so that $w^{\star} \Delta_{t} / \Delta_{x} \leqslant 0.8$ in each cell, whereby $w^{\star}$ denotes the magnitude of the local dimensional velocity. We have verified that our simulations are grid independent. Results of our transient DNS are presented in appendix A, where they are used to validate our model computations.

The second DNS code allows to construct travelling-wave solutions of (2.1)-(2.4) based on a pseudo-spectral approach, which we have integrated into the continuation software AUTO07P (Doedel 2008). This has allowed us to verify our numerical continuation results obtained with the WRIBL model (see panel 5d). In these travelling-wave DNS, the gas phase is not accounted for, i.e. $\Pi_{\mu}=\Pi_{\rho}=0$, and thus we have only applied them to case 3 , where the effect of the gas is negligible. The underlying numerical procedure is detailed in appendix $\mathrm{B}$.

\subsection{Linear stability analysis}

Linearizing the WRIBL model (2.5) around the uniform base state $\left\{d, q_{k}\right\}=\left\{d_{0}, q_{k 0}\right\}$ and assuming a small-amplitude perturbation $\left\{d^{\prime}, q_{k}^{\prime}\right\}$ :

$$
\begin{aligned}
d(x, t) & =d_{0}+d^{\prime}(x, t)=d_{0}+\hat{d} \exp [i(\alpha x-\omega t)] \\
q_{k}(x, t) & =q_{k 0}+q_{k}^{\prime}(x, t)=q_{k 0}+\hat{q}_{k} \exp [i(\alpha x-\omega t)],
\end{aligned}
$$


(a)

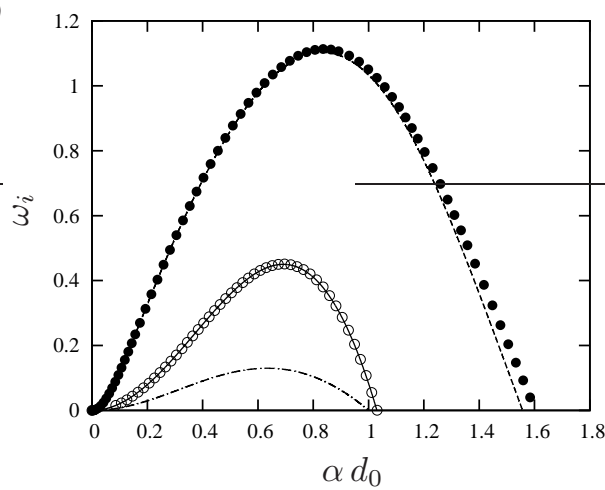

(c)

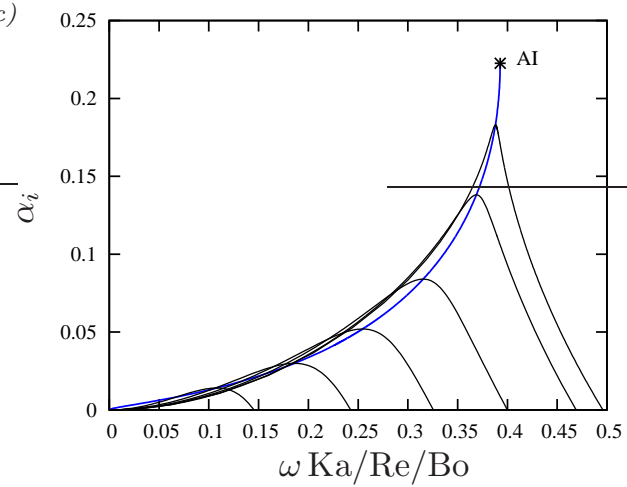

(b)

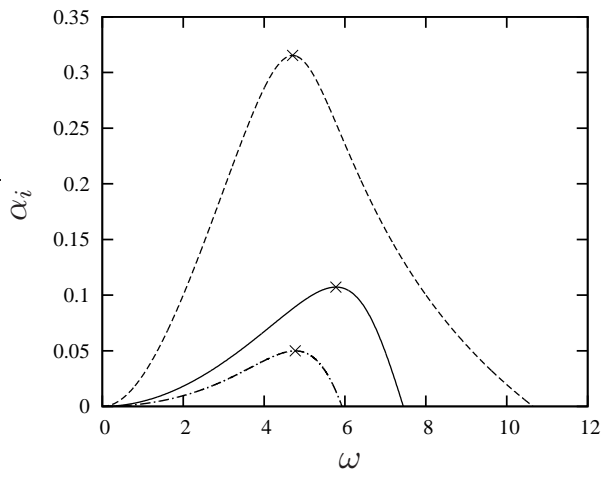

(d)

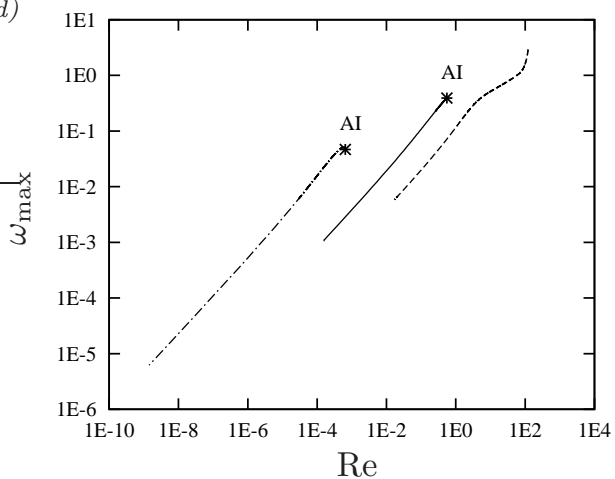

Figure 2. Linear instability predictions for three cases from table 1: case 1 (dot-dashed lines, diamonds); case 3 (solid lines, open circles); case 4 (dashed lines, filled circles). The core fluid is subject to an aerostatic pressure gradient $\Psi=1$. Lines correspond to the model dispersion relation (2.11). (a) Temporal growth: $\alpha \in \mathbb{R}, \omega=\omega_{r}+i \omega_{i}$ in (2.10). Symbols correspond to Orr-Sommerfeld solution, following the work of Hickox (1971); (b) spatial growth: $\omega \in \mathbb{R}, \alpha=\alpha_{r}+i \alpha_{i}$ in (2.10). Crosses highlight maximal spatial growth; (c) dispersion curves under increasing Re $\in[0.1,0.54]$. Blue line traces maximum growth rate $\alpha_{i}\left(\omega_{\max }\right) ;(d)$ most amplified angular velocity $\omega_{\max }$ versus Re. Asterisks mark absolute linear instability threshold (AI).

yields the complex dispersion relation:

$$
\begin{aligned}
\operatorname{DR}(\omega, \alpha)= & 2 \pi d_{0} \omega\left\{\varepsilon_{j l} C_{j l}+\varepsilon_{j g} \Pi_{\mu} C_{j g}\right\}+\alpha\left\{\partial_{d} C_{j l} q_{j 0}-\Pi_{\mu} \partial_{d} C_{j g} q_{j 0}\right\} \\
& -2 \pi d_{0} \alpha^{2} M_{j} \varepsilon_{j l}-\alpha^{3} L_{j} q_{j 0}-i \operatorname{ReWe}\left\{\alpha^{2} / d_{0}^{2}-\alpha^{4}\right\} \\
& +i \operatorname{Re}\left\{2 \pi d_{0}\left[\omega^{2}\left(S_{\mathrm{g}}-S l\right)+\omega \alpha\left(F_{j l} q_{j 0}-F_{j g} q_{j 0}\right)\right]-\alpha^{2} G_{j k}\right\},
\end{aligned}
$$

where $j$ and $k$ are to be permuted over the phase indicators 1 and $\mathrm{g}, \omega$ designates the (complex) wave frequency, $\alpha$ the (complex) wave number, $\varepsilon_{l l}=\varepsilon_{\mathrm{gg}}=-1$, and $\varepsilon_{\mathrm{lg}}=\varepsilon_{\mathrm{gl}}=1$.

A temporal stability analysis assumes that waves grow self-similarly in their reference frame. This description implies $\alpha \in \mathbb{R}$ and $\omega=\omega_{r}+i \omega_{i}$, where the temporal growth rate $\omega_{i}$ is obtained by solving $\operatorname{DR}(\omega, \alpha)=0$. In panel $2 \mathrm{a}$, we have plotted the thus obtained dispersion curve $\omega_{i}(\alpha)$ of the temporal growth rate for cases 1,3 and 4 from table 1 . Lines correspond to the model dispersion relation (2.11), and symbols correspond to our own numerical solutions of the Orr-Sommerfeld equations, following the work of Hickox (1971). Agreement between the two data sets is good. Thus, our model is able to accurately represent the linear wave selection.

In a spatially-evolving film, it is more representative to consider the spatial growth rate 
$\alpha_{i}$. We thus solve $\operatorname{DR}(\omega, \alpha)=0$ for $\alpha_{i}(\omega)$, by assuming $\omega \in \mathbb{R}$ and $\alpha=\alpha_{r}+i \alpha_{i}$. In panel $2 \mathrm{~b}$, we have plotted the thus obtained growth rate dispersion curves $\alpha_{i}(\omega)$ for the three cases from panel 2a. Crosses mark the point of maximal growth rate and we denote $\omega_{\max }$ the associated most-amplified angular velocity.

In section 3.2, we will characterize occlusion by determining the limit of travellingwave solutions through numerical continuation. This limit is very sensitive to the wave frequency $f$. Thus we focus on the spatially most-amplified waves, which are most likely to emerge from linear wave selection in an experiment. For this, we impose the linearly most-amplified frequency $f_{\max }=2 \pi / \omega_{\max }$ in our continuation runs.

To determine $f_{\max }$, which changes with the control parameters varied in these runs, our continuation code was augmented to additionally solve for $\omega_{\max }$ subject to:

$$
\operatorname{DR}\left(\omega_{\max }, \alpha\right)=0,\left.\quad \partial_{\omega} \alpha_{i}\right|_{\omega=\omega_{\max }}=0,
$$

where $\partial_{\omega} \alpha_{i}$ is obtained from $\partial_{\omega} \mathrm{DR}=0$. In a given continuation run, the linear stability problem (2.12) is solved for the primary flow $\left\{q_{10}, q_{\mathrm{g} 0}\right\}=\left\{\bar{q}_{\mathrm{l}}, \bar{q}_{\mathrm{g}}\right\}$, which is fixed by the mean flow rates $\bar{q}_{1}$ and $\bar{q}_{\mathrm{g}}$ of the current travelling wave solution.

As shown by Camassa et al. (2014), the annular falling liquid film can be subject to absolute instability. Thus, we have checked for the absolute instability threshold (AI) in all our continuation runs. In the context of our spatial stability analysis, where $\omega \in \mathbb{R}$, the AI threshold bounds the solutions of (2.12) in terms of the varied control parameter, e.g. Re, because the maximum growth rate $\alpha_{i}\left(\omega_{\max }\right)$ diverges there. For example, panel $2 \mathrm{c}$ represents dispersion curves $\alpha_{i}(\omega)$ for case 3 at different values of Re, increased toward the AI threshold. The latter is marked by an asterisk on the blue curve, which traces the locus of the spatial growth rate maximum $\alpha_{i}\left(\omega_{\max }\right)$. We point out that $\omega$ has been rescaled in panel 2c.

Panel $2 \mathrm{~d}$ represents how $\omega_{\max }$ changes with Re for the three cases from panel $2 \mathrm{~b}$. These curves allow to discern the AI threshold (marked by asterisks) in terms of Re. For case 4 (dashed curve), we have truncated our solution at $R e=100$, beyond which our nonlinear model predictions are no longer reliable. The AI threshold lies beyond this point but, as we will see, the occlusion bound $\mathrm{Re}_{\max }$ lies well within the represented range (panel 6a).

In our current study, we focus on regimes where Bo $\geqslant 1$. In this case, the AI threshold is always situated beyond the limit of nonlinear travelling-wave solutions and thus it does not affect the occlusion bound. However, we will demonstrate that neglecting certain physical effects in the mathematical description, such as axial viscous diffusion (panel $9 \mathrm{~b}$ ) or inertia (panel 12a), can change this.

\section{Results and discussion}

In subsections 3.1 and 3.2, we reproduce numerically the visualization experiments of Camassa et al. (2014) and demonstrate that the occlusion of a narrow tube by a wavy falling liquid film is caused by surface waves that do not possess a finite-amplitude travelling state. Depending on how these waves emerge in a spatially evolving film, either directly from linear instability (scenario I), or subsequent nonlinear dynamics (scenario II), different occlusion scenarios are possible. In subsection 3.2, we determine the bounds of these two scenarios based on the numerical continuation of travellingwave solutions with our model (2.5). We obtain an upper conservative bound $\mathrm{Re}_{\max }$, above which occlusion in a naturally evolving film is certain, and a lower conservative bound $\mathrm{Re}_{0}$, below which occlusion is impossible. These bounds delimit a regime of conditional occlusion, $\operatorname{Re}_{0}<\operatorname{Re}<\operatorname{Re}_{\max }$, where occlusion is theoretically possible but does not necessarily occur in a real system. 


\begin{tabular}{cccccccc} 
Case & $\rho_{\mathrm{l}}\left(\mathrm{kg} / \mathrm{m}^{3}\right)$ & $\mu_{\mathrm{l}}(\mathrm{Pas})$ & $\sigma(\mathrm{mN} / \mathrm{m})$ & $R^{\star}(\mathrm{mm})$ & $\mathrm{Ka}(-)$ & $\Pi_{\rho}(-)$ & $\Pi_{\mu}(-)$ \\
\hline 1 & 970 & 12.9 & 21.5 & 5.0 & $3.3 \times 10^{-3}$ & $1.2 \times 10^{-3}$ & $1.4 \times 10^{-6}$ \\
2 & 1260.7 & 1.049 & 64.0 & 4.765 & 0.30 & $0.95 \times 10^{-3}$ & $1.7 \times 10^{-5}$ \\
3 & 1223.9 & 0.167 & 65.0 & 3.175 & 3.53 & $0.98 \times 10^{-3}$ & $1.1 \times 10^{-4}$ \\
4 & 900 & 0.0045 & 20.0 & 1.5 & 121.4 & $1.3 \times 10^{-3}$ & $4.0 \times 10^{-3}$ \\
\hline \multirow{2}{*}{ Case } & $\mathrm{Re}_{\max }(-)$ & $\mathrm{Fr}(-)$ & $\mathrm{We}(-)$ & Bo (-) & $\mathrm{La}(-)$ & $\mathrm{Ca}(-)$ & figures \\
\hline \multirow{2}{*}{1} & $1.91 \times 10^{-4}$ & $2.7 \times 10^{-3}$ & $8.4 \times 10^{-5}$ & 11.06 & $6.3 \times 10^{-4}$ & 0.37 & $3-5,9,11$ \\
2 & 0.038 & 0.040 & $7.1 \times 10^{-3}$ & 4.39 & 0.35 & 0.142 & 5,15 \\
3 & 0.39 & 0.063 & $7.3 \times 10^{-3}$ & 1.86 & 9.1 & 0.028 & $5,9,15,16,18$ \\
4 & 18.9 & 0.42 & 0.18 & 0.993 & $1.3 \times 10^{3}$ & 0.012 & $6,9,12-14,17-19$
\end{tabular}

TABLE 1. Representative parameters for our computations. Liquid properties correspond to a high-viscosity silicone oil (case 1), glycerol-water mixtures concentrated at $99 \%$ and $89 \%$ by mass (cases 2 and 3), and a low-viscosity silicone oil (case 4), while the core fluid is air with $\mu_{\mathrm{g}}=1.8 \cdot 10^{-5}$ Pas and $\rho_{\mathrm{g}}=1.2 \mathrm{~kg} / \mathrm{m}^{3}$. Case 1 corresponds to figure $3 \mathrm{a}$ in Camassa et al. (2014), and cases 2 and 3 correspond to experimental runs 20 and 13 in Dao \& Balakotaiah (2000). The dimensionless groups are $\mathrm{Bo}=\rho_{1} g \mathcal{L}^{2} / \sigma, \mathrm{Ca}=\mu_{1} \mathcal{U} / \sigma, \mathrm{Re}=\rho_{1} \mathcal{U L} / \mu_{1}, \mathrm{Ka}=\sigma \rho_{1}^{-1} g^{-1 / 3} \mu_{1}^{-4 / 3}$, $\mathrm{La}=\sigma \rho_{1} \mathcal{L} / \mu_{1}^{2}, \mathrm{We}=\rho_{1} \mathcal{U}^{2} \mathcal{L} / \sigma$, and $\mathrm{Fr}=\mathcal{U} / \sqrt{g \mathcal{L}}$, where $\mathcal{L}=R^{\star}$ and $\mathcal{U}=q_{10}^{\star} / \pi / \mathcal{L}^{2}, q_{10}^{\star}$ designating the dimensional nominal liquid flow rate. $\operatorname{Re}_{\max }$ is the upper conservative occlusion bound (see e.g. panels $5 \mathrm{a}$ and $5 \mathrm{~b})$.

In subsection 3.7, we demonstrate occlusion scenarios I and II, which are associated with the regimes of certain $\left(R e>R e_{\max }\right)$ and conditional occlusion $\left(R_{0}<R e<R e_{\max }\right)$, by reproducing two of the experiments in Dao \& Balakotaiah (2000) with our own spatiotemporal computations. Before that, in subsections 3.3 to 3.6, we establish how different physical effects, i.e. gravity, axial viscous diffusion, inertia, and the core gas flow, affect the upper conservative bound $\mathrm{Re}_{\max }$, which delimits these two regimes. Except for section 3.6, where we will vary the gas Reynolds number $\mathrm{Re}_{\mathrm{g}}$, our computations have been performed for an aerostatic pressure drop, i.e. $\Psi=1$. This is most representative of experiments in a quiescent gas.

Parameters for our computations are varied around the four cases listed in table 1, which represent real liquids and plausible values of the tube radius. Case 1 corresponds to figure 3a in Camassa et al. (2014), where the working liquid was a high-viscosity silicone oil. Cases 2 and 3 correspond to experimental runs 20 and 13 in Dao \& Balakotaiah (2000), where glycerol-water mixtures concentrated at $99 \%$ and $89 \%$ by mass were used. Finally, case 4 concerns a liquid film of low-viscosity silicone oil. The four cases cover a considerable range of the Laplace number $\mathrm{La}=\sigma \rho_{\mathrm{l}} R^{\star} / \mu_{1}^{2}$, which relates capillarity to viscous drag, and the Reynolds number, which we have quantified with $\operatorname{Re}_{\max }$. We see that inertia, which drives the Kapitza instability, is significant for case 4. For this case, which is the most challenging, we have validated our model computations with transient DNS in appendix A (comparisons with travelling-wave DNS, for case 3, are reported in panel $5 \mathrm{~d})$.

\subsection{Nature of the surface waves that cause occlusion}

Camassa et al. (2014) performed occlusion experiments in an $R^{\star}=0.5 \mathrm{~mm}$ and $L^{\star}=400$ $\mathrm{mm}$ transparent tube using high-viscosity silicone oil (case 1 in table 1 ). In their figure 3 , the authors produce photographs of the wavy falling liquid film at different Re beyond the 
(a)

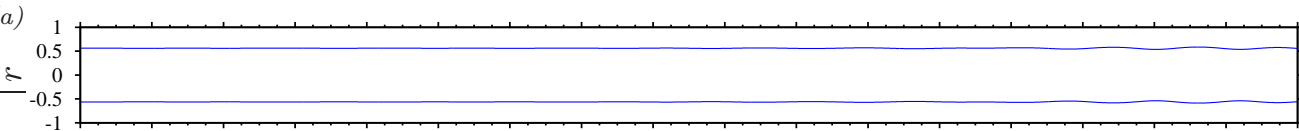

(b) 1
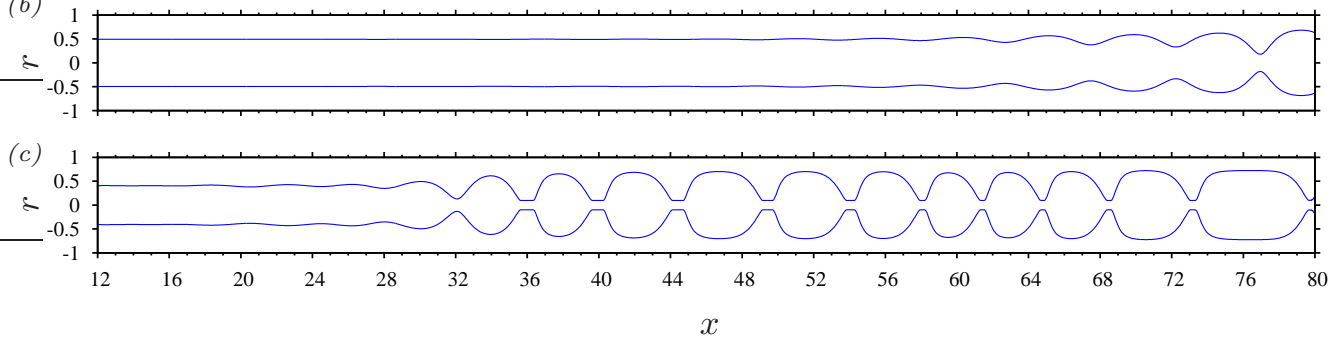

Figure 3. Spatio-temporal computations of the experiments in panels 3a, 3b, and 3c of Camassa et al. (2014), which were performed in an $L^{\star}=400 \mathrm{~mm}$ tube. Parameters correspond to case 1 in table 1. Computations were performed with our model (2.5) using inlet/outlet conditions and the noisy inlet perturbation (2.6), where we have set $\epsilon_{1}=0$ and adjusted $\epsilon_{2}$ to match the experimental wave growth. (a) $\mathrm{Re}=2.3 \times 10^{-4}, q_{10}^{\star}=4.8 \times 10^{-2} \mathrm{~cm}^{3} / \mathrm{s}, \epsilon_{2}=0.0002$; (b) $\mathrm{Re}=3.2 \times 10^{-4}$, $q_{10}^{\star}=6.7 \times 10^{-2} \mathrm{~cm}^{3} / \mathrm{s}, \epsilon_{2}=0.00005$; (c) $\mathrm{Re}=4.5 \times 10^{-4}, q_{10}^{\star}=9.4 \times 10^{-2} \mathrm{~cm}^{3} / \mathrm{s}, \epsilon_{2}=0.0001$.

experimental occlusion threshold, allowing to discern the nature of the surface waves that cause occlusion. We have reproduced three of these experiments with spatio-temporal computations based on our model (2.5) on a long domain using inlet/outlet conditions (see section 2.1). Experimental noise was mimicked through the noisy inlet perturbation (2.6), where we have set $\epsilon_{1}=0$, and tuned $\epsilon_{2}$ to match the spatial evolution of surface waves in the experiments (when decreasing/increasing $\epsilon_{2}$, the region of linear growth of surface waves is stretched/compressed in streamwise direction and this shifts the emergence of nonlinear effects, such as occlusion, downstream/upstream). Figure 3 shows snapshots of these computations, which are to be directly compared to panels $3 \mathrm{a}, 3 \mathrm{~b}$, and $3 \mathrm{c}$ in Camassa et al. (2014).

Agreement with the experimental snapshots is good. In particular, the wavelength of the four distinct surface waves at the tube outlet in panel $3 \mathrm{~b}$ is predicted accurately by our computations. Also, the number of gas bubbles (eleven) in panel 3c, which result from successive occlusion events, agrees with the experiment. Panel $3 \mathrm{c}$ and the supplementary movie M1 allow to discern the actual mechanics of occlusion. Surface waves in the upstream portion of the tube grow spatially until their crests reach the tube axis, whereupon individual bubbles of the gas core are pinched off. Our model accurately predicts the length scale of the pinch-off process in comparison with the experiment. We stress that panel $3 \mathrm{c}$ is a snapshot and that the occlusion point shifts up and down the tube intermittently as a result of the noisy wave spectrum imposed through the inlet condition. These results show that our model captures accurately the wave dynamics leading up to occlusion, and this is the focus of our current study. However, the physics of the liquid plugs resulting from occlusion is captured only very crudely by the numerical core radius limitation introduced in section 2.1. For example, the shape of the gas bubbles in panel $3 \mathrm{c}$ is quite different from that in the experiment and their length evolves as they travel downstream (see also supplementary movie M1). First results reported in appendix E suggest that these shortcomings can be alleviated with our improved WRIBL model. But, the exact shape of liquid plugs can never be fully captured in the context of lubrication theory, as the film surface slope diverges at their edges.

We focus now on panel 3a, where Re is closest to the experimental occlusion threshold. 

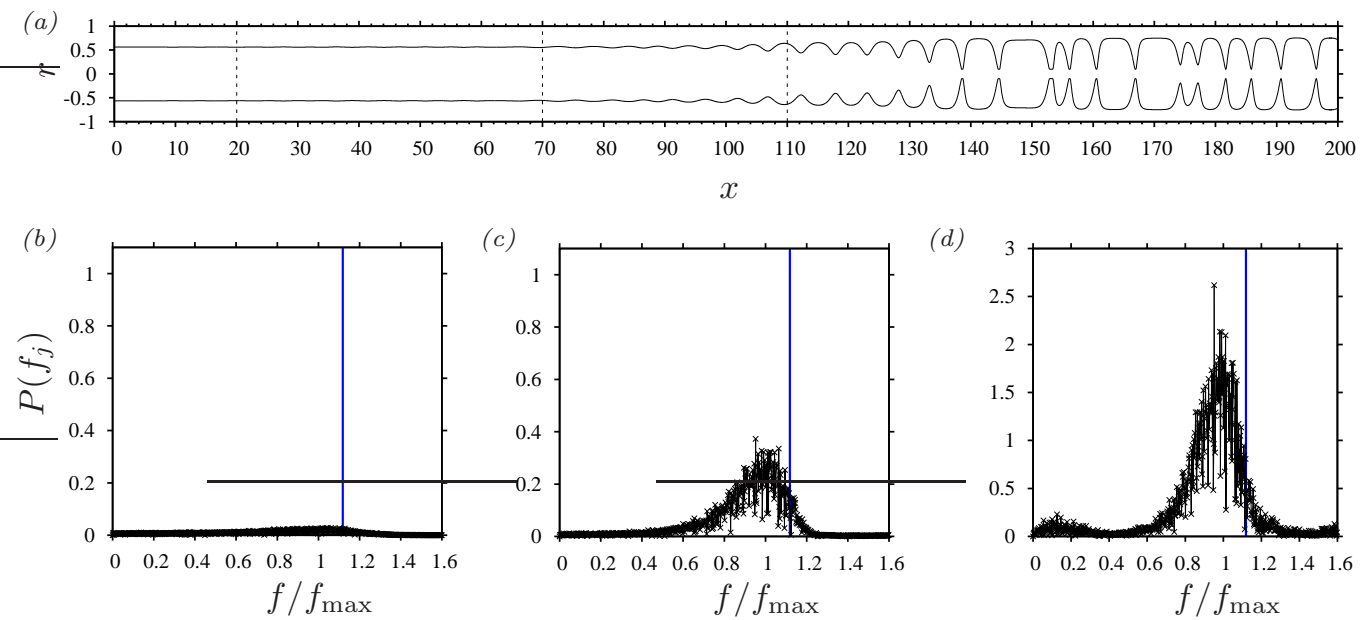

Figure 4. Nature of the prevailing surface waves that cause occlusion. Spatio-temporal computation reproducing the second version of the experiment in panel 3a of Camassa et al. (2014), which was performed in a longer $L^{\star}=1 \mathrm{~m}$ tube: case 1 in table $1, \operatorname{Re}=2.3 \times 10^{-4}$. (a) Snapshot of the computed film profile, showing individual surface waves that cause occlusion in the region $x \geqslant 130 ;(b-d)$ frequency spectra at different streamwise locations leading up to the occlusion region (marked by dashed lines in panel $a$ ): (b) $x=20$; (c) $x=70$; (d) $x=110$. Vertical blue lines at $f / f_{\max }=1.12$ mark limit of travelling wave solutions determined by numerical continuation (see panel $6 \mathrm{~b}$ ). $f_{\max }$ designates spatially most amplified frequency of linear waves.

For this case, Camassa et al. (2014) observed occlusion only after having switched to a longer $L^{\star}=1 \mathrm{~m}$ tube. Because of the small spatial growth rate, distinguishable surface waves emerged only very close to the outlet of the shorter $L^{\star}=400 \mathrm{~mm}$ tube, and their amplitude was insufficient to cause occlusion there. Our computation in panel 3a exhibits the same behaviour.

We have also reproduced numerically the experiment in the $L^{\star}=1 \mathrm{~m}$ tube and a snapshot of our computation is represented in panel $4 \mathrm{a}$. It confirms that occlusion does indeed occur, in the region $x \geqslant 130$. Occlusion results from unbounded spatial growth of the prevailing surface waves, and panels $4 \mathrm{~b}$ to $4 \mathrm{~d}$ show how the latter emerge from the noisy inlet condition. In these panels, we have represented the single-sided amplitude spectrum $P\left(f_{j}\right)$ of the discrete Fourier transform $F\left(f_{j}\right)$ applied to core radius time series $d\left(t_{k}\right)$ recorded at different streamwise positions $x$ (marked by dashed lines in panel $4 \mathrm{a}$ ), leading up to the occlusion region:

$$
P\left(f_{j}\right)=(2 / N) F\left(f_{j}\right) \forall j \leqslant N / 2+1, \quad F\left(f_{j}\right)=\sum_{k=0}^{N} d\left(t_{k}\right) \exp (i 2 \pi j / N k),
$$

where $N=1.8 \cdot 10^{6}$ is the number of (equidistant) sampling points $t_{k}$ with which we have recorded the time series $d\left(t_{k}\right)$ over a duration $T=9.1 \cdot 10^{4}$, and $f_{j}=(j / N) / T$ are the frequencies of the discrete spectrum. At the most downstream location (panel $4 \mathrm{~d}$ ), the spectrum is organized around the spatially most amplified frequency of linear waves $f_{\max }$ in the form of a quite narrow peak. This peak lies beyond the limit $f / f_{\max }=1.12$ for the existence of travelling-wave solutions at the considered Reynolds number $\operatorname{Re}=2.3 \times 10^{-4}$, which is highlighted by vertical blue lines in panels $4 \mathrm{~b}, 4 \mathrm{c}$, and $4 \mathrm{~d}$. This limit has been determined by numerical continuation of travelling-wave solutions, which we will discuss in the next subsection (it is marked by an asterisk in panel 5b). We will see that higher 
frequency (shorter) waves are safe from and lower frequency (longer) waves are prone to occlusion.

By way of numerical continuation, Camassa et al. (2014) were the first to show that the existence of travelling-wave solutions is bounded. By increasing Re at fixed wavelength, the authors found a limit point beyond which travelling-wave solutions cease to exist. They conjectured that the loss of such solutions signals occlusion in an actual experiment. However, the authors cautioned that further work was needed to verify this conjecture. This work was initiated by Camassa et al. (2016), but their model did not account for axial viscous diffusion. Our computations in figures 3 and 4 provide further validation, by taking into account this effect, which greatly improves agreement of our model predictions with the experiments of Camassa et al. (2014), as a result of the high viscosity of the working liquid. For example, when running the computation in panel 4a without axial viscous diffusion, we did not observe any occlusion over the entire experimental tube length (see figure 21 in appendix D), which is in qualitative contradiction to the experiments. Also, we will show in subsection 3.4 that neglecting axial viscous diffusion increases $\mathrm{Re}_{\max }$ by $74 \%$ for this liquid (panel 9a), moving it far beyond the experimental occlusion limit, as quantified in terms of Re.

Figure 4 proves that the occlusion of a narrow tube by a wavy falling liquid film is caused by surface waves that lie beyond the limit of travelling-wave solutions. Thus, occlusion can be characterized based on the existence span of such solutions and we proceed to this in the following subsection.

\subsection{Travelling waves: regimes of certain, conditional, and impossible occlusion}

The frequency of travelling waves determines their amplitude, which is well known for planar falling liquid films (Nosoko et al. 1996) and also holds in our cylindrical configuration. At a given value of Re, the smaller the wave frequency, the greater the wave amplitude. Consequently, low-frequency travelling waves are more prone to occlusion than high-frequency waves. This effect was demonstrated by Camassa et al. (2016) and Ding et al. (2019) with a first-order asymptotic model and a lubrication model, respectively. Using our model (2.5), we quantify it for the real liquids studied here (cases 1-4 in table 1), which require a more complicated modelling approach.

Panel 5a represents curves of travelling-wave solutions for case 1 in terms of the minimal core radius $d_{\min }$, as obtained by varying the liquid Reynolds number Re. On each curve, we have fixed the wave frequency $f$ to a certain fraction $f / f_{\max }$ of the spatially most amplified frequency $f_{\max }$, which we determine through the approach outlined in section 2.3. All curves display a limit point in terms of Re, beyond which there are no travellingwave solutions. The existence of such a limit point was discovered by Camassa et al. (2014), who imposed a constant wavelength in their continuation. This wavelength was too long compared to their experiments, whereas we see here that accounting for the correct wavelength/frequency is quite important. Indeed, the limit point of the curves in panel 5 a varies greatly with $f / f_{\max }$. The lower the wave frequency, the smaller the limit value of Re, the more danger of occlusion.

We now introduce the upper conservative occlusion bound $\mathrm{Re}_{\max }$, which corresponds to the limit point (LP) of the solid line representing the spatially most amplified travelling waves, i.e. $f / f_{\max }=1$. For $R e>R_{\max }$, the linearly most amplified surface waves, which are most likely to emerge in an experiment, do not possess travelling states and occlusion is certain to occur in a naturally evolving film. The experiment of Camassa et al. (2014), which we have reproduced numerically in figure 4, corresponds to this regime. Occlusion in this case occurs through scenario I, which we will discuss further in section 3.7 based on spatio-temporal computations. 

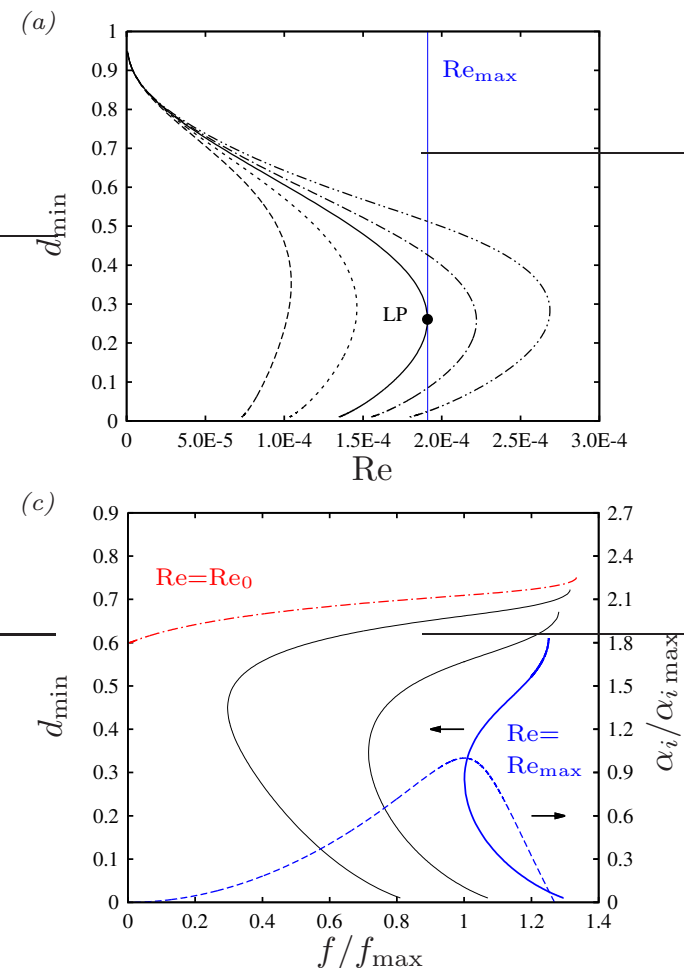
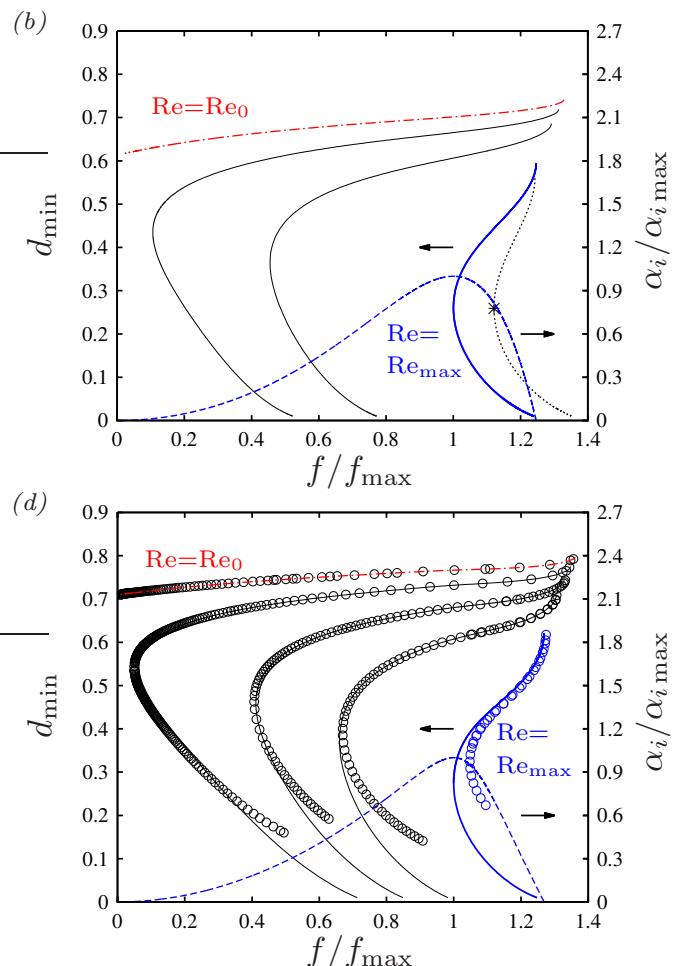

FiguRE 5. Limits of travelling-wave solutions obtained from numerical continuation with our model (2.5). Cases 1 (panels $a, b), 2$ (panel $c$ ), and 3 (panel $d$ ) in table 1. (a) Continuation of Re at wave frequency $f / f_{\max }=0.5$ (long dashes), 0.8 (short dashes), 1 (solid), 1.1 (dot-dashed), and 1.2 (dot-dot-dashed). Vertical blue line marks limit point (LP) for spatially most amplified linear waves, where $f=f_{\max }$ and $\operatorname{Re}=\operatorname{Re}_{\max } ;(b-d)$ frequency dependence at $\mathrm{Re}=$ const. Dashed blue: linear spatial growth rate $\alpha_{i}$ at $\mathrm{Re}=\mathrm{Re}_{\max }$ (right abscissae), other: travelling-wave solutions at $R e=$ const (left abscissae). (b) Case $1: \operatorname{Re}=\operatorname{Re}_{0}=6 \times 10^{-5}$ (dot-dashed red), $R e=7.5 \times 10^{-5}$, $1 \times 10^{-4}$ (thin solid), Re=Remax $=1.91 \times 10^{-4}$ (thick solid blue), and $\operatorname{Re}=2.3 \times 10^{-4}$ (dotted with asterisk); (c) case 2: $R e=R_{0}=0.012$ (dot-dashed red), $R e=0.016,0.025$ (thin solid), and $R e=R_{\max }=0.038$ (thick solid); (d) case 3: $R e=R_{0}=0.075$ (dot-dashed red), $R e=0.1,0.14,0.2$ (thin solid), and $\mathrm{Re}=\mathrm{Re}_{\max }=0.393$ (thick solid). Open circles correspond to data obtained with our own travelling-wave DNS (see section 2.2).

Panel 5b explains the meaning of the upper occlusion bound $\mathrm{Re}_{\max }$ in a different way, by illustrating the effect of the wave frequency $f$ at fixed Re. The dashed blue line represents the linear spatial growth rate $\alpha_{i}$ at $R e=R_{\max }$ (right abscissa), while all other curves represent travelling-wave solutions (left abscissa) at different Re. These curves all display a limit point in terms of $f$. The dotted line marked by an asterisk corresponds to the experiment of Camassa et al. (2014) numerically reproduced in figure 4 , where $\operatorname{Re}=2.3 \times 10^{-4}$. The thick blue solid line corresponds to the upper occlusion bound $\mathrm{Re}=\mathrm{Re}_{\max }=1.9 \times 10^{-4}$ and its limit point thus coincides with the maximum of the growth rate dispersion curve $\alpha_{i}(f)$. We stress that determining $\mathrm{Re}_{\max }$ requires simultaneously computing the most amplified wave frequency $f_{\max }$ in our numerical continuations, as described in section 2.3. This had not been attempted in the previous studies of Camassa et al. (2014) and Ding et al. (2019).

Reducing Re below $\mathrm{Re}_{\max }$ moves the limit point of travelling-wave solutions to lower values of $f$. Thus, the occlusion range is increasingly confined to (very long) waves of 
very low frequency, until it vanishes completely at $R e=\operatorname{Re}_{0}$ (red dot-dashed curve in panel 5b). This limit, the existence of which was discovered by Ding et al. (2019), constitutes a lower conservative occlusion bound. When $\operatorname{Re}<\mathrm{Re}_{0}$, occlusion is impossible, no matter how long the waves. In a real system, it is conceivable that wave coalescence events can produce local overshoots leading to occlusion even when $\operatorname{Re}<\mathrm{Re}_{0}$. However, we have not observed this in our computations of spatially evolving films (see panels $16 \mathrm{~b}$ and $17 \mathrm{c}$ ). The value $\mathrm{Re}_{0}$ being very low for all studied liquids, inertia is weak and significant overshoots are unlikely.

Based on the conservative occlusion bounds $R_{0}$ and $R_{\max }$, we delimit a region of conditional occlusion, $\operatorname{Re}_{0}<\operatorname{Re}<\operatorname{Re}_{\max }$ (between the red dot-dashed and thick solid blue lines in panel 5b), where occlusion is theoretically possible, for long enough waves, but does not necessarily occur in a real system. That depends on whether the required waves actually develop in a spatially evolving film. And, in particular, whether the tube is long enough to accommodate the nonlinear wave dynamics required to produce them. In section 3.7, we will demonstrate that occlusion in this regime occurs through scenario II, i.e. wave coarsening triggered by secondary instability, which shifts surface waves down the ascending branch of the dispersion curve toward increasingly low frequencies.

In panel $5 \mathrm{a}$, the limit point LP is a turning point, where two solution branches meet. As suggested by Camassa et al. (2014), we have checked their stability using transient periodic simulations with imposed $\Psi$ started from selected travelling-wave solutions. In these computations, numerical noise is responsible for perturbing the flow. A full-fledged formal stability analysis, such as the one performed by Camassa et al. (2016) based on the first-order model of Camassa et al. (2014), is outside the scope of our current study. We find that the lower solution branch is entirely unstable. As this branch does not connect to the flat-film primary flow $d_{\min }=d_{\max }=1$, the solutions thereon are thus highly unlikely in an experiment. Conversely, the upper branch is stable, at least w.r.t. periodic perturbations. However, wave trains of high-frequency travelling waves on falling liquid films are subject to secondary subharmonic and sideband instabilities that rely on wave interaction and lead to coalescence events (Liu \& Gollub 1993). We have found that this triggers occlusion scenario II, which we will demonstrate in §3.7. Finally, when starting a periodic transient computation from a flat-film initial condition beyond the limit of travelling-wave solutions, the outcome is indeed occlusion, which we have checked (and validated with transient DNS) in appendix A (see panels 19a and 19b).

Panels 5c (case 2), 5d (case 3), and figure 6 (case 4) report the occlusion bounds $\mathrm{Re}_{\max }$ and $\mathrm{Re}_{0}$ for the other three cases from table 1 . In panel $5 \mathrm{~d}$, we have represented with open circles additional results obtained with our own travelling-wave DNS (see section 2.2), evidencing gratifying agreement with our WRIBL model predictions. Overall tendencies in these graphs are the same as for case 1. For case 4 (low-viscosity silicone oil), where the Kapitza instability is relevant, there is an additional feature. In panel 6a, the continuation curves of $d_{\min }(\mathrm{Re}, f=$ const) at high frequencies, e.g. the dot-dot-dashed curve at $f / f_{\max }=1.2$, exhibit two lobes that are each associated with a limit point. Wave profiles corresponding to these highlighted points LP1 and LP2 are represented in panel 6c. We see that the main wave hump is preceded by a small precursory ripple. Such socalled capillary ripples can produce multiplicity of solutions in falling liquid films, when they are close enough to interact with the following wave hump (Kalliadasis et al. 2012). By contrast, the dashed curves in panel 6a, which pertain to low-frequency waves, each display only a single limit point (LP). Panels $6 \mathrm{~d}$ and $6 \mathrm{e}$ show the corresponding wave profiles at those points. We see in panels $6 \mathrm{c}, 6 \mathrm{~d}$, and $6 \mathrm{e}$ that lower wave frequencies are associated with longer wavelengths, which we have implicitly assumed up until now.

Finally, panel $6 \mathrm{f}$ represents the wave profile corresponding to the limit point (LP) 
(a)

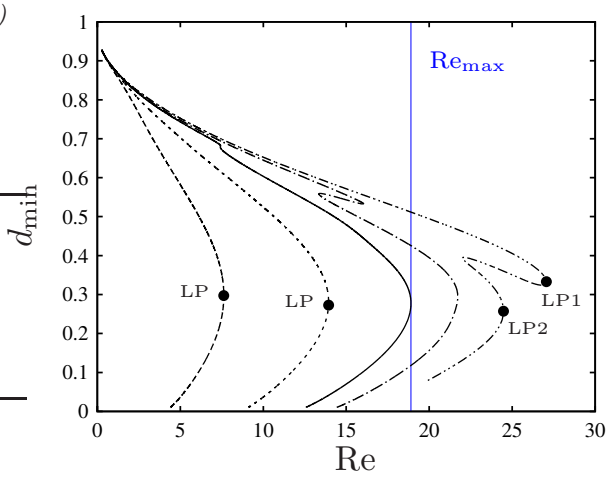

(b)

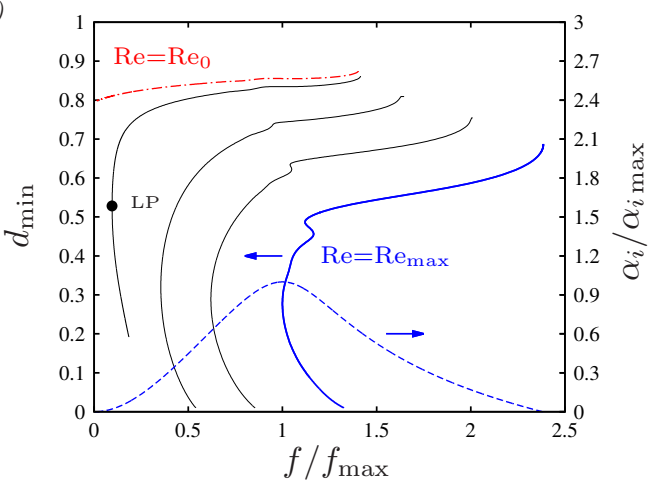

(c)

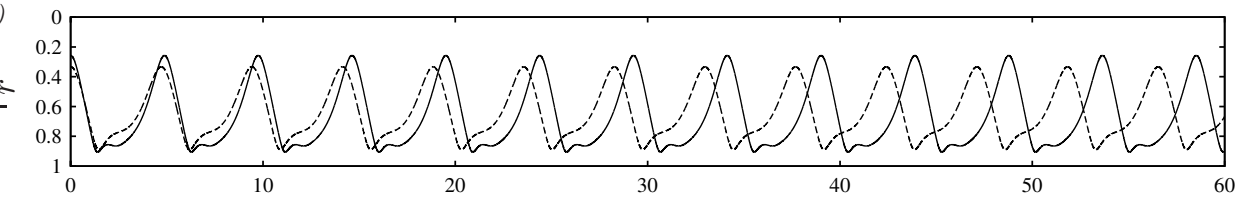

(d)

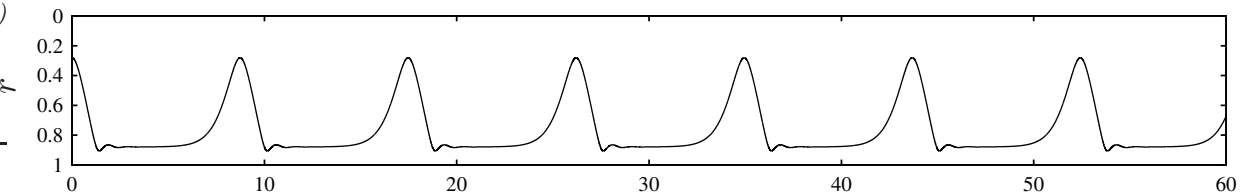

(e)

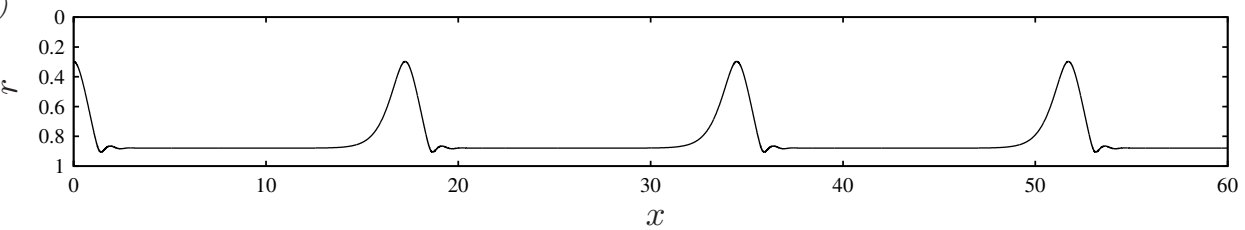

(f)

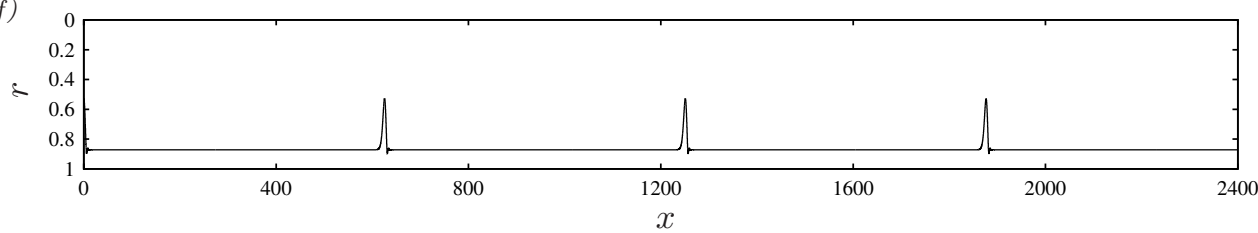

Figure 6. Limits of travelling-wave solutions for case 4 in table 1. (a) Continuation of Re at constant wave frequency $f / f_{\max }=0.5$ (long dashes), 0.8 (short dashes), 1 (solid), 1.1 (dot-dashed), and 1.2 (dot-dot-dashed); $(b)$ frequency dependence at $R e=$ const. Dashed: linear spatial growth rate $\alpha_{i}$ at $R e=\operatorname{Re}_{\max }=18.9$ (right abscissa); other: travelling-wave solutions (left abscissa) at $\operatorname{Re}=\operatorname{Re}_{0}=1.5$ (dot-dashed red), $\operatorname{Re}=2,5,10$ (thin solid), and $\operatorname{Re}=\operatorname{Re}_{\max }$ (thick blue); $(c-e)$ film profiles for LPs marked by filled circles in panel $a$; (c) $f=1.2 f_{\max }$. Solid: LP1; dashed: LP2; $(d) f=0.8 f_{\max } ;(e) f=0.5 f_{\max } ;(f)$ profile for marked LP in panel $b$.

marked by a filled circle on the $\mathrm{Re}=2$ curve in panel $6 \mathrm{~b}$. This curve lies in the region of conditional occlusion, $\operatorname{Re}_{0}<\operatorname{Re}<\operatorname{Re}_{\max }\left(\operatorname{Re}_{0}=1.5, \mathrm{Re}_{\max }=18.9\right)$. However, based on the wavelength of the waves in panel $6 \mathrm{f}$, which span about 600 times the tube radius, it becomes clear that quite particular conditions can be required to cause occlusion in this regime. This underlines the conservative nature of the lower occlusion bound $\mathrm{Re}_{0}$.

In summary, due to the frequency dependence of travelling-wave solutions demonstrated in panels $5 \mathrm{~b}, 5 \mathrm{c}, 5 \mathrm{~d}$, and $6 \mathrm{~b}$, three characteristic regimes can be delimited: (i) impossible occlusion, when $\operatorname{Re}<\operatorname{Re}_{0}$, (ii) conditional occlusion, when $\operatorname{Re}_{0}<\operatorname{Re}<\operatorname{Re}_{\max }$, 
(a)

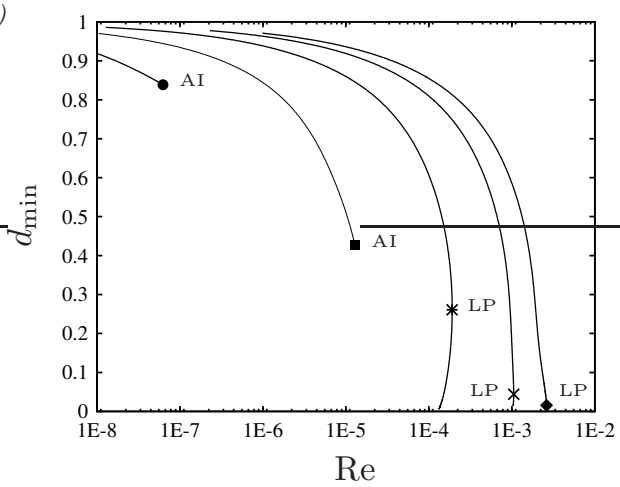

(b)

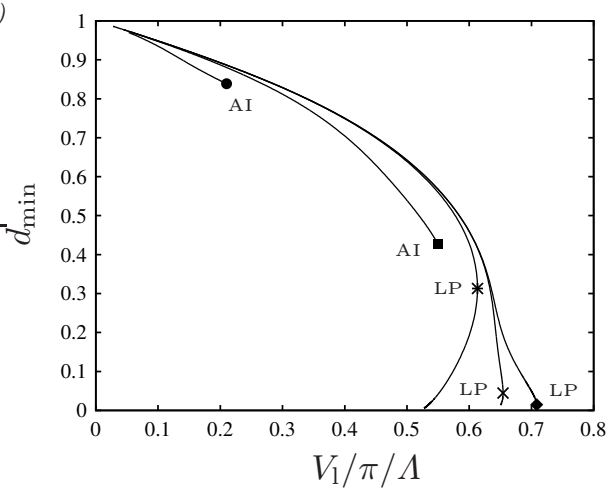

Figure 7. Delay of occlusion by increasing the Bond number $\mathrm{Bo}=\rho_{1} g R^{\star 2} / \sigma$ at constant Laplace number: $\mathrm{La}=\sigma \rho_{1} R^{\star} / \mu_{1}^{2}=6.27 \times 10^{-4}$ (case 1 ). Travelling-wave solutions at $f=f_{\max }$. Symbols mark onset of absolute linear instability (AI) or limit points (LP). The limit points correspond to the upper occlusion bound $\mathrm{Re}_{\max }$. Circles: $\mathrm{Bo}=0.1$; squares: $\mathrm{Bo}=1$; asterisks: $\mathrm{Bo}=11.1$ (case 1); crosses: $\mathrm{Bo}=50$; diamonds: $\mathrm{Bo}=100$. (a) Minimal core radius $d_{\min }$ versus liquid Reynolds number; (b) minimal core radius versus liquid holdup.

and (iii) certain occlusion, when $\operatorname{Re}>\mathrm{Re}_{\max }$. In section 3.7, we will characterize these regimes with spatio-temporal computations and demonstrate the associated occlusion scenarios I and II. On the other hand, the frequency dependence of travelling-wave solutions opens the possibility of using coherent inlet forcing to prevent occlusion. By forcing regular waves of sufficiently high frequency, occlusion can be avoided versus a noise-driven wave evolution. We demonstrate this idea in section 3.8 based on spatiotemporal computations. In the next sections, 3.3 to 3.6 , we first proceed to establish how different physical effects, i.e. gravity, axial viscous diffusion, inertia, and the core gas flow, affect the upper conservative occlusion bound $\mathrm{Re}_{\max }$.

\subsection{Role of gravity}

The upper occlusion bound $\mathrm{Re}_{\max }$ can be significantly delayed by increasing the relative strength of gravity versus capillarity. We have quantified this by varying the Bond number $\mathrm{Bo}=\rho_{1} g R^{\star 2} / \sigma$ over several orders of magnitude at constant Laplace number $\mathrm{La}=6.3 \times 10^{-4}$. This value of La corresponds to the high-viscosity silicone oil (case 1), where inertia is always negligible.

Panel 7a represents travelling-wave solutions for different values of Bo. On each curve, we have imposed the frequency $f_{\max }$ of the most amplified spatially growing linear waves. All curves exhibit a limit value for the liquid Reynolds number Re. For Bo $\ll 1$, where the Plateau-Rayleigh instability dominates, this limit is dictated by the onset of absolute instability (AI), as discussed in panels $2 \mathrm{c}$ and $2 \mathrm{~d}$. Beyond this point, prevailing waves are no longer characterized by the most-amplified spatially growing linear waves and $f_{\max }$ is no longer defined. The solution curves stop abruptly. Occlusion in this regime is caused by absolute instability of the primary flow. This regime is not the focus of our study.

We focus on $\mathrm{Bo} \geqslant 1$, for which the solution curves in panel $7 \mathrm{a}$ are bounded by a limit point (LP), as discussed in panel 5a. This limit point marks the upper occlusion bound $\mathrm{Re}_{\max }$, which increases significantly with increasing Bo, while the minimal core radius $d_{\min }$ at LP significantly diminishes. The first observation is in line with the experiments of Dao \& Balakotaiah (2000), who reported Re at the experimental occlusion onset for different liquids and tube radii but had no access to the wave dynamics. The authors also 
(a)

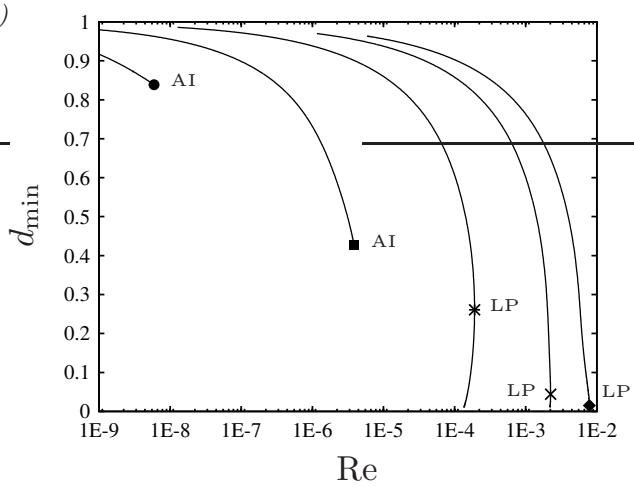

(c)

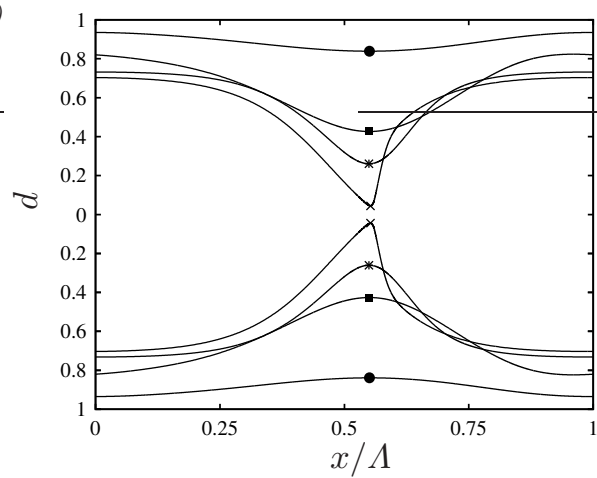

(b)

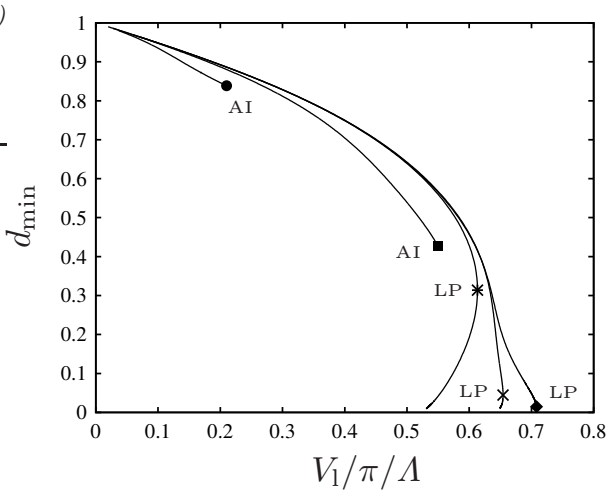

(d)

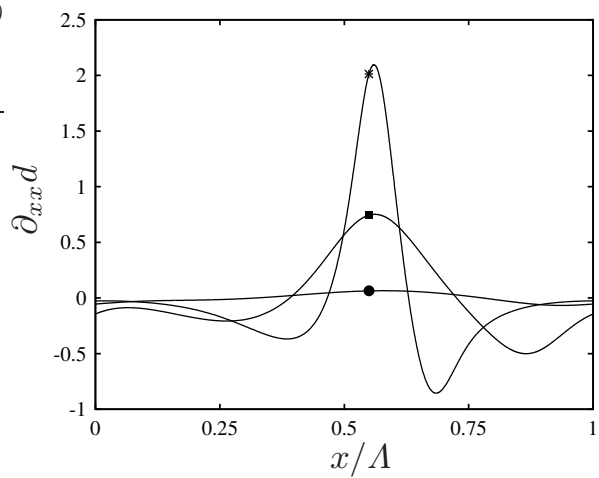

FiguRE 8. Delay of occlusion by increasing the Bond number Bo at constant Kapitza number: $\mathrm{Ka}=\sigma \rho_{1}^{-1} g^{-1 / 3} \mu_{1}^{-4 / 3}=3.29 \times 10^{-4}$ (case 1 ). This amounts to increasing the tube radius $R^{\star}$ while maintaining constant fluid properties. See figure 7 for symbol attributions. (a) Minimal core radius $d_{\text {min }}$ versus liquid Reynolds number; (b) minimal core radius versus liquid holdup; (c) surface profiles at AI and LP; (d) corresponding profiles of axial curvature $\partial_{x x} d$.

outlined an approach for predicting occlusion based on travelling waves, which relied on the conjecture that the wave height always reaches the tube radius as Re is increased toward the occlusion limit. Based on our results in panel 7a, this conjecture only holds when Bo is extremely large. Otherwise, travelling-wave solutions are lost well before their height attains the tube radius.

In panel $7 \mathrm{~b}$, we have replotted our results from panel $7 \mathrm{a}$ in terms of the liquid hold-up $V_{1} / \pi / \Lambda$. We see that the maximally achievable liquid hold-up increases significantly with Bo. This again is in line with the experiments of Dao \& Balakotaiah (2000), who had observed that the mean film thickness $\bar{h}$ at occlusion is significantly greater than in the gravity-free Plateau-Rayleigh configuration (Gauglitz 1988).

The variation of Bo in figure 7 amounts to varying the gravitational acceleration for constant liquid and tube radius. Of course, it is either very difficult (low Bo range, microgravity experiments) or impossible (high Bo range) to realize the displayed range of variation $0.1 \leqslant$ Bo $\leqslant 100$ practically. A more practicable way to quantify the desired effect is to increase the tube radius $R$ for a constant liquid. This amounts to increasing Bo at constant $\mathrm{Ka}=\sigma \rho_{1}^{-1} g^{-1 / 3} \mu_{1}^{-4 / 3}$. We have performed such computations for $\mathrm{Ka}=3.3 \times 10^{-3}$, corresponding to the high-viscosity silicone oil (case 1). Results are represented in panels $8 \mathrm{a}$ and $8 \mathrm{~b}$, and these display the same overall behaviour as figure 7 .

The delay of $\mathrm{Re}_{\max }$ results from a gravity-induced streamwise distortion of surface waves, which favours variations in (stabilizing) axial interface curvature over variations in 
(destabilizing) azimuthal curvature. Panel 8c illustrates this distortion, which is marked by a compression of the leading wave front and an elongation of the trailing front. The different surface profiles represented correspond to the absolute instability thresholds (AI) and limit points (LP) highlighted in panel 8a. Panel 8d represents corresponding profiles of the axial curvature $\partial_{x x} d$. The variation in (stabilizing) axial curvature $\partial_{x x} d$ around the wave crest (marked by a symbol) significantly increases in magnitude with increasing Bo. This allows to counter a larger variation in (destabilizing) azimuthal curvature, and, thus, to saturate waves of greater amplitude.

The saturation mechanism was identified by Frenkel et al. (1987) for pressure-driven core annular flows, where it is due to a viscous interfacial shear stress, and demonstrated experimentally for falling liquid films on cylindrical fibres by Quéré (1990). In both studies, the saturation took hold in the weakly-nonlinear regime, limiting waves to a very small amplitude. Thus, it was perceived as suppressing macroscopically-visible waves altogether. In our case, the mechanism takes hold in the strongly-nonlinear regime, allowing to saturate travelling waves of greater amplitude as Bo is increased.

The role of gravity is kinematic, i.e. it makes initial surface elevations travel faster than surface depressions (Dietze 2016), and this produces the distortion of wave fronts observed in panel 8c. We point out that this effect was precluded in Jensen (2000), where surface waves were constructed in the form of perfectly symmetrical unduloids.

\subsection{Role of axial viscous diffusion}

Axial viscous diffusion, emanating from the $\partial_{x x} u$ term in (2.1), is represented in our model (2.5) through the terms with coefficients $J_{j}, K_{j}, L_{j}$, and $M_{j}$. These terms appear when the underlying long-wave expansion is performed up to second order. This was not done in the previous modelling works of Trifonov (1992), Camassa et al. $(2014,2017)$, Zhou et al. (2016), Liu \& Ding (2017), and Ding et al. (2019). So, the question is whether retaining these terms is worth the effort. We have checked this in panels $9 \mathrm{a}, 9 \mathrm{~b}$, and $9 \mathrm{~d}$, representing travelling-wave solutions for cases 1,3 , and 4 . The three cases span a wide range of the capillary number $\mathrm{Ca}=\mu_{1} \mathcal{U} / \sigma$, which relates viscous to capillary stresses, and which quantifies the relevance of axial viscous diffusion.

In figure 9, solid lines correspond to the full model (2.5) and dot-dashed lines to the limit $J_{i}=K_{i}=L_{i}=M_{i}=0$. Further, we distinguish two types of continuations. On the blue curves, which contain no symbols, we have fixed the wavelength $\Lambda=12.56$. On the black curves with symbols, we have imposed the spatially most amplified frequency of linear waves $f=f_{\max }$. In that case, axial viscous diffusion may affect the solution both nonlinearly and linearly (via wave selection), but the latter effect is negligible for the cases considered here. By comparing the solid and dashed curves in panels $9 \mathrm{a}, 9 \mathrm{~b}$, and $9 \mathrm{~d}$, we see that axial viscous diffusion precipitates occlusion in general and $\mathrm{Re}_{\max }$ in particular and this effect increases with Ca.

For the high-viscosity silicone oil (panel 9a, case 1: $\mathrm{Ca}=0.37$ ), accounting for axial viscous diffusion greatly reduces $\mathrm{Re}_{\max }$, i.e. from $\mathrm{Re}_{\max }=3.3 \mathrm{E}-4$ to $\mathrm{Re}_{\max }=1.9 \mathrm{E}-4$ (compare dot-dashed and solid black lines with symbols). It is insightful to compare these values to the experimental occlusion bound, which was observed by Camassa et al. (2014) between $\mathrm{Re}=1.6 \mathrm{E}-4$ and $\mathrm{Re}=2.3 \mathrm{E}-4$ for their $1 \mathrm{~m}$ long tube. Linear interpolation based on their table 1 and figure 11 yields a value of $R e=2.2 \mathrm{E}-4$, which is not much greater than our $\mathrm{Re}_{\max }$ prediction with $\left(\mathrm{Re}_{\max }=1.9 \mathrm{E}-4\right)$ but much smaller than our prediction without axial viscous diffusion $\left(\mathrm{Re}_{\max }=3.3 \mathrm{E}-4\right)$. Interestingly, when basing the comparison on the mass-equivalent core radius $d_{m}=\left(L^{-1} \int_{0}^{L} d^{2} \mathrm{~d} x\right)^{1 / 2}$, which was also measured by Camassa et al. (2014), our two predictions $\left(d_{m}^{\star}=0.31 \mathrm{~cm}\right.$ versus $\left.d_{m}^{\star}=0.25 \mathrm{~cm}\right)$ lie equally close to the 
(a)

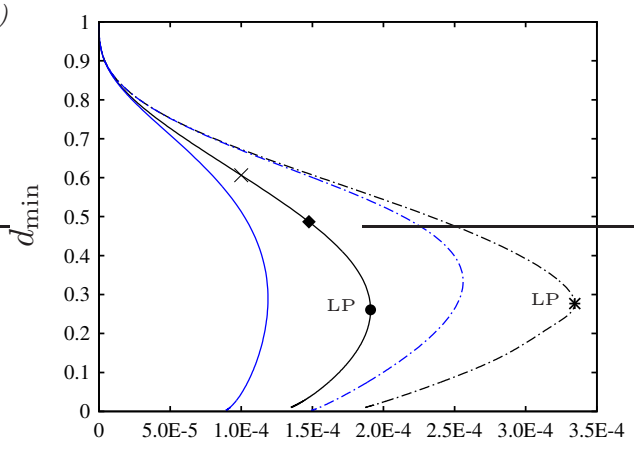

Re

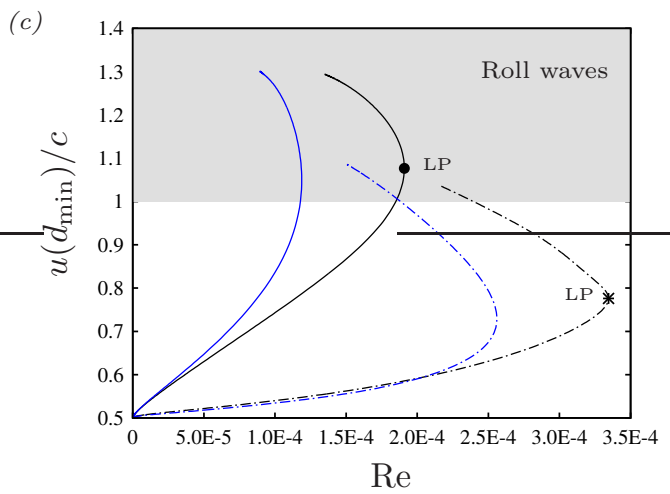

(b)

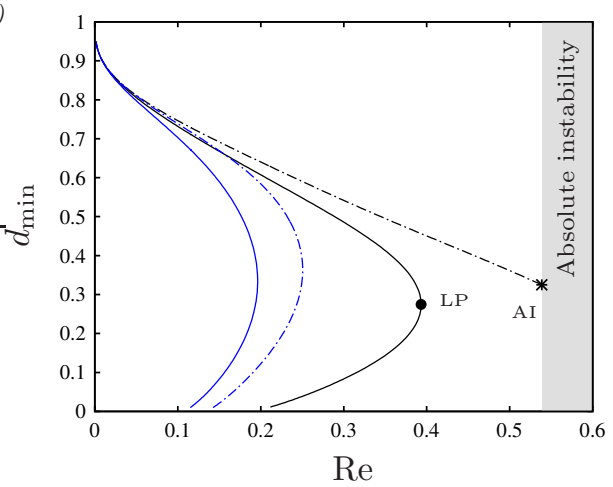

(d)

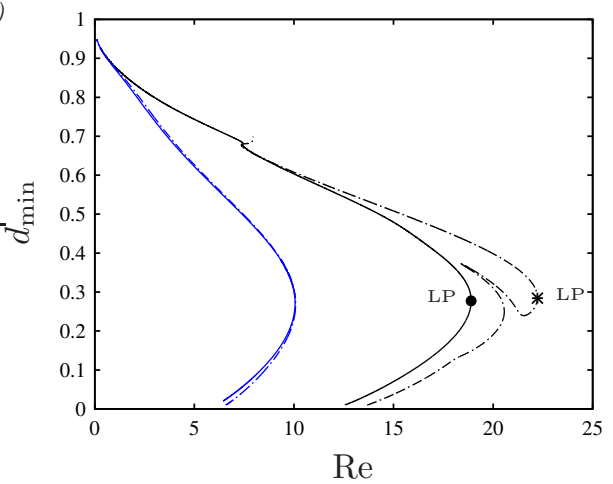

Figure 9. Precipitation of occlusion due to axial viscous diffusion. Cases 1, 2, and 3 . Circles/asterisks mark limit points (LP) and absolute instability onset (AI). Solid lines: full model (2.5); dot-dashed lines: no axial viscous diffusion, $J_{j}=K_{j}=L_{j}=M_{j}=0$ in (2.5) and (2.11); black curves with symbols: $f=f_{\max }$; blue lines without symbols: $\Lambda=12.56$. The capillary number Ca scales the observed effect. (a,c) Case 1: $\mathrm{Ca}=0.37$. Cross and diamond mark points used in figure 10. Panel $c$ represents ratio of interfacial fluid velocity $u\left(d_{\min }\right)$ to wave speed $c$. Grey region marks existence of roll waves $u\left(d_{\min }\right)>c$; (b) case 3 : $\mathrm{Ca}=0.028$. In the grey region, the primary flow is absolutely unstable $(\mathrm{AI}) ;(d)$ case 4 : $\mathrm{Ca}=0.012$.

experimental value $d_{m}^{\star}=0.28 \mathrm{~cm}$. This explains why Camassa et al. $(2014,2016)$ observed good agreement in terms of this parameter, although their model did not account for axial viscous diffusion. Finally, we point out that the corresponding values of the primary-flow core radius $d_{0}$, which is more representative of the flat film portion in the upper part of the experimental tube, are $d_{0}^{\star}=0.29 \mathrm{~cm}$ with and $d_{0}^{\star}=0.24 \mathrm{~cm}$ without axial viscous diffusion.

For case 3 (panel 9b, $\mathrm{Ca}=0.028$ ), which corresponds to experimental run 13 in Dao \& Balakotaiah (2000), axial viscous diffusion dictates even the nature of the occlusion mechanism. For the continuation at $f=f_{\max }$ (black lines with symbols in panel $9 \mathrm{~b}$ ), occlusion is caused by absolute linear instability (AI) when axial viscous diffusion is neglected (dot-dashed line), and by nonlinear loss of travelling-wave solutions (LP) when it is taken into account (solid line).

For the lowest $\mathrm{Ca}$ (panel 9d, case 4: $\mathrm{Ca}=0.012$ ), neglecting axial viscous diffusion does not meaningfully affect $\mathrm{Re}_{\max }$. It is however responsible for the formation of a second lobe on the black dot-dashed curve marked by an asterisk, which results from a change in the number of precursory ripples, as discussed w.r.t. panels $6 \mathrm{a}$ and $6 \mathrm{c}$.

The nonlinear mechanism by which axial viscous diffusion precipitates occlusion is 
(a)

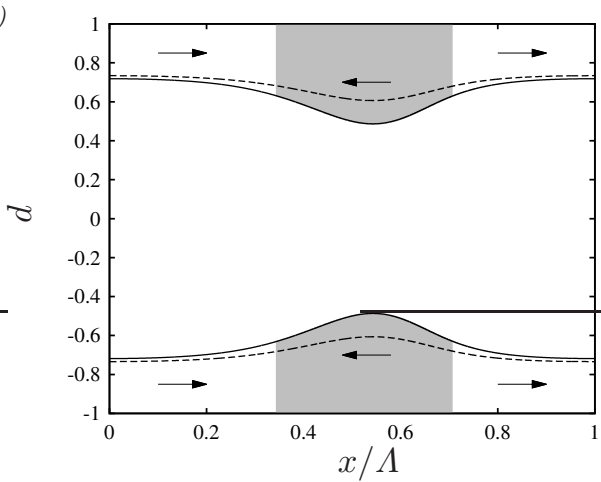

(b)

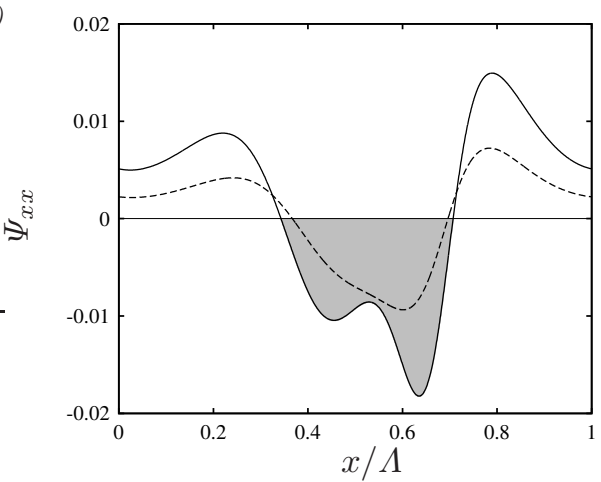

FiguRE 10. Mechanism delaying occlusion due to axial viscous diffusion. Data correspond to the two solution points marked by a cross and a diamond in panel 9a. Solid lines: diamond in panel 9a, $R e=1.47 \times 10^{-4}$; dashed lines: cross in in panel $9 \mathrm{a}, \mathrm{Re}=1.0 \times 10^{-4}$. (a) Surface profiles with zones of negative (grey) and positive (white) $\Psi_{x x}$; (b) normalized differential normal axial viscous stress $\Psi_{x x}$ according to (3.2). Axial normal viscous stresses counteract gravity in the wave fronts, tending to steepen the trailing and flatten the leading wave front.

illustrated in figure 10. Panel 10a represents surface profiles corresponding to the two points marked by a diamond and a cross in panel 9a. The solid profile in panel 10a corresponds to the point marked by a diamond in panel 9a and the dashed profile to the point marked by a cross. In panel 10b, we have plotted corresponding profiles of the normalized axial normal viscous force $\Psi_{x x}$ :

$$
\Psi_{x x}=\frac{\operatorname{Fr}^{2}}{\operatorname{Re}} \frac{\partial_{x} F_{x x}}{\pi\left(1-d^{2}\right)}, \quad F_{x x}=2 \pi \int_{d}^{1} \partial_{x} u r \mathrm{~d} r,
$$

where $F_{x x}$ is the (dimensionless) axial viscous force acting on the cross section of the liquid film. Its differential $\mathrm{d} F_{x x}=\partial_{x} F_{x x} \mathrm{~d} x$ yields the resulting axial normal viscous force acting on a slice of liquid film. In (3.2), we have normalized $\mathrm{d} F_{x x}$ with the differential gravitational force to obtain $\Psi_{x x}$.

Grey and white areas underneath the solid curves in panels 10a and 10b identify regions where the axial normal viscous force is directed counter to $\left(\Psi_{x x}<0\right)$ or in the direction of $\left(\Psi_{x x}>0\right)$ gravity. Based on this, the arrows in panel 10a illustrate the resulting action of axial normal viscous stresses. This action tends to steepen the trailing front of the surface wave (where the residual film and the wave hump are pushed toward one another) and elongate the leading wave front (where residual film and wave hump are pulled away from one another). It counters the gravity-induced compression of the leading wave front and elongation of the trailing wave front and thus weakens the saturation mechanism discussed in section 3.3. The strength of the effect increases with increasing Reynolds number, as evidenced by comparing the two profiles in panel 10b and this explains why the solid and dot-dashed lines in panel 9a diverge with increasing Re.

Axial viscous diffusion also greatly affects the wave speed and the streamline pattern in the wave-fixed reference frame. In panel 9c, we have replotted the continuation curves from panel $9 \mathrm{a}$ in terms of the fluid velocity at the wave crest $u\left(d_{\min }\right)$ normalized by the wave celerity $c$. When $u\left(d_{\min }\right)>c$, a moving-frame vortex develops in the wave hump. Such waves are known as roll waves and the grey area in panel 9c delimits their existence. The limit point on the solid black line (filled circle) lies within this region, while the limit point on the dot-dashed line (asterisk) does not. This is illustrated by the corresponding streamline patterns in figure 11. Also, we see that the roll wave in panel 11a conveys with 


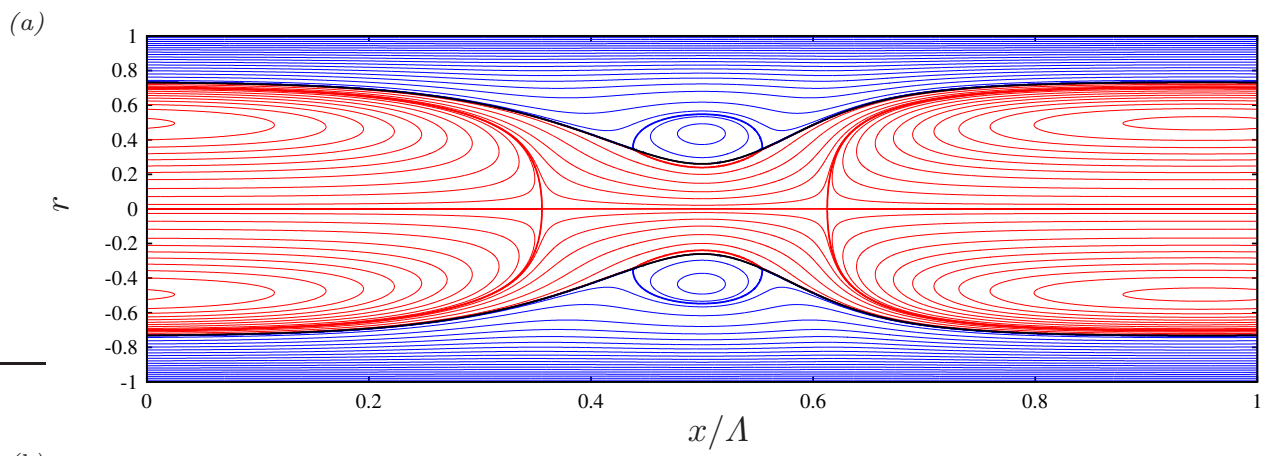

(b)

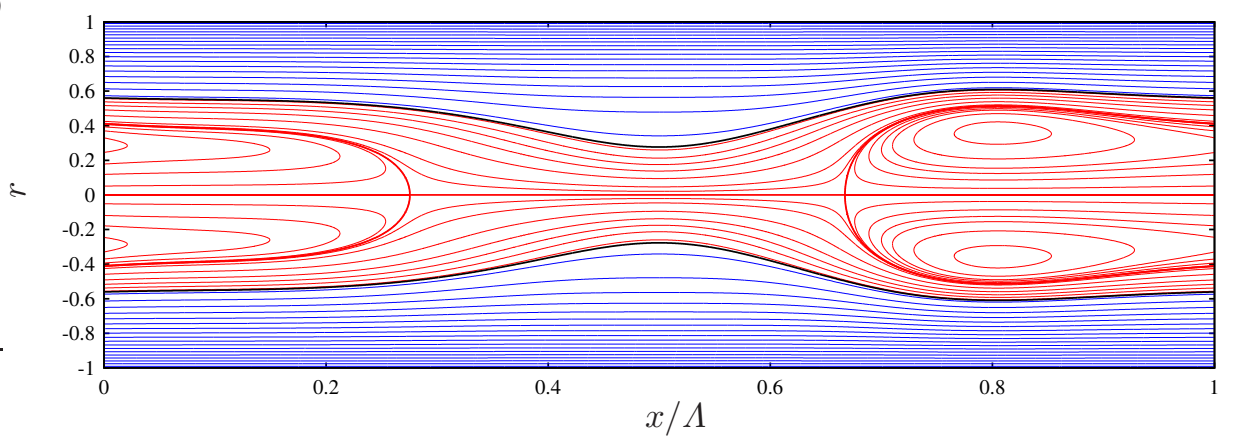

FIgURE 11. Streamlines in the wave-fixed reference frame for the marked limit points (LP) in panel 9c. Blue lines correspond to liquid film and red lines to core gas flow. (a) Full model (2.5): filled circle in panel 9c. The wave hump contains a vortex; (b) no axial viscous diffusion, i.e. $J_{j}=K_{j}=L_{j}=M_{j}=0$ in (2.5): asterisk in panel 9c. No vortex is predicted in the wave hump.

it a large gas bubble containing a toroidal vortex. This particular flow pattern results from the aerostatic pressure difference imposed here, i.e. $\Psi=1$. Other gas flow scenarios will be discussed in section 3.6. Finally, we point out that the lower branches in panel 9 are the stable ones, and that only the full model, which accounts for axial viscous diffusion, predicts roll wave solutions on these branches.

\subsection{Role of inertia}

Inertia constitutes the growth mechanism of the Kapitza instability, which is responsible for the formation of surface waves on planar falling liquid films, and which becomes relevant in our current problem when Re is sufficiently large. This is the case for the lowviscosity silicone oil film (case 4). In figure 12, we have represented nonlinear travellingwave solutions (panel 12a) and dispersion curves of the linear spatial growth rate $\alpha_{i}$ (panel 12b) for this case. Solid curves were obtained with our full model (2.5), and dashed curves by neglecting inertia, i.e. by setting $S_{i}=F_{i j}=G_{i j}=0$ in (2.5) and (2.11).

In panel $12 \mathrm{a}$, we confront two types of travelling-wave continuations. On the blue lines without symbols, we have fixed the wavelength $\Lambda=12.56$. In this case, the effect of inertia is purely nonlinear. We see that this effect significantly advances the limit point of the solid blue versus the dashed blue curve (by approximately 20\%). Inertia increases the cumulated strength of the destabilizing mechanisms (Plateau-Rayleigh and Kapitza instabilities) versus the stabilizing ones (capillarity and viscous dissipation). This limits the ability to saturate nonlinear travelling waves to smaller amplitudes, i.e. lower Re.

On the black lines with symbols, we have imposed $f=f_{\max }$. Here, the effect of inertia 
(a)

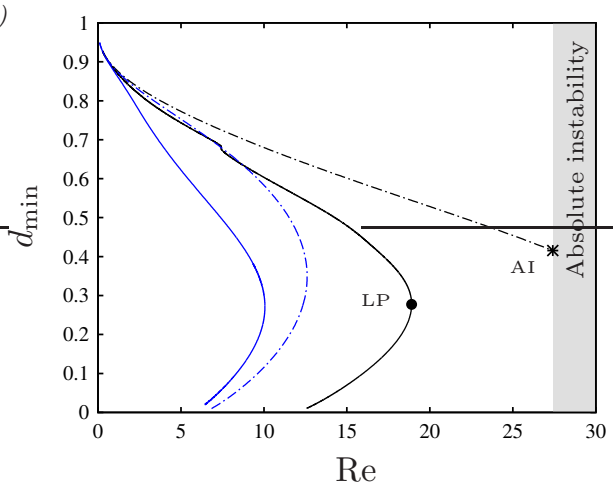

(b)

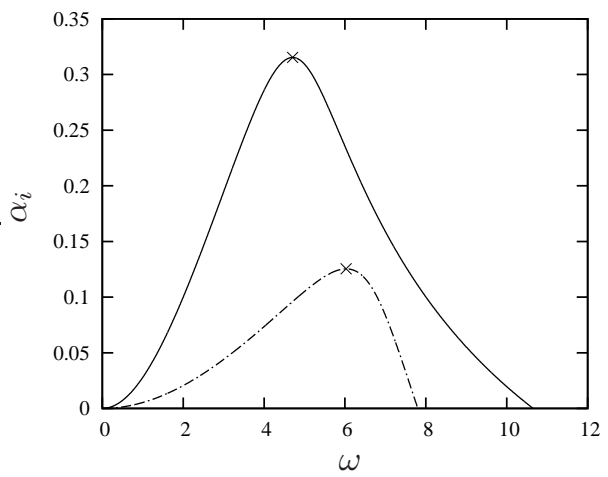

Figure 12. Precipitation of occlusion due to inertia. Case 4: low-viscosity silicone oil, Bo=0.993. Solid lines: full model (2.5); dot-dashed lines: no inertia, $S_{i}=F_{i j}=G_{i j}=0$ in (2.5) and (2.11). (a) Nonlinear travelling-wave solutions. Black lines with symbols: $f=f_{\max }$; blue lines without symbols: $\Lambda=12.56$; (b) dispersion curves of linear spatial growth rate $\alpha_{i}$ : $\operatorname{Re}=15.4$.

is even stronger, as it additionally affects linear wave selection by determining the spatially most amplified wave frequency $f_{\max }$. The linear dispersion curves in panel $12 \mathrm{~b}$, where $R e=15.4$, illustrate this effect. When inertia is taken into account (solid curve), $\omega_{\max }=2 \pi f_{\max }$ moves to smaller frequencies, which we have shown to precipitate the loss of travelling-wave solutions (panel 6a in §3.2). Comparing the solid and dashed lines in panel 12a, we see that inertia, similar to axial viscous diffusion, also dictates the nature of the occlusion mechanism. When it is taken into account, occlusion results from the loss of travelling-wave solutions at the limit point (LP) marking the upper occlusion bound $\mathrm{Re}_{\max }$. When it is neglected, occlusion occurs to to absolute instability (AI).

In addition to shifting $\mathrm{Re}_{\max }$, inertia also changes the nature of the occlusion bound in panel 12a. If inertia is neglected, $\mathrm{Re}_{\max }$ is dictated by absolute instability (AI, asterisk), otherwise, it is dictated by a nonlinear turning point (LP, filled circle). This qualitative change was also observed for the role of axial viscous diffusion in figure 9 .

In the case of the classical Plateau-Rayleigh configuration, Bo $\rightarrow 0$, inertia only affects the dynamics of the film's evolution (Dietze \& Ruyer-Quil 2015) but not the occlusion limit itself, because the latter is determined by a static equilibrium state (Everett \& Haynes 1972).

\subsection{Role of core gas flow}

The gas in the core of the cylindrical tube can affect the liquid film falling down its inner wall in two ways: (i) through the normal and tangential interfacial viscous stresses $\tau_{\mathrm{g}}^{\mathrm{n}}$ and $\tau_{\mathrm{g}}^{\mathrm{t}}$, which are scaled by $\Pi_{\mu}$ in (2.3), and (ii) through the interfacial gas pressure $p_{\mathrm{g}}$, which is scaled by $\Pi_{\rho}$ in (2.3). Up until now, we have assumed that the gas is subject to an aerostatic pressure difference, $\Psi=1$, meaning that gravity is fully compensated in the gas. In that case, the gas flow is enslaved to the hydrodynamics of the liquid film and both the above effects are negligible. Nonetheless, this one-way coupling produces intricate flow structures in the gas, as has been suggested by panel 11a and will be discussed further based on panel 14a.

Conversely, when the gas flows counter-currently at a Reynolds number $R_{\mathrm{g}}$ of sufficient magnitude, it does affect the hydrodynamics of the liquid film, and this two-way coupling can significantly precipitate the upper occlusion bound $\mathrm{Re}_{\max }$. We show this in figure 13 based on case 4 , which produces surface waves of sufficient amplitude to significantly 
(a)

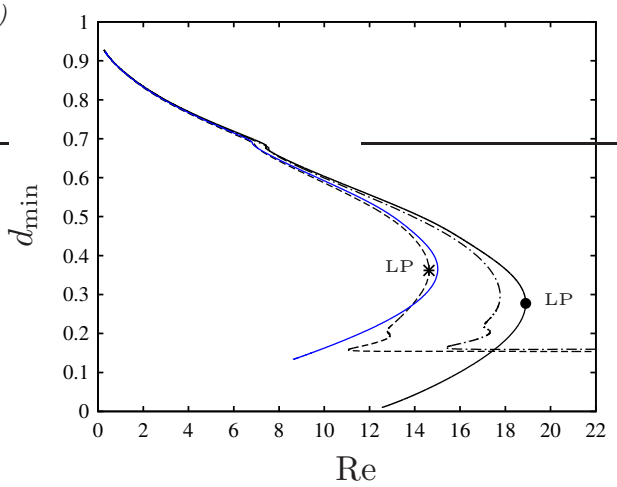

(c)

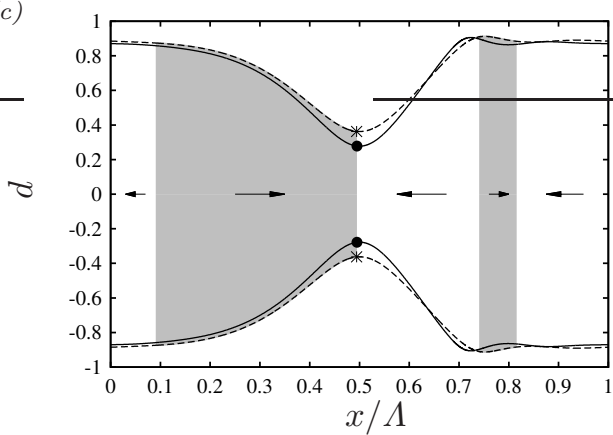

(b)

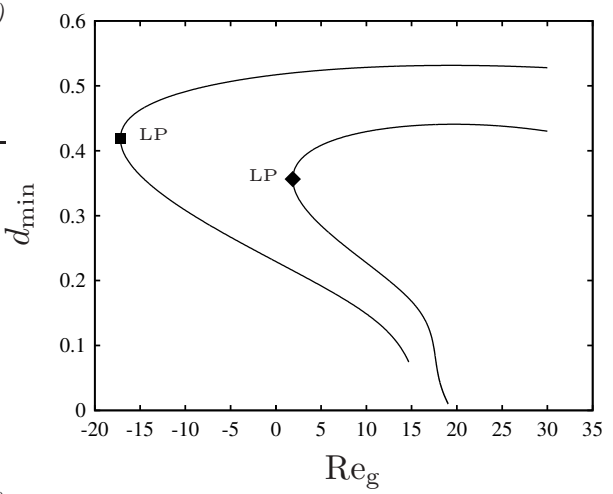

(d)

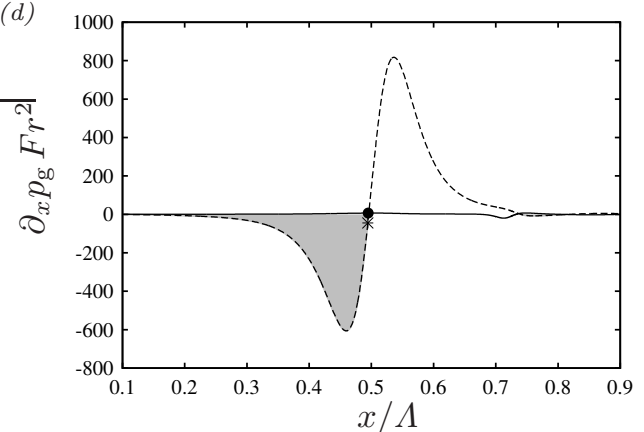

Figure 13. Precipitation of occlusion due to a counter-current gas flow. Case 4. (a) Travelling-wave continuation at $f=f_{\max }$. Solid: aerostatic pressure drop, $\Psi=1$; dashed: counter-current gas flow, $\operatorname{Re}_{\mathrm{g}}=-17.27$; solid blue line without symbol: $\Pi_{\mu}=0$ in $(2.5), \operatorname{Re}_{\mathrm{g}}=-17.27$; dot-dashed: $\Pi_{\rho}=0$ in $(2.5), R_{\mathrm{g}}=-17.27$; (b) continuation at $V_{1}=$ const and $f=f_{\max }$. Square: $V_{1} / \pi / R^{3}=2.435$; diamond: $V_{1} / \pi / R^{3}=2.770 ;$ (c) wave profiles corresponding to the asterisk and filled circle in panel $a ;(d)$ corresponding profiles of gas pressure gradient $\partial_{x} p_{\mathrm{g}}$ according to $(2.5 c)$ (symbols mark position of wave maximum). Grey/white zones between the dashed profiles in panels $c, d$ demarcate regions of negative/positive $\partial_{x} p_{\mathrm{g}}$. Arrows in panel $c$ indicate action of gas pressure gradient on the liquid film.

constrict the core gas flow. Panel 13a represents travelling-wave solutions obtained by continuing Re at $f=f_{\max }$ for different gas flow configurations. The solid black curve with a filled circle at the limit point corresponds to $\Psi=1$, and the dashed curve to a countercurrent gas flow with $R_{g}=-17.27$. Comparing these two curves, we see that $R_{\max }$ is significantly precipitated due to the counter-current gas flow (by roughly $25 \%$ ). We have checked that the gas flow does not meaningfully affect the most amplified frequency $f_{\max }$, and thus this precipitation is a nonlinear effect. The dashed curve also displays two qualitative changes. First, its lower branch turns forward at a second, lower, limit point, producing travelling-wave solutions of constant amplitude that exist far beyond $\mathrm{Re}_{\max }$. However, as we show in appendix C, these solutions are inaccessible in a real system. Thus, $\mathrm{Re}_{\max }$ remains a representative occlusion bound also in the counter-current configuration. Second, solutions on the portion of the curve between the asterisk and the new limit point are stable, in contrast to those on the lower branch of the solid black curve, where $\Psi=1$.

Panel 13b illustrates the effect of the gas flow in another way. Here, $R_{g}$ is varied at a fixed liquid volume $V_{1}$, while maintaining $f=f_{\max }$. Such a scenario may be relevant for 
(a)

(b)
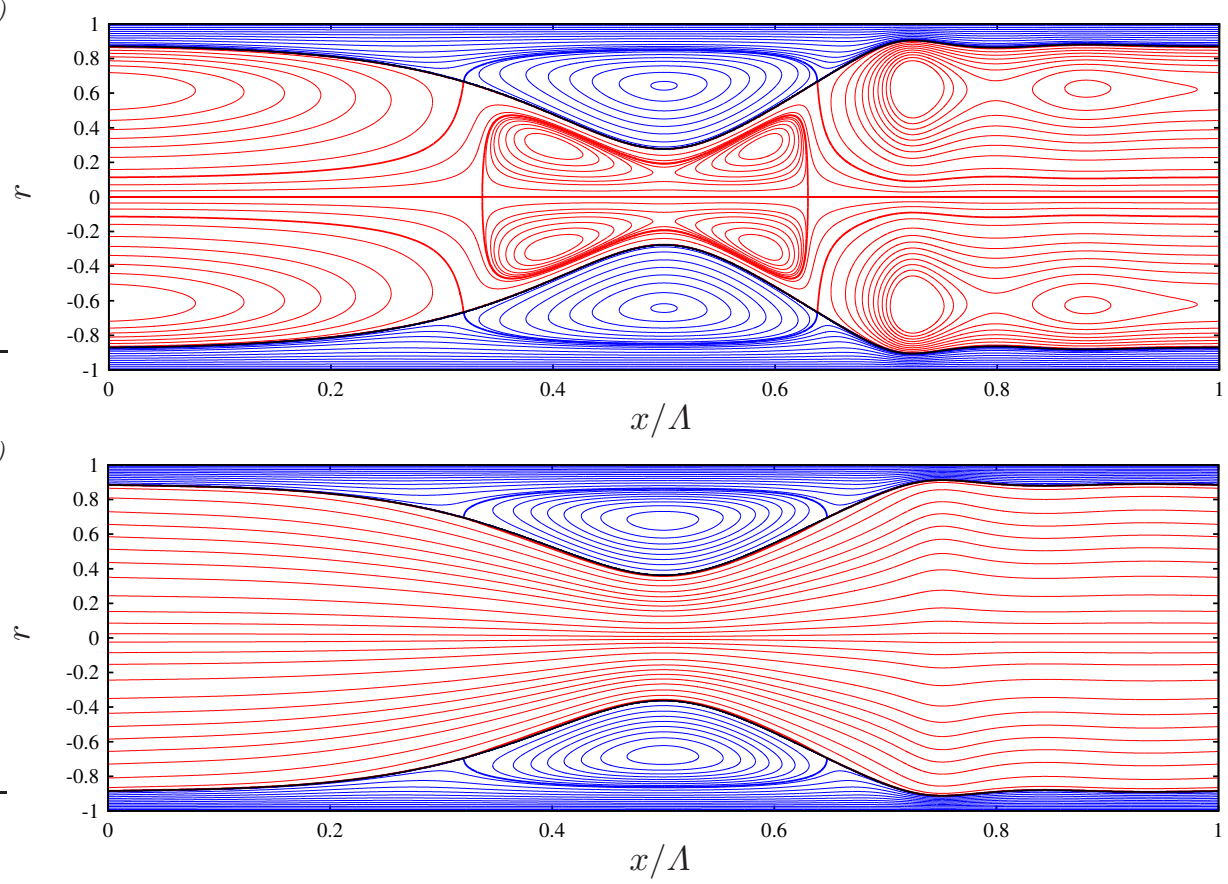

Figure 14. Streamlines in the wave-fixed reference frame corresponding to the marked limit points (LP) in panel 13a. Within the film, liquid below the vortex flows from right to left. (a) Aerostatic gas pressure drop (filled circle in panel 13a): $\Psi=1, \operatorname{Re}=18.9, \Lambda=6.3$; (b) counter-current gas flow (asterisk in panel 13a): $R_{g}=-17.27, R e=14.6, \Lambda=5.7$. The gas, flowing from right to left, is constricted by the wave hump, similar to a de Laval nozzle.

mucus films in pulmonary capillaries. We see that travelling-wave solutions are lost when the magnitude of the counter-current gas flow $\left|R_{\mathrm{g}}\right|$ is increased beyond a limit point.

The effect of the gas flow on the occlusion bound $\mathrm{Re}_{\max }$ observed in panel 13a results from the pressure coupling between gas and liquid and not from the gaseous interfacial viscous stresses. This is evidenced by the curves without symbols in panel 13a, which pertain to different limits of the full-model continuation at $R_{\mathrm{g}}=-17.27$ (dashed curve). The blue solid curve without symbol pertains to the limit $\Pi_{\mu}=0$ and the dot-dashed curve to the limit $\Pi_{\rho}=0$. Deactivating the gaseous viscous stresses $\left(\Pi_{\mu}=0\right.$, solid blue curve without symbols), changes $R_{\max }$ very little. By contrast, deactivating the gas pressure effect $\left(\Pi_{\rho}=0\right.$, dot-dashed curve), almost entirely negates the gas-induced precipitation of $\mathrm{Re}_{\max }$ versus the aerostatic reference case (solid black curve with filled circle).

To elucidate the role of the gas pressure $p_{\mathrm{g}}$, panels $13 \mathrm{c}$ and $13 \mathrm{~d}$ represent wave profiles and corresponding profiles of the pressure gradient $\partial_{x} p_{\mathrm{g}}$ for the two marked limit points $(\mathrm{LP})$ in panel 13a. Thereby, $\partial_{x} p_{\mathrm{g}}$ is determined from $(2.5 c)$ and corresponds to the gas pressure gradient at the film surface $\left.\partial_{x} p_{\mathrm{g}}\right|_{r=d}$. We have dropped the accompanying subscripts for convenience. In panel $13 \mathrm{~d}, \partial_{x} p_{\mathrm{g}}$ is multiplied with $\mathrm{Fr}^{2}$, in order to normalize the actual pressure gradient with the aerostatic one. Grey/white zones between the dashed lines in panels $13 \mathrm{c}$ and $13 \mathrm{~d}$ distinguish regions of negative/positive $\partial_{x} p_{\mathrm{g}}$ in the gas. In addition, figure 14 represents streamlines in the wave-fixed reference frame corresponding to the wave profiles in panel 13c.

Focussing on the counter-current case, panel 14b, we see that the wave hump produces a constriction through which the gas (flowing from right to left) must pass, similar to a 
de Laval nozzle that first accelerates and then decelerates the flow. This produces strong positive/negative pressure gradients to the right/left of the wave maximum (see dashed line in panel 13d) and these tend to push the liquid within the film toward the wave hump (arrows in panel 13c). This mechanism tends to increase the wave amplitude for a given $R e$ and thus to advance the occlusion bound $R_{\max }$. It requires a sufficiently strong relative motion between the traveling wave and the gas. This does not necessarily require an upward gas flow. Indeed, the diamond-marked curve in panel 13b shows that occlusion may be brought about even when the gas is co-current, at least if $\left|R_{\mathrm{g}}\right|$ is sufficiently small.

In the aerostatic case, $\Psi=1$, axial pressure variations in the core are very weak (solid line in panel 13d) and the gas has no incidence on the occlusion limit. Vice versa, the flow structure in the gas is strongly affected by the liquid film. We see this in panel 14a, which pertains to case 4 (low-viscosity silicone oil), and also in panel 11a, which pertains to case 1 (high-viscosity silicone oil). In both examples, the gas flow is enslaved to the liquid surface flow (through equation $2.3 a$ ), which exhibits interfacial stagnation points around the wave hump, as a result of the moving-frame vortex in the liquid film. Due to this, the gas flow is divided into an intricate system of vortices. The constellation of these vortices is dictated by the strength of the liquid vortex in the wave hump.

\subsection{Occlusion scenarios in a spatially evolving film}

In section 3.2, we introduced the conservative occlusion bounds $R_{0}$ and $R_{\max }$ based on the limit of travelling-wave solutions and delimited three regimes for a naturally evolving wavy falling liquid film: (i) impossible occlusion, when $\operatorname{Re}<\mathrm{Re}_{0}$, (ii) conditional occlusion, when $\operatorname{Re}_{0}<\operatorname{Re}<\operatorname{Re}_{\max }$, and (iii) certain occlusion, when $\operatorname{Re}>\operatorname{Re}_{\max }$. We now demonstrate these regimes based on spatio-temporal computations with our model (2.5), where we have mimicked naturally-evolving waves through the noisy inlet condition (2.6b), using $\epsilon_{2}=10^{-5}$. For the gas, we have imposed $\Psi=1$.

We start by reproducing two of the occlusion experiments of Dao \& Balakotaiah (2000), i.e. their experimental runs 20 and 13 which identified the experimental occlusion point at $\operatorname{Re}=0.0497$ and $R e=0.258$, respectively. These two runs correspond to our cases 2 and 3 in table 1, for which we have determined the lower/upper occlusion bounds in $\S 3.2$ as $\operatorname{Re}_{0}=0.012 / \operatorname{Re}_{\max }=0.038$ (panel $5 \mathrm{c}$ ), and $\operatorname{Re}_{0}=0.075 / \operatorname{Re}_{\max }=0.39$ (panel $5 \mathrm{~d}$ ), respectively. Based on this, experimental run 20 (our case 2) lies in the region of certain occlusion and experimental run 13 (our case 3 ) in the region of conditional occlusion. This is confirmed by panels $15 \mathrm{a}$ and $15 \mathrm{~b}$, which represent travelling-wave solutions continued in terms of the wave frequency $f$ (right abscissae) and dispersion curves of the linear growth rate $\alpha_{i}$ (left abscissae) for these two runs.

The experiments of Dao \& Balakotaiah (2000) were performed in a long vertical tube equipped with a conductance probe that allowed to detect occlusion locally. However, no information on the film's surface dynamics upstream of this point was available. We provide this missing information in panels $15 \mathrm{c}$ and $15 \mathrm{~d}$, which represent snapshots of our spatio-temporal computations reproducing experimental runs 20 and 13. These computations were performed on an $L^{\star}=1 \mathrm{~m}$ domain. We see that the two cases reach occlusion by two very different routes, as a result of distinct wave dynamics.

For experimental run 20 (our case 2), most of the dispersion curve in panel 15a (grey area) lies beyond the limit point of travelling wave solutions $\left(f_{\max }<f_{\mathrm{LP}}\right)$. Thus, the most amplified surface waves emerging from the linear regime don't possess any saturated travelling state and inevitably cause occlusion by extending their growth into the nonlinear regime (panel 15c). This is occlusion scenario I, which we had already observed for case 1 (high-viscosity silicone oil) in figure 4. Supplementary movie M2 shows 
(a)

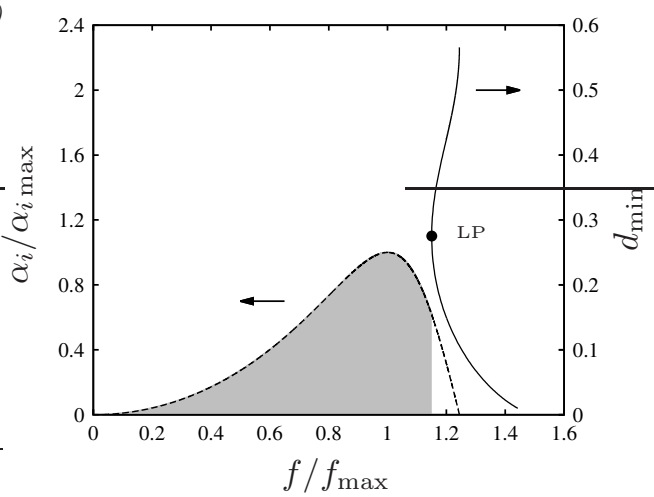

(b)

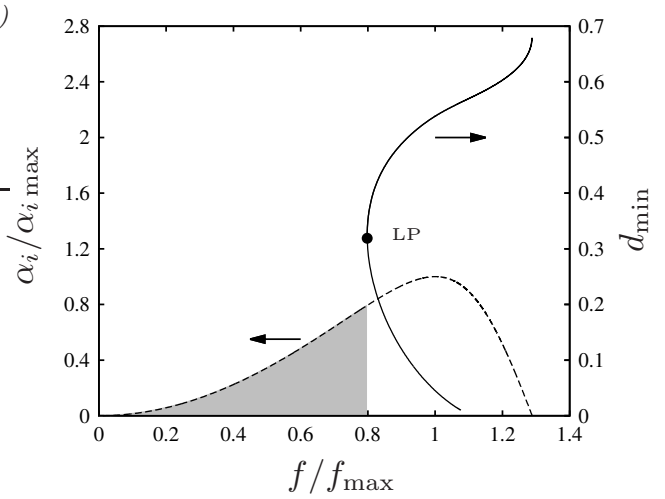

(c)

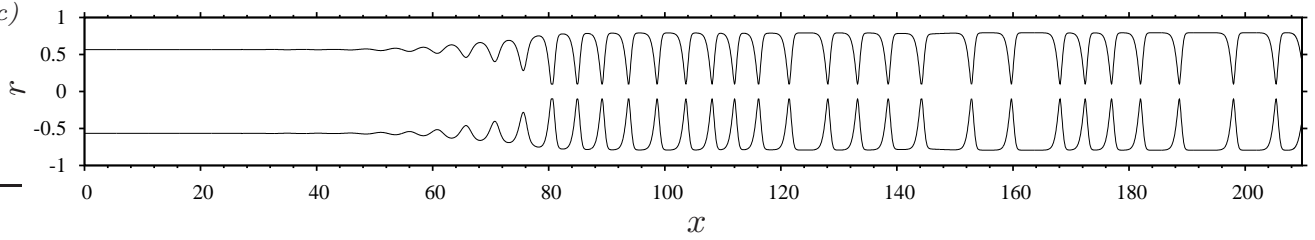

(d)

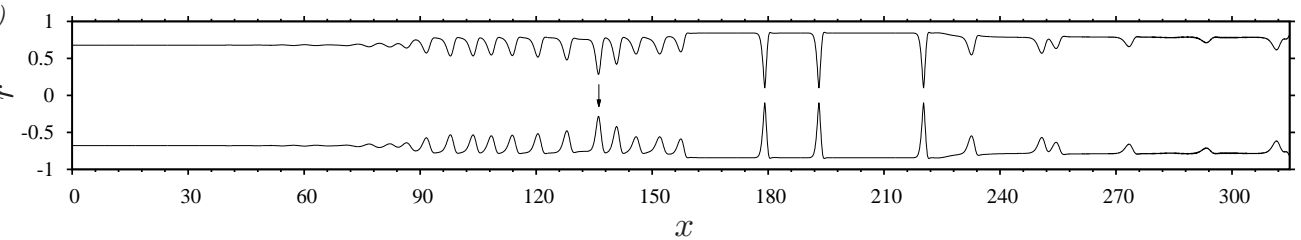

FIGURE 15. Different routes to occlusion in a spatially evolving film. Computations of experimental runs 20 (panels $a, c$ ) and 13 (panels $b, d$ ) in Dao \& Balakotaiah (2000). (a,b) Travelling wave solutions (right abscissae) at $\mathrm{Re}=$ const, and dispersion curves of the linear spatial growth rate $\alpha_{i}$ (left abscissae). Grey zones distinguish linear waves that have no saturated travelling state; $(c, d)$ spatio-temporal computations on an $L^{\star}=1 \mathrm{~m}$ domain with inlet noise $(2.6 b)$; $(a, c)$ experimental run 20: case 2 in table $1, R e=0.0497$ (regime of certain occlusion, scenario I); (b,d) experimental run 13: case 3 in table $1, R e=0.258$ (regime of conditional occlusion, scenario II). Arrow in panel $d$ marks a coalescence event triggering occlusion.

the computation from panel $15 \mathrm{c}$ in action. We remind the reader that the unphysical plug reopening observed therein (and in supplementary movie M3) is due to the crude numerical core radius limitation introduced in section 2.1. First results in appendix E suggest that this artefact is negated by our improved WRIBL model (figure 22).

By contrast, for experimental run 13 (our case 3), the most amplified linear waves lie well within the range of travelling-wave solutions $\left(f_{\max }>f_{\mathrm{LP}}\right.$ in panel 15b). Occlusion must thus occur through a different route, as evidenced by the wave profile in panel 15 d. In the first part of the tube $(x \leqslant 130)$, a quite regular train of saturated-amplitude travelling waves develops. These result from linear wave selection and their minimal core radius $d_{\min }$ is far from reaching the tube axis. However, individual waves within the wave train are quite narrowly spaced and thus prone to the secondary subharmonic/sideband instabilities typically associated with single-humped waves in planar falling liquid films (Liu \& Gollub 1993; Chang et al. 1993). These instabilities result from wave interactions triggered by residual noise and lead to wave coalescence, which is the first step in the so-called wave coarsening dynamics identified by Chang et al. (1996b). This cascaded dynamics produces ever longer, more dangerous, waves by successive coalescence/absorption events and thus represents an inherent route toward occlusion. The arrow in panel 15d 

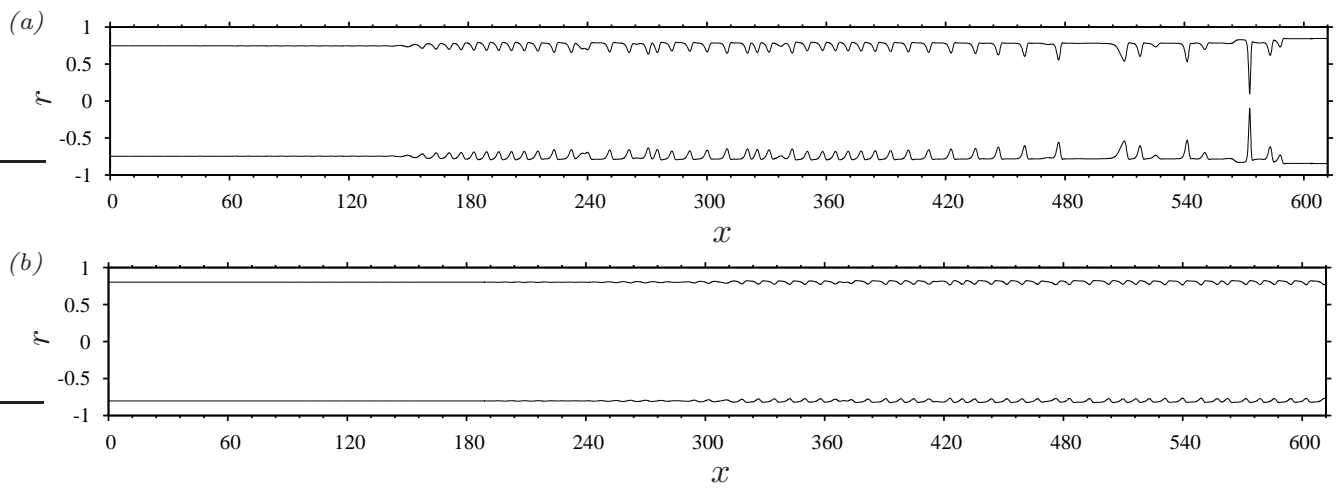

Figure 16. Computations of case $3\left(\operatorname{Re}_{0}=0.075, \operatorname{Re}_{\max }=0.39\right.$; panel $\left.5 \mathrm{~b}\right)$ at lower $\mathrm{Re}$, showing a downstream displacement of the occlusion point w.r.t. panel 15d. Spatio-temporal computations on an $L^{\star}=2 \mathrm{~m}$ domain with inlet noise $(2.6 b)$. (a) $\mathrm{Re}=0.14$ : regime of conditional occlusion. Occlusion (through scenario II) occurs much further downstream than in panel 15d; (b) $\operatorname{Re}=0.07<\operatorname{Re}_{0}$ : regime of impossible occlusion. Surface waves are extremely small.

highlights one such coalescence event, which produces a wave of greater amplitude that will occlude the tube and subsequently absorb several smaller waves downstream.

This is occlusion scenario II, which is intermittent because the initiating wave coalescence is occasional, in contrast to scenario I (panel 15c), where (almost) every wave grows to occlude the tube. Due to this intermittence, gas bubbles resulting from successive occlusion events are much more unevenly distributed. Supplementary movie M3 shows the computation from panel $15 \mathrm{~d}$ in action.

We now focus on case 3 and compute the spatio-temporal wave evolution for values of Re below the experimental occlusion threshold $R e=0.258$ determined in run 13 of Dao \& Balakotaiah (2000). This experimental threshold lies well within the region of conditional occlusion, which is bounded by $\operatorname{Re}_{0}=0.075$ and $\operatorname{Re}_{\max }=0.393$ (panel 5d).

Panel 16a represents a spatio-temporal computation at a lower Reynolds number $R e=0.14>R_{0}$, which still lies in the conditional occlusion regime. This computation was performed on a domain of $L^{\star}=2 \mathrm{~m}$ in length, i.e. twice longer than the one in panel 15d. We see that occlusion still occurs, through scenario II, but that the occlusion point has shifted greatly downstream w.r.t. panel $15 \mathrm{~d}$. The reason for this is that the frequency/wavelength of the limiting travelling waves greatly decreases/increases with decreasing Re (see panel 5d) and, thus, much more space is needed for these to develop from the coarsening dynamics. The downstream shift in the occlusion point explains why Dao \& Balakotaiah (2000), who checked for occlusion in a fixed region, found an experimental threshold for Re that is greater than the lower conservative bound $\mathrm{Re}_{0}$. It also clearly shows that occlusion in the conditional regime (through scenario II) depends on whether the tube is sufficiently long to accommodate formation of those waves that are able to cause occlusion. As these waves may be much longer than those emerging from linear selection, occlusion may require unrealistically long tube lengths, depending on Re. Supplementary movie M4 shows the computation from panel 16a in action.

Conversely, occlusion in an infinitely long tube will eventually always occur if $\mathrm{Re}$ lies within the conditional regime, at least for a naturally evolving film. This is due to the nature of the coarsening dynamics, which halts only after truly solitary waves have formed. Thus, to avoid occlusion in an infinitely long tube, Re must be decreased below the lower conservative bound $\mathrm{Re}_{0}$, where even solitary waves are too small to cause 

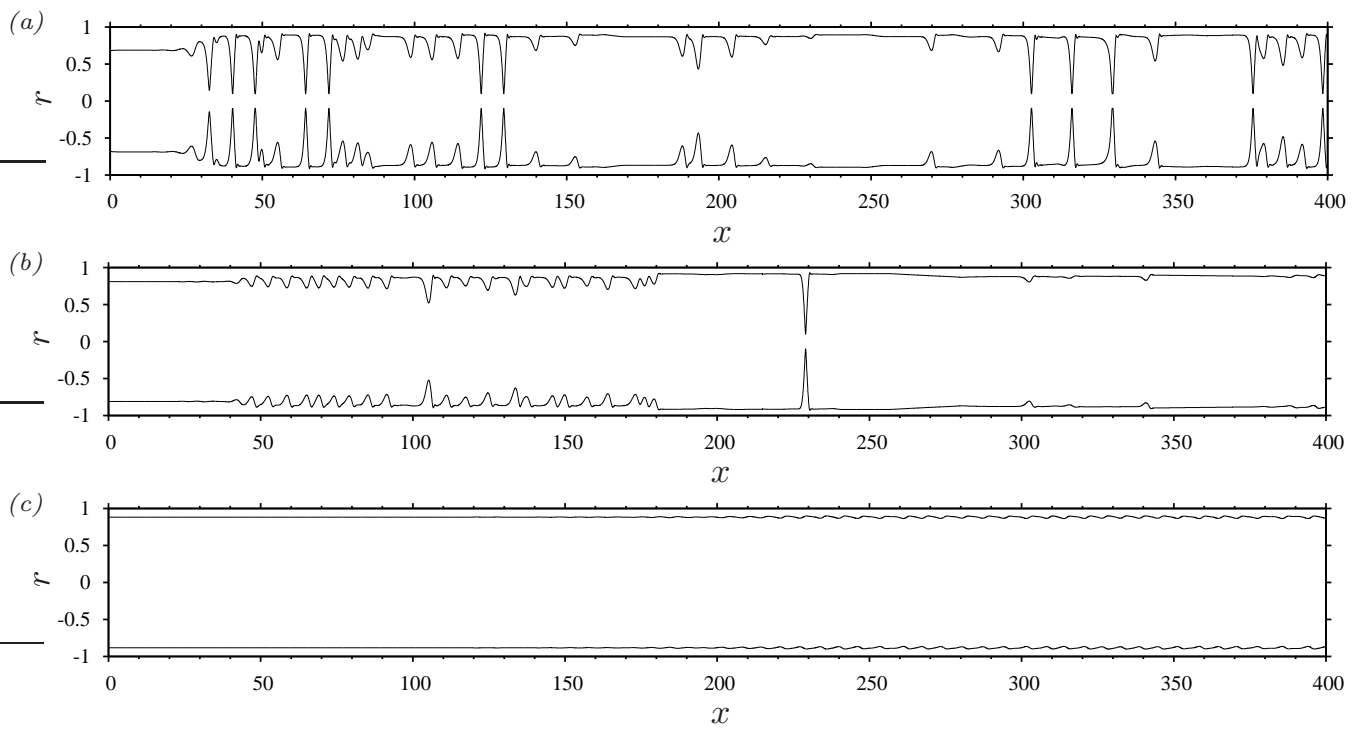

Figure 17. Different occlusion scenarios for case 4 ( $\operatorname{Re}_{0}=1.5, \operatorname{Re}_{\max }=18.9$; panel $\left.6 \mathrm{~b}\right)$. Spatio-temporal computations on an $L^{\star}=1 \mathrm{~m}$ domain with inlet noise (2.6b). (a) $R e=19>\operatorname{Re}_{\max }$ : regime of certain occlusion (scenario I); (b) $R e=5$ : regime of conditional occlusion (scenario II); (c) $\mathrm{Re}=1.25<\mathrm{Re}_{0}$ : regime of impossible occlusion. Surface waves are extremely small

occlusion. Panel $16 \mathrm{~b}$ shows a computation under such conditions, i.e. $\operatorname{Re}=0.07<\operatorname{Re}_{0}$. Although occlusion is indeed avoided, surface waves are extremely small in amplitude, which thwarts their beneficial effect on heat and mass transfer (Yoshimura et al. 1996; Albert et al. 2013). This underlines the conservative nature of the lower occlusion bound $\mathrm{Re}_{0}$. We will show in the next section that there is another way to prevent occlusion, namely through coherent inlet forcing in the conditional occlusion regime, which allows maintaining surface waves of significant amplitude.

Finally, figure 17 shows snapshots of three representative spatio-temporal computations for case 4 (low-viscosity silicone oil), where inertia, which drives the the Kapitza instability, is relevant. Panels 17a, 17b, and 17c correspond to the regimes of certain, conditional, and impossible occlusion. These display the same main features observed for the high-viscosity liquids (panels 15c, 16a, and 16b), only that the length scales of the occlusion processes are much shorter.

\subsection{Coherent inlet forcing to prevent occlusion}

For $\mathrm{Re}>\mathrm{Re}_{0}$, travelling-wave solutions are bounded by a limiting frequency $f_{\mathrm{LP}}$, below which they cannot exist and occlusion is inevitable (see panels 5d and 6b). Conversely, by increasing $f$ above $f_{\mathrm{LP}}$, travelling-wave solutions are recovered and occlusion can be avoided. We thus test the idea of forcing high-frequency waves through coherent inlet forcing in a spatially-evolving falling liquid film, in order to prevent occlusion without having to reduce Re below the conservative bound $\mathrm{Re}_{0}$, where surface waves all but disappear (see panels $16 \mathrm{~b}$ and $17 \mathrm{c}$ ).

This idea works only in the conditional occlusion regime, $\operatorname{Re}_{0}<\operatorname{Re}<\operatorname{Re}_{\max }$. Indeed, in the regime of certain occlusion, $\operatorname{Re}>\operatorname{Re}_{\max }$, where $f_{\mathrm{LP}}>f_{\max }$, one would have to force waves of frequency $f>f_{\max }$. Such short waves are very sensitive to the secondary instabilities causing wave coalescence in closely-packed wave trains (Liu \& Gollub 1993), 

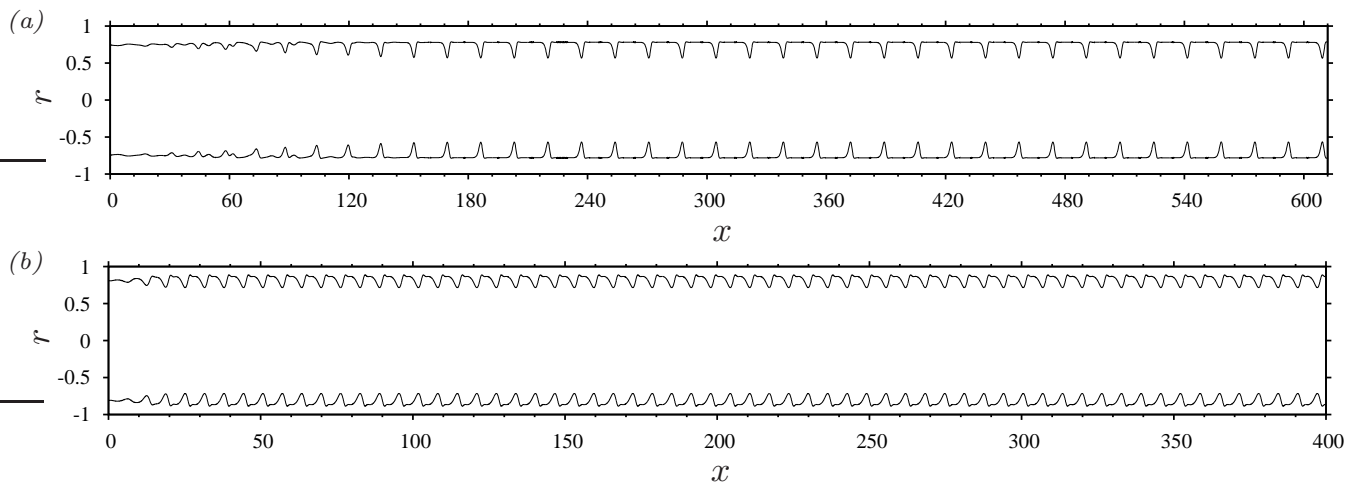

FiguRE 18. Preventing occlusion in the conditional occlusion regime, $\operatorname{Re}_{0}<\operatorname{Re}<\operatorname{Re}_{\max }$, through coherent inlet forcing. Spatio-temporal simulations from panels $16 \mathrm{a}$ and $17 \mathrm{~b}$ with additional coherent inlet forcing of frequency $f$ and amplitude $\epsilon_{1}=0.1(2.6 b)$. All other parameters, including the noise level $\epsilon_{2}$, remain unchanged. (a) Case $3\left(\operatorname{Re}_{0}=0.075, \mathrm{Re}_{\max }=0.393\right.$; panel $\left.5 \mathrm{~d}\right): \mathrm{Re}=0.14$, $f=0.5 f_{\max }=0.525 ;$ (b) Case $4\left(\operatorname{Re}_{0}=1.5, R_{\max }=18.9\right.$; panel $\left.6 \mathrm{~b}\right): \operatorname{Re}=5, f=0.5 f_{\max }=1.27$ Coherent inlet forcing produces a regular train of saturated-amplitude travelling waves, allowing to prevent occlusion versus the natural evolution in panels $16 \mathrm{a}$ and $17 \mathrm{~b}$.

and rapidly loose the signature of the inlet forcing. By contrast, in the conditional occlusion regime, $f_{\mathrm{LP}}$ can be low enough (the lower Re, the lower $f_{\mathrm{LP}}$ ) to allow forcing a wave train that is not subject to secondary instability.

We demonstrate this by running the spatio-temporal computations in panels 16a (case 3 ) and $17 \mathrm{~b}$ (case 4) again with additional coherent inlet forcing. For this, we activate the monochromatic perturbation in $(2.6 b)$ with a relative amplitude $\epsilon_{1}=0.1$. Meanwhile, the inlet noise remains active and its level unchanged $\epsilon_{2}=10^{-5}$. We stress that $\epsilon_{2}$ in $(2.6 b)$ is the scale factor of a Fourier series which 1000 terms. Thus, the actual noise amplitude is much greater than suggested by the value of $\epsilon_{2}$. It is about $10 \%$ of $\epsilon_{1}$ for the runs presented here. The forcing frequency $f$ is chosen greater than the limit value $f_{\mathrm{LP}}$ of travelling-wave solutions, as obtained from panels $5 \mathrm{~d}$ (case $3, \mathrm{Re}=0.14, f_{\mathrm{LP}}=0.41 f_{\max }$ ) and $6 \mathrm{~b}$ ( case $4, \mathrm{Re}=5, f_{\mathrm{LP}}=0.35 f_{\max }$ ), respectively.

Panels $18 \mathrm{a}$ and $18 \mathrm{~b}$ show the results of our spatio-temporal computations with additional coherent inlet forcing and are to be compared directly to panels $16 \mathrm{a}$ and $17 \mathrm{~b}$, where the wave dynamics is purely noise-driven. We see that the additional coherent inlet forcing produces a regular train of saturated-amplitude travelling waves that do not occlude the tube. This wave train is maintained over the entire length of the domain and thus robust w.r.t. to secondary instability. In contrast to panels $16 \mathrm{~b}$ and $17 \mathrm{c}$, where occlusion was avoided by reducing Re below the lower conservative bound $\mathrm{Re}_{0}$, the falling films in panels $18 \mathrm{a}$ and $18 \mathrm{~b}$ display quite substantial surface waves, expected to be much more beneficial for inter-phase heat and mass transfer.

\section{Conclusion}

We have studied numerically the occlusion of a narrow vertical cylindrical tube by an axisymmetric wavy falling liquid film in contact with a laminar core gas flow. We have focused on conditions where the effect of gravity is at least comparable to that of capillarity, i.e. $B o \geqslant 1$, and the gas Reynolds number $\operatorname{Re}_{\mathrm{g}}$ is quite low. In that limit, occlusion is caused by spatially growing waves and not by absolute linear instability. 
These waves are generated by a combination of the Plateau-Rayleigh instability, gravitydriven advection, and the Kapitza instability.

Using the low-dimensional model introduced in Dietze \& Ruyer-Quil (2015), which had been applied only to gravity-free films, we have performed spatio-temporal computations of spatially-evolving falling films and computed travelling-wave solutions through numerical continuation. Our model extends upon the earlier works of Trifonov (1992), Camassa et al. (2014, 2017), Zhou et al. (2016), Liu \& Ding (2017), and Ding et al. (2019) by accounting for axial viscous diffusion and inertia. These effects are needed to accurately predict occlusion for the real liquids studied here (cases 1-4 in table 1). We have validated our model computations with DNS (appendix A and panel 5d).

Travelling-wave solutions are lost when the liquid Reynolds number Re is increased beyond a limit point (LP), low-frequency/long waves being more dangerous (smaller $\operatorname{Re}_{L P}$ ) than high-frequency/short waves (greater $\mathrm{Re}_{\mathrm{LP}}$ ). By numerically reproducing the occlusion experiments of Camassa et al. (2014), we have proved that surface waves causing occlusion systematically lie beyond this limit of travelling-wave solutions, thus validating the conjecture formulated by these authors.

Depending on how occluding waves emerge in a spatially evolving film, either directly from linear wave selection (scenario I), or subsequent secondary instability and wave coarsening (scenario II), we have distinguished two possible occlusion scenarios. By reproducing several occlusion experiments with our own spatio-temporal computations, we have shown that occlusion scenario I (panels $4 \mathrm{a}$ and $15 \mathrm{c}$, movie M2) applies to the experiments of Camassa et al. (2014) and run 20 in Dao \& Balakotaiah (2000), while scenario II (panel 15d, movie M3) applies to run 13 in Dao \& Balakotaiah (2000).

We have delimited scenarios I and II based on an upper conservative occlusion bound $\mathrm{Re}_{\max }$, which corresponds to the limit point of travelling-wave solutions at the spatially most amplified frequency $f_{\max }$ of linear waves. And, a lower conservative bound $\mathrm{Re}_{0}$, below which travelling-wave solutions always exist, no matter how great the wavelength (Ding et al. 2019). Determining $\mathrm{Re}_{\max }$ requires computing $f_{\max }$ and thus we have simultaneously solved the linear stability problem in our numerical continuations. This had not been attempted in previous studies (Camassa et al. 2014; Ding et al. 2019).

Based on the upper and lower conservative bounds $\mathrm{Re}_{\max }$ and $\mathrm{Re}_{0}$, as well as spatiotemporal computations, we have delimited three possible regimes for a naturally evolving wavy film: (i) certain occlusion: $R e>R_{\max }$. The most amplified surface waves, which typically emerge in an experiment, do not possess travelling states. Occlusion is inevitable and occurs through scenario I; (ii) conditional occlusion: $\operatorname{Re}_{0}<\operatorname{Re}<\operatorname{Re}_{\max }$. Occlusion is theoretically possible through scenario II (for long enough waves), but does not necessarily occur in a real system. That depends on whether the coarsening dynamics can produce sufficiently long waves and whether the tube is long enough to accommodate this (panels 15d and 16a, movies M3 and M4); (iii) impossible occlusion: $\operatorname{Re}<\operatorname{Re}_{0}$. All possible surface waves, no matter how long, are safe.

We have shown that occlusion can be actively prevented in the regime of conditional occlusion $\left(\operatorname{Re}_{0}<\operatorname{Re}<\operatorname{Re}_{\max }\right)$ by forcing waves of sufficiently high frequency through coherent inlet forcing (panels 18a and 18b). This allows to maintain surface waves of nonnegligible amplitude, in contrast to the regime of impossible occlusion $\left(\operatorname{Re}<\mathrm{Re}_{0}\right)$ where waves are almost invisible (panels $16 \mathrm{~b}$ and $17 \mathrm{c}$ ). Given the well documented positive effect of surface waves on inter-phase heat/mass transfer (Yoshimura et al. 1996), coherent inlet forcing is an attractive route to enhance transfer while avoiding occlusion.

We have computed the lower and upper conservative bounds $\mathrm{Re}_{0}$ and $\mathrm{Re}_{\max }$ for the four working liquids considered here (figures 5 and 6 ). Further, we have quantified how key parameters (Bo and $R_{\mathrm{g}}$ ) as well as several physical effects (axial viscous diffusion 
and inertia) affect the upper conservative bound $\mathrm{Re}_{\max }$, which delimits the regimes of certain and conditional occlusion. We have found that gravity greatly delays this bound, significantly increasing $\mathrm{Re}_{\max }$ as the Bond number Bo is increased (see panel 8a). Unless Bo is very large, the height of travelling waves at $R e=R e_{\max }$ is far from reaching the tube radius. In contrast to what Dao \& Balakotaiah (2000) conjectured, such solutions are thus lost abruptly and not due to the wave height reaching the tube radius continuously.

We have found that a laminar counter-current gas flow can significantly precipitate occlusion for low-viscosity liquids (low-viscosity silicone oil, case 4), where the effect of the gas pressure becomes relevant in the liquid force balance. At the strongest gas flow studied $\left(R_{\mathrm{g}}=-17\right)$, we have observed a $25 \%$ reduction of $\mathrm{Re}_{\max }$ (panel 13a) versus a situation where the gas is quiescent. We have found that the gas affects the liquid film mainly through inter-phase pressure coupling. Surface waves locally constrict the gas flow, which is accelerated and then decelerated as it passes through (panel 14b). This produces a pressure minimum above the wave crest that sucks liquid toward the wave, tending to increase its amplitude and, thus, the danger of occlusion.

For high-viscosity liquids (high-viscosity silicone oil and aqueous glycerol solution, cases 1 and 3), axial viscous diffusion greatly precipitates occlusion, i.e. greatly reduces $\mathrm{Re}_{\max }$ (by $74 \%$ in panel 9a). This may be relevant for mucus films within the first five generations of the human respiratory network, where the capillary radius is large enough for gravity to be relevant (King \& Macklem 1977; Lewis et al. 2005; Grotberg 2011).

For low-viscosity liquids (low-viscosity silicone oil, case 4), we have observed that $\mathrm{Re}_{\max }$ becomes large enough for inertia to be relevant $\left(\mathrm{Re}_{\max }=18.9\right.$ in panel 6a). This activates the Kapitza instability, which tends to increase the amplitude of travellingwaves, increasing the danger of occlusion. We have found that inertia significantly precipitates occlusion, decreasing $\mathrm{Re}_{\max }$ versus an inertialess computation (by $20 \%$ in panel 12a). This may be relevant for falling film micro-reactors (Seebauer et al. 2012).

Finally, we have found that neglecting axial viscous diffusion (high-viscosity liquids, panel 9a) or inertia (low-viscosity liquids, panel 12a) can change the nature of the occlusion mechanism, from being dictated by the loss of travelling-wave solutions to being dictated by absolute linear instability.

Our transient computations on long open domains have been performed with a crude numerical core radius limitation allowing to continue these past occlusion events. Although this technique does not affect the wave dynamics leading up to occlusion, which is the focus of our current study, it does not represent the physics of liquid plugs to satisfaction. To remedy this, we have introduced an improved version of our WRIBL model (2.5) by augmenting it with an additional force term (4.1), allowing to form stable travelling pseudo-plugs (figure 22). First results reported in appendix E suggest that this improved model negates the main artefact of the numerical core radius limitation, i.e. the reopening of liquid plugs. Confronting our improved model with full-fledged plug models (Ubal et al. 2008; Suresh \& Grotberg 2005) is an enticing prospect for future work.

\section{Appendix A: Validation of WRIBL model based on transient DNS}

Figure 19 represents comparisons between our WRIBL model (2.5) and DNS using the solver Gerris (Popinet 2009). All computations were performed for case 4 (low-viscosity silicone oil), which is the most challenging. The solid and dashed curves in panel 19a represent travelling-wave solutions obtained with our model by continuing the liquid volume $V_{1}$ at fixed wavelength $\Lambda=5.4$ for two different gas flow situations. The dashed line corresponds to an aerostatic gas pressure drop $\Psi=1$, and the solid line to a counter-current gas flow at $\operatorname{Re}_{\mathrm{g}}=-17.3$. We compare these data with two punctual DNS at $V_{1} / \pi / R^{3}=2.35$, 
(a)

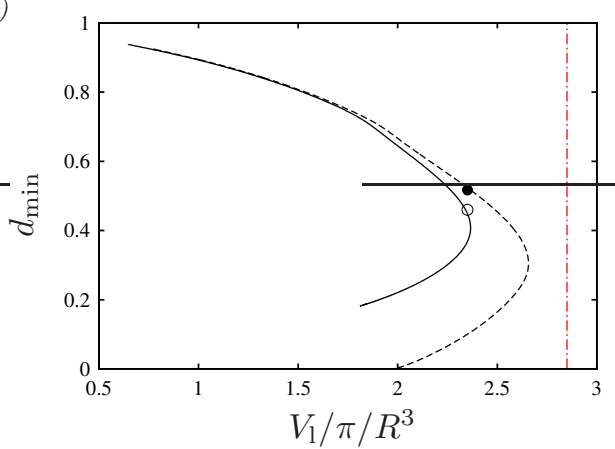

(c)

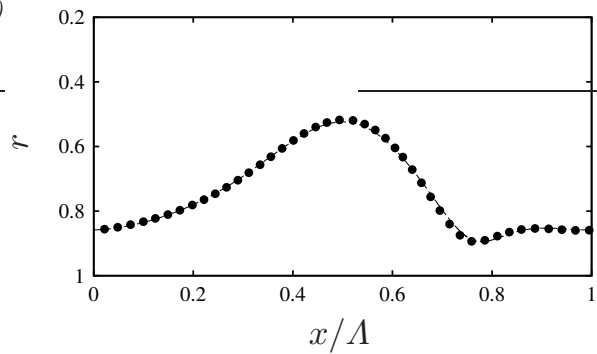

(b)

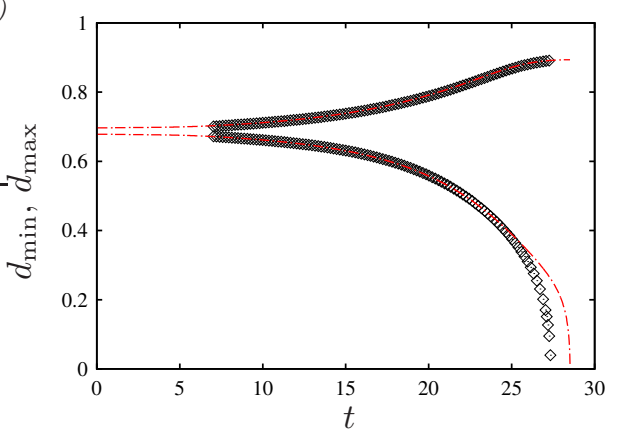

(d)

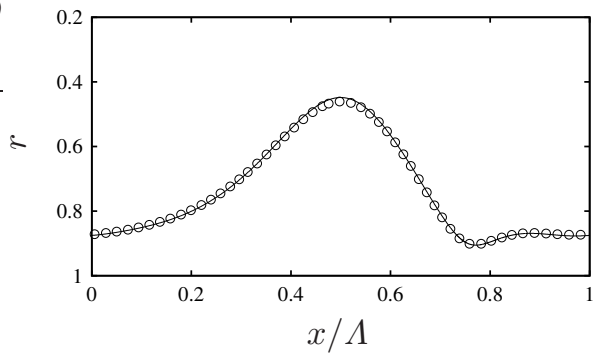

(e)

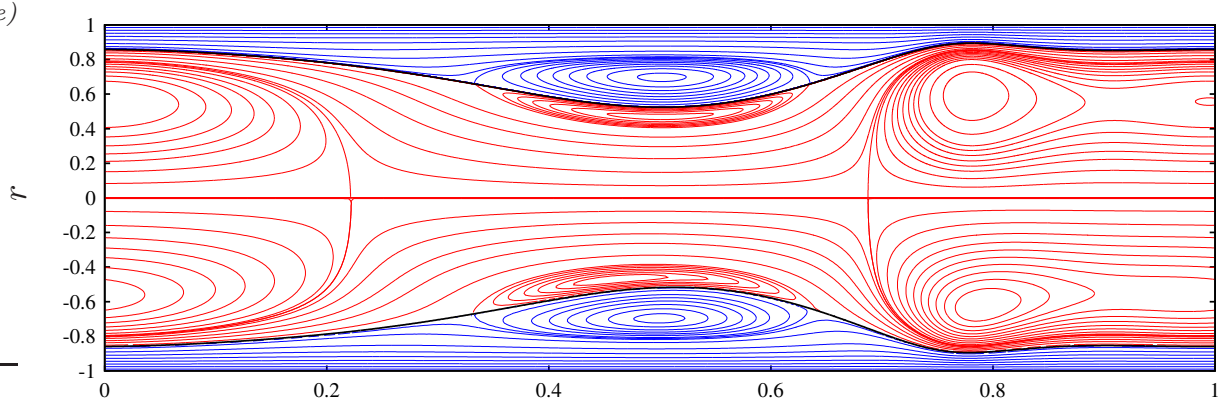

(f)

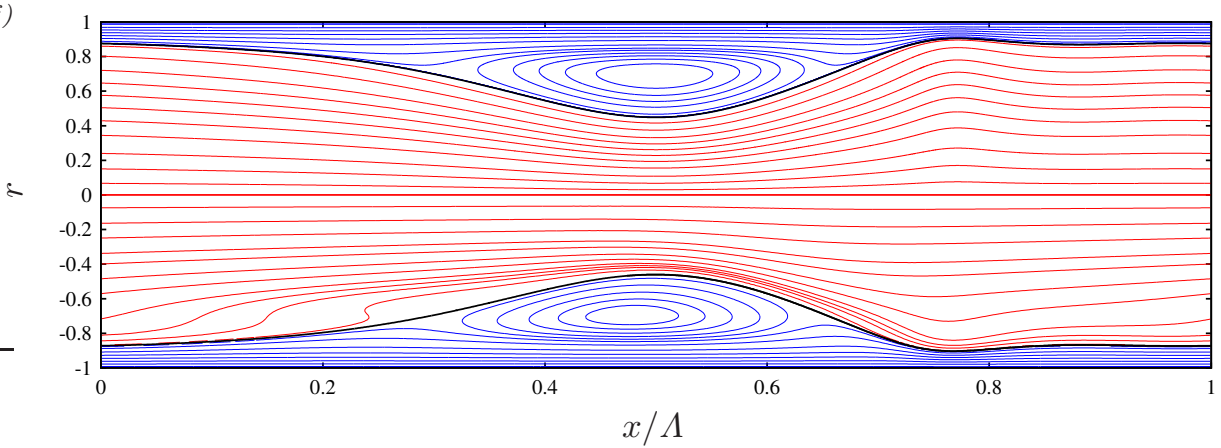

Figure 19. Validation of our WRIBL model (2.5) with DNS using the solver Gerris (Popinet 2009). Case 4 in table 1: low-viscosity silicone oil, $R^{\star}=1.5 \mathrm{~mm}$. (a) Travelling-wave solutions at fixed $\Lambda=5.4$. Dashed line (WRIBL) and filled circle (DNS, $V_{1} / \pi / R^{3}=2.35$ ): aerostatic pressure drop, $\Psi=1$; solid line (WRIBL) and open circle (DNS, $V_{1} / \pi / R^{3}=2.35$ ): $\operatorname{Re}_{\mathrm{g}}=-17.3$; (b) transient periodic computations with DNS solver (diamonds) and WRIBL model (dot-dashed line): $\Lambda=5.4$, $\Psi=1, V_{1} / \pi / R^{3}=2.85$ (vertical line in panel $a$ ); $(c-d)$ profiles of travelling waves from panel $a$ for $V_{1} / \pi / R^{3}=2.35$. Filled/open circles: DNS; dashed/solid lines: WRIBL; $(e-f)$ streamlines in the wave-fixed reference frame for solutions in panels $c$ and $d$. Top half: WRIBL; bottom half: DNS. 
which are marked by a filled $(\Psi=1)$ and open circle $\left(\operatorname{Re}_{\mathrm{g}}=-17.3\right)$, respectively. Panels $19 \mathrm{c}$ and $19 \mathrm{~d}$ represent the corresponding surface profiles at this point, as obtained from DNS (symbols) and our model (lines). Panels 19e and 19f represent corresponding streamline patterns in the wave-fixed reference frame. The top half of these graphs represents our model computations and the bottom half our DNS.

Finally, panel $19 \mathrm{~b}$ compares time traces of the minimal and maximal core radius $d_{\text {min }}$ and $d_{\max }$ obtained from transient periodic computations with our model (dot-dashed line) and the DNS solver (diamonds) for $\Lambda=5.4, \Psi=1$, and $V_{l} / \pi / R^{3}=2.85$ (vertical line in panel 19a), which lies beyond the limit point of travelling-wave solutions in panel 19a. Agreement between the two data sets in panel 19b is good and both computations produce an occlusion of the tube by the liquid film, as evidenced by the divergence of the minimal core radius $d_{\text {min }}$. This confirms that occlusion occurs beyond the limit of travelling-wave solutions and that our model is able to capture the associated dynamics.

\section{Appendix B: Numerical procedure for travelling-wave DNS}

The DNS results in panel $5 \mathrm{~d}$ were obtained by solving the full governing equations (2.1)-(2.3) in the limit $\Pi_{\mu}=\Pi_{\rho}=0$ with a pseudo-spectral approach. For this, the liquid velocity and pressure fields are projected on modified Chebyshev polynomials:

$$
u_{\mathrm{l}}(x, r, t)=\sum_{i=1}^{n} a_{i}(x, t) \phi_{i}(X), \quad p_{\mathrm{l}}(x, r, t)=\sum_{i=0}^{n-1} b_{i}(x, t) \psi_{i}(X),
$$

where $X=2(R-r) /(R-d)-1$. The radial velocity $v_{\mathrm{l}}$ is obtained by integrating $(2.1 b)$, i.e. $v_{\mathrm{l}}=-\frac{1}{r} \int_{R}^{r} \partial_{x} u_{\mathrm{l}} r d r$. The functions $\phi_{i}(X)$ and $\psi_{i}(X)$ are linear combinations of Chebyshev polynomials of the first kind $T_{i}$ :

$$
\begin{gathered}
\phi_{1}=1+X, \psi_{0}=1, \psi_{1}=X-1, \\
\phi_{2 i}=\psi_{2 i}=T_{2 i}(X)-1, \phi_{2 i+1}=\psi_{2 i+1}=T_{2 i+1}(X)-X \quad \forall i \geqslant 1,
\end{gathered}
$$

so that $\phi_{i}(X=-1)=0$, and thus the boundary conditions (2.2) are fulfilled.

Writing the Navier-Stokes equations $(2.1 a)$ on the Gauss-Lobato points $X_{i}=-\cos (\pi i / n)$ for $i \geqslant 1$, and introducing the wave-fixed coordinate $\xi=x-c t$, yields $2(n-1)$ relations:

$$
\begin{gathered}
\sum_{j=0}^{n-1} \psi_{j}\left(X_{i}\right) \mathrm{D}_{\xi} b_{j}=\mathcal{F}_{i}\left(b_{i}, a_{i}, \mathrm{D}_{\xi} a_{i}, \mathrm{D}_{\xi \xi} a_{i}, h, \mathrm{D}_{\xi} h, \mathrm{D}_{\xi \xi} h\right), \\
\frac{1}{4 \operatorname{Re}} \frac{h}{r} \sum_{j=1}^{n}\left[\mathcal{C}_{j}\left(X_{i}\right)+I_{j}\left(X_{i}\right)\right] \mathrm{D}_{\xi \xi \xi} a_{i}=\mathcal{G}_{i}\left(b_{i}, a_{i}, \mathrm{D}_{\xi} a_{i}, \mathrm{D}_{\xi \xi} a_{i}, h, \mathrm{D}_{\xi} h, \mathrm{D}_{\xi \xi} h\right),
\end{gathered}
$$

where $\mathrm{D}_{\xi}=\mathrm{d} / \mathrm{d} \xi, \mathcal{C}_{j}=\int_{-1}^{X} X\left(\phi_{j}\right)^{\prime} d X$, and $I_{j}=\int_{-1}^{X} \phi_{j} d X$. Further, we have:

$$
\begin{gathered}
\tau_{1}^{\mathrm{t}}=\eta \mathrm{D}_{\xi \xi \xi} a_{1}, \\
\tau_{1}^{\mathrm{n}}+\operatorname{Re} p_{\mathrm{l}}-\operatorname{ReWe}^{-1} \kappa=\eta \mathrm{D}_{\xi} b_{0}, \\
q_{\mathrm{l}}+c \pi d^{2}=\eta \mathrm{D}_{\xi \xi \xi} h,
\end{gathered}
$$

and thus the free-surface dynamic conditions $(2.3 b)-(2.3 c)$ and the integral continuity equation $(2.5 a)$ are recovered in the limit $\eta \rightarrow 0$. We finally obtain the linear equation system:

$$
\mathbf{A} \frac{\mathrm{d} \mathbf{U}}{\mathrm{d} \xi}=\mathbf{B}(\mathbf{U} ; \eta)
$$


(a)

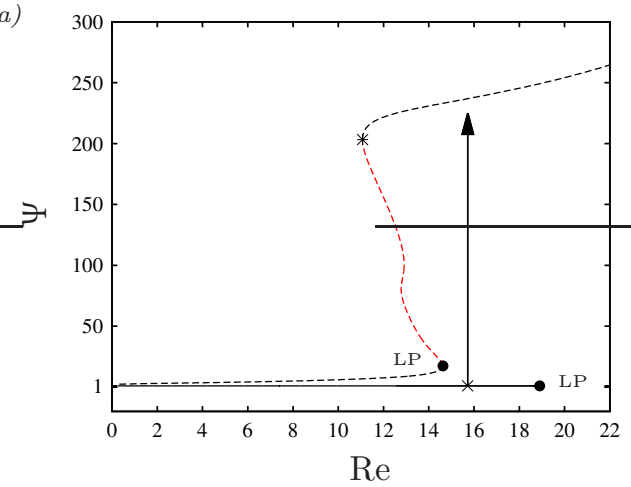

(b)

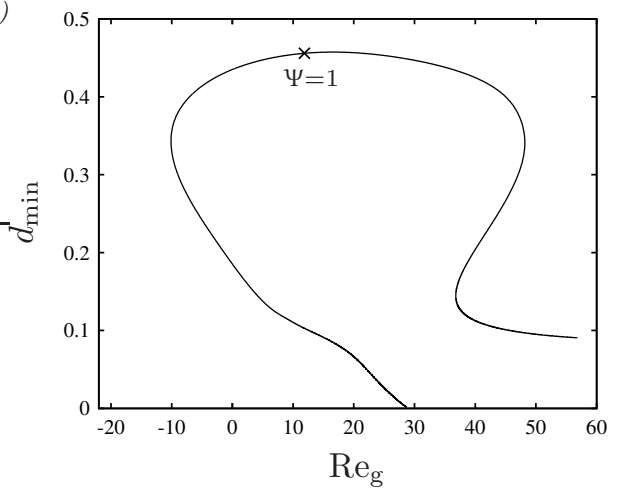

Figure 20. Travelling waves under the effect of a counter-current gas flow: case $4, f=f_{\max }$. (a) New representation of the curves from panel 13a in terms of the normalized pressure gradient $\Psi=\Delta p_{\mathrm{g}} \mathrm{Fr}^{2}$. Solid: aerostatic pressure drop, $\Psi=1$; dashed: counter-current gas flow, $\mathrm{Re}_{\mathrm{g}}=-17.27$. Solutions between circle and asterisk (red segment) are stable. Filled circles correspond to limit points (LP) in panel 13a; (b) variation of the gas Reynolds number $R_{\mathrm{g}}$ at $R \mathrm{e}=15.72$. The intended continuation path is indicated by an arrow in panel $a$.

for the unknowns $\mathbf{U}=\left(h, \mathrm{D}_{\xi} h, \mathrm{D}_{\xi \xi} h, a_{i}, \mathrm{D}_{\xi} a_{i}, \mathrm{D}_{\xi \xi} a_{i}, b_{i-1}\right) \forall 1 \leqslant i \leqslant n$. Inverting (4.5) leads to an autonomous dynamical system of dimension $4 n+3$. This dynamical system was solved with the continuation software AUTO07P (Doedel 2008), using a predictorcorrector method (Kalliadasis et al. 2012). Our computations were performed with $\eta=10^{-6}$ and $n=20$, which we have determined based on convergence tests.

\section{Appendix C: Travelling waves on the lower branch in panel 13a}

We discuss further the travelling-wave solutions on the lowest branch of the dashed curve in panel $13 \mathrm{a}$, which extends beyond the limit point $R e=R_{\max }$, as the result of interfacial viscous stresses exerted by the gas. Panel 20a replots that curve (dashed line) and the corresponding curve for the aerostatic configuration (solid line) in terms of the pressure gradient $\Psi=\Delta p_{\mathrm{g}} \mathrm{Fr}^{2}$. The filled circles therein correspond to the limit points (LP) in panel 13a. The segment of the dashed curve that we are interested in lies above the asterisk, and we have checked the stability of solutions thereon, using transient periodic computations with imposed $\Psi$ (we recall that the red portion of the curve, between circle and asterisk, is stable). As Re is increased on this curve segment, solutions go from being stable to being subject to an oscillatory instability, similar to that found in planar channels (Lavalle et al. to be published). In both situations, our transient periodic computations do not produce occlusion.

However, these states are inaccessible in a real system, where Re is fixed and the counter-current gas flow is imposed through $\Psi$. The arrow in panel 20a indicates how $\Psi$ would need to be varied to attain the desired states, starting from the aerostatic situation (marked by a cross). Besides that this would require a two hundred fold increase of $\Psi$, it turns out that there is no continuation path (at $\mathrm{Re}=$ const) from the solid curve, where $\Psi=1$, to the dashed curve, where $R_{\mathrm{g}}=-17.27$. This is shown in panel $20 \mathrm{~b}$, representing a continuation of travelling-wave solutions at $R e=15.72$, where we have varied $R_{\mathrm{g}}$. The solution curve exhibits a limit point at $\mathrm{Re}_{\mathrm{g}} \approx-10$ that precludes reaching the target value $\operatorname{Re}_{\mathrm{g}}=-17.27$.

We have also performed a transient computation on an open domain with inlet/outlet conditions, where we have set $\operatorname{Re}=15.72$ and forced waves of frequency $f=f_{\max }\left(\operatorname{Re}_{\mathrm{g}}=\right.$ 


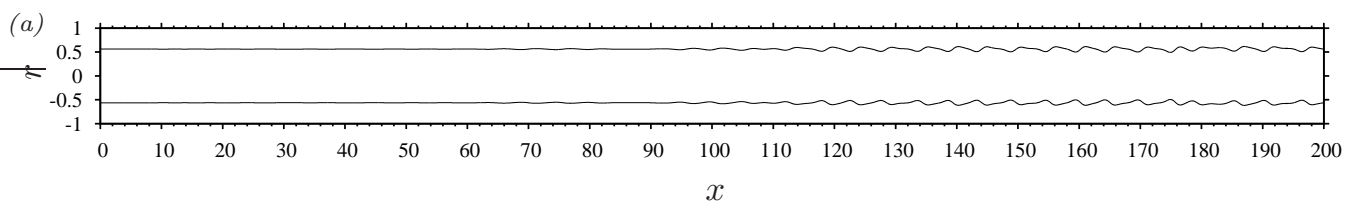

Figure 21. Version of the computation from panel 4a with axial viscous diffusion deactivated: $J_{j}=K_{j}=L_{j}=M_{j}=0$ in (2.5). The film surface is represented at the same time as in panel $4 \mathrm{a}$.

$-17.27)=0.73$, while gradually increasing $\Psi$ from $\Psi=1$ according to a sigmoid function. This computation produces occlusion long before $\Psi$ has reached the target value $\Psi=237$, which corresponds to the upper solution branch in panel 20a. We may thus conclude that $\mathrm{Re}_{\max }$, which is based on the limit point (LP) in panel 13a, remains a representative occlusion bound also in the counter-current configuration.

\section{Appendix D: Effect of axial viscous diffusion in panel 4a}

Figure 21 represents a version of the computation from panel 4a where we have deactivated axial viscous diffusion, by setting $J_{j}=K_{j}=L_{j}=M_{j}=0$ in $(2.5 b)$. In contrast to panel $4 \mathrm{a}$, no occlusion is observed over the entire length of the tube, which is in qualitative contradiction with the experiments of Camassa et al. (2014).

\section{Appendix E: Improved representation of liquid plugs}

Inspired by the use of an additional attractive-repulsive force term to model contact line problems within the framework of film models (Thiele et al. 2001), we add a repulsive azimuthal capillary term to the RHS of our model equation $(2.5 b)$ :

$$
\Pi_{\theta}=\mathrm{We}^{-1} \exp \left[\lambda\left(1-\frac{d(x, t)}{d_{\text {plug }}}\right)\right] \partial_{x} \kappa_{\theta}, \quad \kappa_{\theta}=-\frac{1}{d}\left[1-\frac{1}{2}\left(\partial_{x} d\right)^{2}\right] .
$$

As a result of its exponential variation with $d$ (which can be scaled with $\lambda$ ), the effect of this term is significant only in the vicinity of the predefined radius $d_{\text {plug }}$, which is set to a small fraction of the tube radius $R$. At $d=d_{\text {plug }}, \Pi_{\theta}$ exactly compensates the capillary pressure gradient due to (destabilizing) azimuthal curvature. Furthermore, by adequately choosing $d_{\text {plug }}$ and $\lambda$, the primary flow with a cylindrical film surface at $d=d_{\text {plug }}$ can be rendered linearly stable at all wave numbers (we have checked this with spatial linear stability analysis). This allows representing liquid plugs as stable travelling liquid annuli, or pseudo-plugs, that almost entirely fill the tube cross section.

Figure 22 represents results of a transient periodic computation based on our WRIBL model (2.5) with the additional force term $\Pi_{\theta}(4.1)$. Liquid properties and tube radius correspond to case 4 , which, due to the relevance of inertia, is the most challenging. Further, $\Lambda=5.40, V_{1} / \pi / R^{3}=2.80, d_{\text {plug }}=0.01 R$, and $\lambda=1$. Panel 22a represents the time evolution of the film surface during formation of a pseudo-plug, and panel $22 \mathrm{~b}$ represents the fully-developed pseudo-plug. To better guide the eye, we have reproduced and shifted the periodic surface profile in these panels.

In contrast to the crude numerical core radius limitation employed in our open-domain computations, our improved representation of liquid plugs ensures volume conservation, and thus the pseudo-plug in figure 22 does not reopen. On the downside, this new representation requires a finer spatial numerical resolution. Thus, open-domain computations, such as the one in panel 17a, become more costly (about four times for case 4). This can 

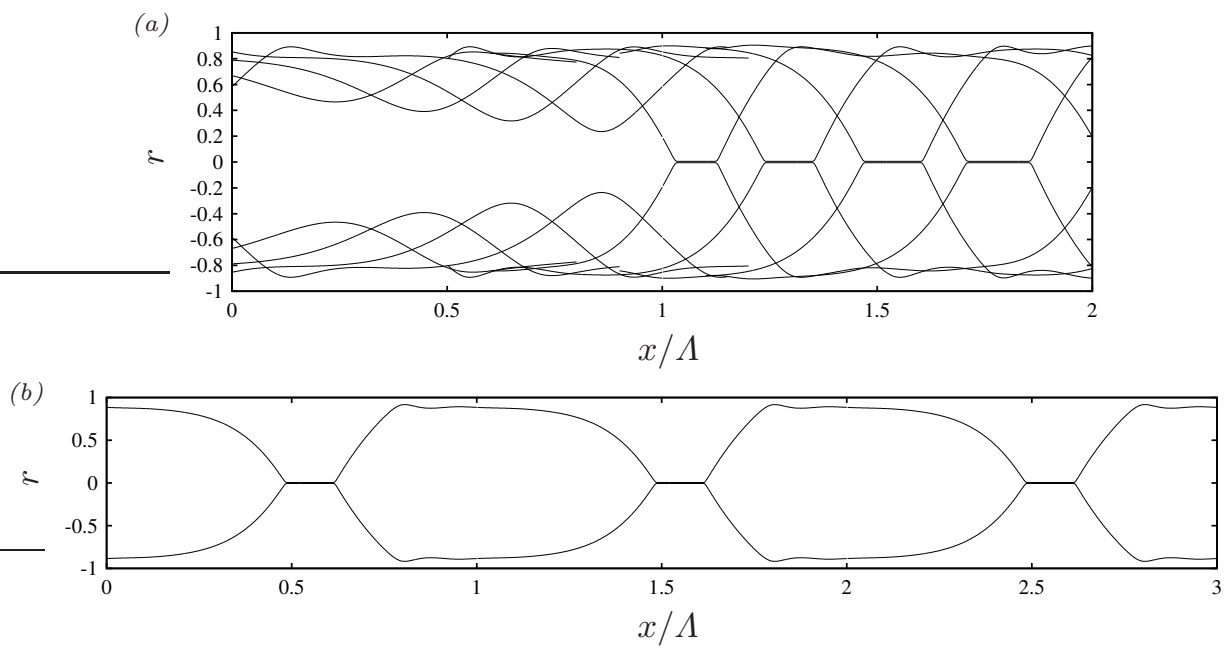

FiguRE 22. Improved representation of liquid plugs. Transient periodic computation based on the WRIBL model (2.5) upgraded with the localized repulsive term $\Pi_{\theta}(4.1)$, using $d_{\text {plug }}=0.01 R$ and $\lambda=1$. Case 4: $\Lambda=5.40 ; V_{1} / \pi / R^{3}=2.80$. (a) Surface profiles at times $t^{\star} / t_{\nu}^{\star}=20,30,40,50,60$, $70,80,90\left(t_{\nu}^{\star}=\nu_{1}^{1 / 3} g^{-2 / 3}=0.0037 \mathrm{~s}\right)$, during the formation of a pseudo-plug. The four last profiles have been shifted by one wavelength to better guide the eye; (b) fully developed pseudo-plugs: $t^{\star} / t_{\nu}^{\star}=300$. The periodic surface profile has been reproduced three times here.

be alleviated by implementing a locally refined grid in the plug regions, following the example of Lister et al. (2006) for the representation of locally thin liquid films. We leave this task, and the confrontation of our pseudo-plug model with full-fledged plug models for pressure driven core-annular flows (Ubal et al. 2008) or inclined planar channel flows (Suresh \& Grotberg 2005), to future work. In the latter case, our additional force term (4.1) would have to be adapted to a planar geometry.

\section{Acknowledgements}

This work was supported by the ANR wavyFILM project, grant ANR-15-CE06-0016-01 of the French Agence Nationale de la Recherche. Declaration of Interests. The authors report no conflict of interest.

\section{REFERENCES}

Albert, C., Marschall, H. \& Bothe, D. 2013 Direct numerical simulation of interfacial mass transfer into falling films. International Journal of Heat and Mass Transfer 69, 343-357.

Alekseenko, S. V., Aktershev, S. P., Cherdantsev, A. V., Kharlamov, S. M. \& Markovich, D. M. 2009 Primary instabilities of liquid film flow sheared by turbulent gas stream. International Journal of Multiphase Flow 35, 617-627.

Aul, R. W. \& Olbricht, W. L. 1990 Stability of a thin annular film in pressure-driven, low-reynolds-number flow through a capillary. J. Fluid Mech. 215, 585-599.

Beltrame, P. 2018 Partial and complete wetting in a micro-tube. Europhysics Letters 121, 64002.

Benney, D. J. 1966 Long waves on liquid films. Journal of Mathematics and Physics 45, 150155.

Bian, S., Tai, C.-F., Halpern, D., Zheng, Y. \& Grotberg, J. B. 2010 Experimental study of flow fields in an airway closure model. Journal of Fluid Mechanics 647, 391-402. 
Brackbill, J. U., Kothe, D. B. \& Zemach, C. 1992 A continuum method for modelling surface tension. J. Comput. Phys. 100, 335-354.

Brooke Benjamin, T. 1957 Wave formation in laminar flow down an inclined plane. J. Fluid Mech. 2, 554-574.

Camassa, R., Forest, M. G., Lee, L., Ogrosky, H. R. \& Olander, J. 2012 Ring waves as a mass transport mechanism in air-driven core-annular flows. Phys. Rev. E 86 (6), 066305 .

Camassa, R., Marzuola, J. L, Ogrosky, H. R. \& Vaughn, N. 2016 Traveling waves for a model of gravity-driven film flows in cylindrical domains. Physica D 333, 254-265.

Camassa, R., Ogrosky, H. R. \& Olander, J. 2014 Viscous film-flow coating the interior of a vertical tube. part 1. gravity-driven flow. Journal of Fluid Mechanics 745, 682-715.

Camassa, R., Ogrosky, H. R. \& Olander, J. 2017 Viscous film-flow coating the interior of a vertical tube. part 2. air-driven flow. Journal of Fluid Mechanics 825, 1056-1090.

Chang, H. C., Demekhin, E. A. \& Kaldidin, E. 1996 a Simulation of noise-driven wave dynamics on a falling film. AIChE J. 42 (6), 1553-1568.

Chang, H. C., Demekhin, E. A., Kalaidin, E. \& Ye, Y. $1996 b$ Coarsening dynamics of falling-film solitary waves. Phys. Rev. E 54 (2), 1467-1477.

Chang, H. C., Demekhin, E. A. \& Kopelevich, D. I. 1993 Nonlinear evolution of waves on a vertically falling film. Journal of Fluid Mechanics 250, 433-480.

Dao, E. K. \& Balakotaiah, V. 2000 Experimental study of wave occlusion on falling films in a vertical pipe. AIChE J. 46 (7), 1300.

Delaunay, C. 1841 Sur la surface de révolution dont la courbure moyenne est constante. Journal de Mathémathiques Pures et Appliquées 6, 309-320.

Dietze, G. F. 2016 On the Kapitza instability and the generation of capillary waves. Journal of Fluid Mechanics 789, 368-401.

Dietze, G. F. 2019 Effect of wall corrugations on scalar transfer to a wavy falling liquid film. Journal of Fluid Mechanics 859, 1098-1128.

Dietze, G. F. \& Ruyer-Quil, C. 2013 Wavy liquid films in interaction with a confined laminar gas flow. J. Fluid Mech. 722, 348-393.

Dietze, G. F. \& Ruyer-Quil, C. 2015 Films in narrow tubes. Journal of Fluid Mechanics 762, 68-109.

Ding, Z., Liu, Z., Liu, R. \& YANG, C. 2019 Thermocapillary effect on the dynamics of liquid films coating the interior surface of a tube. International Journal of Heat and Mass Transfer 138, 524-533.

Doedel, E. J. 2008 AUTO07P: Continuation and bifurcation software for ordinary differential equations. Montreal Concordia University .

Duprat, C., Ruyer-Quil, C., Kalliadasis, S. \& Giorgiutti-Dauphiné, F. 2007 Absolute and convective instabilities of a viscous film flowing down a vertical fiber. Physical Review Letters 98, 244502.

Everett, D. H. \& Haynes, J. M. 1972 Model studies of capillary condensation. J. Colloid Interface Sci. 38 (1), 125-137.

Frenkel, A. L., Babchin, A. J., Levich, B. G., Shlang, T. \& Sivashinsky, G. I. 1987 Annular flows can keep unstable films from breakup: nonlinear saturation of capillary instability. J. Colloid Interface Sci. 115 (1), 225-233.

Gauglitz 1988 An extended evolution equation for liquid film breakup in cylindrical capillaries. Chem. Eng. Sci. 43 (7).

Goren, S. L. 1962 The instability of an annular thread of fluid. J. Fluid Mech. 12 (2), 309-319.

Grotberg, J. 2011 Respiratory fluid mechanics. Phys. Fluids 23, 021301.

Hickox, C. E. 1971 Instability due to viscosity and density stratification in axisymmetric pipe flow. Phys. Fluids 14 (2), 251-262.

Hirt, C. W. \& Nichols, B. D. 1981 Volume of fluid (VOF) method for the dynamics of free boundaries. J. Comput. Phys. 39, 201-225.

Jensen, O. E. 2000 Draining collars and lenses in liquid-lined vertical tubes. J. Colloid Interface Sci. 221, 38-49.

Joseph, D. D., Chen, K. P. \& Renardy, Y. Y. 1997 Core-annular flows. Annu. Rev. Fluid Mech. 29, 65-90. 
Kalliadasis, S. \& Chang, H. C. 1994 Drop formation during coating of vertical fibres. Journal of Fluid Mechanics 261, 135-168.

Kalliadasis, S., Ruyer-Quil, C., Scheid, B. \& Velarde, M. G. 2012 Falling Liquid Films, Applied Mathematical Sciences, vol. 176. Springer Verlag.

Kamm, R. D. \& Schroter, R. C. 1989 Is airway closure caused by a liquid film instability? Respiration Physiology 75, 141-156.

Kapitza, P. L. 1948 Wave flow of thin layer of viscous fluid (in Russian). Zhurn. Eksper. Teor. Fiz. 18 (1), 3-28.

King, M. \& Macklem, P. T. 1977 Rheological properties of microliter quantities of normal mucus. Journal of Applied Physiology 42 (6), 797-802.

Kouris, C. \& Tsamopoulos, J. 2001 Dynamics of axisymmetric core-annular flow in a straight tube. I. the more viscous fluid in the core, bamboo waves. Phys. Fluids 13 (4), 841-858.

Lavalle, G., Grenier, N., Mergui, S. \& Dietze, G. F. to be published Solitary waves on superconfined falling liquid films. Physical Review Fluids .

Lewis, T. A., Tzeng, Y.-S.and McKinstry, E. L., Tooker, A. C., Hong, K., Sun, Y., Mansour, J., Handler, Z. \& Albert, M. S. 2005 Quantification of airway diameters and 3d airway tree rendering from dynamic hyperpolarized $3 \mathrm{He}$ magnetic resonance imaging. Magnetic Resonance in Medicine 53, 474-478.

Lister, J .R., Rallison, J. M., King, A. A., Cummings, L. J. \& Jensen, O. E. 2006 Capillary drainage of an annular film: the dynamics of collars and lobes. J. Fluid Mech. 552, 311-343.

Liu, J. \& Gollub, J. P. 1993 Onset of spatially chaotic waves on flowing films. Physical Review Letters 70 (15), 2289-2292.

Liu, R. \& Ding, Z. 2017 Stability of viscous film flow coating the interior of a vertical tube with a porous wall. Physical Review E 95 (5), 053101.

Mathematica 2014 Version 10.0.2.0. Wolfram Research, Inc., Champaign, IL.

Nosoko, T., Yoshimura, P. N., Nagata, T. \& Oyakawa, K. 1996 Characteristics of twodimensional waves on a falling liquid film. Chemical Engineering Science 51 (5), 725-732.

Plateau, J. P. 1849 Recherches expérimentales et théorique sur les figures d'équilibre d'une masse liquide sans pesanteur. Mémoires de l'Académie Royale des Sciences, des Lettres et des Beaux-Arts de Belgique 23, 1-1.

Popinet, S. 2009 An accurate adaptive solver for surface-tension-driven interfacial flows. $J$. Comput. Phys. 228, 5838-5866.

QuÉRÉ, D. 1990 Thin films flowing on vertical fibers. Europhysics Letters 13 (8), 721-726.

Quéré, D. 1999 Fluid coating on a fibre. Annu. Rev. Fluid Mech. 31, 347-384.

RAYleigh, LORD 1892 On the instability of cylindrical fluid surfaces. Phylosophical Magazine 34 (207), 177-180.

Richard, G., Ruyer-Quil, C. \& Vila, J. P. 2016 A three-equation model for thin films down an inclined plane. Journal of Fluid Mechanics 804, 162-200.

Seebauer, F., Poechlauer, P., Braune, S. \& Steinhofer, S. 2012 Tube bundle falling film microreactor for performing gas liquid reactions. US Patent 8221708B2.

ShkAdov, V. YA. 1967 Wave flow regimes of a thin layer of viscous fluid subject to gravity. Fluid Dyn. 2 (1), 29-34.

Suresh, V. \& Grotberg, J. B. 2005 The effect of gravity on liquid plug propagation in a two-dimensional channel. Physics of Fluids 17 (3), 031507, arXiv: https://doi.org/10.1063/1.1863853.

Thiele, U., Velarde, M. G., Neuffer, K \& Pomeau, Y. 2001 Sliding drops in the diffuse interface model coupled to hydrodynamics. Physical Review E 64 (6), 061601.

TRIFonov, Y. Y. 1992 Steady-state traveling waves on the surface of a viscous liquid film falling down on vertical wires and tubes. AIChE J. 38 (6), 821-834.

Trifonov, Y. Y. 2010 Counter-current gas-liquid wavy film flow between the vertical plates analyzed using the Navier-Stokes equations. AIChE Journal 56 (8), 1975-1987.

Tseluiko, D. \& Kalliadasis, S. 2011 Nonlinear waves in counter-current gas-liquid film flow. J. Fluid Mech. 673, 19-59.

Ubal, S., Campana, D. M., Giavedoni, M. D. \& Saita, F. A. 2008 Stability of the steadystate displacement of a liquid plug driven by a constant pressure difference along a prewetted capillary tube. Industrial \& Engineering Chemistry Research 47, 6307-6315. 
Vlachos, N. A., Paras, S. V., Mouza, A. A. \& Karabelas, A. J. 2001 Visual observations of flooding in narrow rectangular channels. Int. J. Multiphase Flow 27, 1415-1430.

Wray, A. W. 2013 Electrostatically controlled large-amplitude, non-axisymetric waves in thin film flows down a cylinder. J. Fluid Mech. 736, R2.

Xu, F. \& Jensen, O. 2017 Trapping and displacement of liquid collars and plugs in rough-walled tubes. Physical Review Fluids 2, 094004.

Yıн, C. S. 1963 Stability of liquid flow down an inclined plane. The Physics of Fluids 6 (3), 321-334.

Yoshimura, P. N., Nosoko, P. \& Nagata, T. 1996 Enhancement of mass transfer into a falling laminar liquid film by two-dimensional surface waves-some experimental observations and modeling. Chemical Engineering Science 51 (8), 1231-1240.

ZApke, A. \& KröGer, D. G. 2000 Countercurrent gas-liquid flow in inclined and vertical ducts - I: Flow patterns, pressure drop characteristics and flooding. International Journal of Multiphase Flow 26, 1439-1455.

Zhou, Z.-Q., Peng, J. \& Zhang, Y.-J. Zhuge, W.-L. 2016 Viscoelastic liquid film flowing down a flexible tube. Journal of Fluid Mechanics 802, 583-610. 